\title{
Modified Morris-Lecar neuron model: Effects of very low frequency electric fields and of magnetic fields on the local and network dynamics of an excitable media
}

\section{Karthikeyan Rajagopal}

Chennai Institute of Technology

Irene Moroz

Oxford University: University of Oxford

\section{Balamurali Ramakrishnan}

Chennai Institute of Technology

Anitha Karthikeyan ( $\sim$ anitha.karthikeyan@tdtu.edu.vn )

Ton Duc Thang University https://orcid.org/0000-0001-6485-4687

\section{Prakash Duraisamy}

Chennai Institute of Technology

\section{Research Article}

Keywords: Neuron field effects, bifurcation, spiral waves, noise

Posted Date: February 25th, 2021

DOI: https://doi.org/10.21203/rs.3.rs-170503/v1

License: (c) (1) This work is licensed under a Creative Commons Attribution 4.0 International License.

Read Full License 


\title{
Modified Morris-Lecar neuron model: Effects of very low frequency electric fields and of magnetic fields on the local and network dynamics of an excitable media
}

\author{
Karthikeyan Rajagopal ${ }^{a}$, Irene Moroz ${ }^{b}$, Balamurali Ramakrishnan ${ }^{c}$, \\ Anitha Karthikeyn ${ }^{\text {, Prakash Duraisamy }}{ }^{e}$ \\ ${ }^{a, c, e}$ Center for Nonlinear Systems, Chennai Institute of Technology, India. \\ akarthikeyan.rajagopal@,citchennai.net \\ abalamurali@citchennai.net \\ fprakash.duraisamy@citchennai.net \\ ${ }^{b}$ Mathematical Institute, University of Oxford, Andrew Wiles Building, Oxford, UK. \\ irene.Moroz@maths.ox.ac.uk \\ $d^{*}$ Nonlinear Systems and Applications, Faculty of Electrical and Electronics Engineering, Ton \\ Duc Thang University, Ho Chi Minh City, Vietnam. \\ anitha.karthikeyan@tdtu.edu.vn \\ *Corresponding author
}

\begin{abstract}
A Morris-Lecar neuron model is considered with Electric and Magnetic field effects where the electric field is a time varying sinusoid and magnetic field is simulated using an exponential flux memristor. We have shown that the exposure to electric and magnetic fields have significant effects on the neurons and have exhibited complex oscillations. The neurons exhibit a frequencylocked state for the periodic electric field and different ratios of frequency locked states with respect to the electric field frequency is also presented. To show the impact of the electric and magnetic fields on network of neurons, we have constructed different types of network and have shown the network wave propagation phenomenon. Interestingly the nodes exposed to both electric and magnetic fields exhibit more stable spiral waves compared to the nodes exhibited only to the magnetic fields. Also, when the number of layers are increased the range of electric field frequency for which the layers exhibit spiral waves also increase. Finally the noise effects on the field affected neuron network are discussed and multilayer networks supress spiral waves for a very low noise variance compared against the single layer network.
\end{abstract}

Keywords: Neuron field effects; bifurcation; spiral waves; noise;

\section{Introduction}

Neurons can generate, transmit, receive signals to and from brains by firing several types of electrical activities. Based on the firing patterns and frequency-current curves, the excitability of neurons can be classified [1] with classes 1,2 and 3 which is more valuable for investigating behavior of neuronal system under different scenario. Class 3 neuron do not show any spiking 
behavior, whereas class $1 \& 2$ exhibit firing frequency and literatures [2] proved Morris-Lecar (M-L) model is a fruitful test-ground for bifurcation analysis of these types. The repetitive firing fashion of such neurons leads to saddle node bifurcation into subcritical Hopf bifurcation. The firing property is much influenced with stimulating current for a particular range, M-L model exposes Bogdanov-Takens bifurcation. In [3] different membrane excitability is introduced into M-L model and revealed the bistability property. While the coupling strength increased, more limit cycles are formed beyond the Hopf bifurcations which directly affect the complexity of the system. The coupling strength plays a vital role on bifurcation analysis as well as in synchronization stability. The neurophysiological studies in neuron network discloses some intricate behaviours such as spiking, bursting, transition of modes, extreme multistability, synchronous transition. On the other hand, network patterns can be portrayed with considering electromagnetic radiation on conventional neuron models.

Based on the electromagnetic field theory, the significant effect of electromagnetic radiation can be defined with magnetic flux. The coupling between magnetic flux and membrane potential influences network behaviour. With these considerations, memristor based models were generated [4-6]. The effect of electromagnetic induction on synchronization, spatiotemporal behaviour of coupled neurons and neuron networks is extensively studied in [7-11]. The appearance of different spatiotemporal patterns confirms the complexity in dynamical behaviour of the system. Spatiotemporal patterns can coordinate oscillation phases in neuronal network arrays and identified potentials to bind sensory information over a population of neurons. Spiral waves are an elegant pattern observed in many biological systems, experiments with voltagesensitive dye imaging exposed the formulation of spiral waves in neocortical slices $[12,13]$. Hence, investigating the formation and growth of spiral waves becomes a fertile field in network dynamics. Meanwhile the need for control scheme to suppress such patterns also increased. Some effective control algorithms are developed and discussed in [14-19], most of them deals with the excitation media. The studies demonstrate how chaotic spatiotemporal behaviours appear for different levels of electromagnetic field. The external electromagnetic field boosts mean firing rate on the neuron network and interrupts the synchronization scheme. Spatiotemporal patterns can be suppressed to a homogeneous resting state with altering the exposed electromagnetic field. Higher values of magnetic flux results with decrement in duration and width of the oscillatory pattern. However chaotic pattern and propagation fashion are so sensitivity to the initial conditions.

From the above discussion, the research spectrum is narrow down to M-L model influenced with electromagnetic effect to study the synchronization and network behaviours of neuronal systems. Experimental studies conducted on humans and animals exposed to two types of electromagnetic field (i) Extremely low frequency electromagnetic field (ELF-EF) generally produced by electrical and electronic gadgets (ii) Radio frequency magnetic fields (RF-MF) produced by wireless devices such as mobile and transmission towers. Low frequency timevarying magnetic field induced currents can alter neuroprocessing in the central nervous system [12]. However, the induced currents are very weak, networks of neurons are more sensitive to weak fields compared with isolated neurons. In this paper we considered a modified Morris-Lecar (ML) neuron model supplied with low frequency electromagnetic field, and investigate dynamic 
behaviours particularly network dynamics and wave propagation in multi-layer neuron topology. The Box-Muller type noise are considered to suppress spiral waves.

\section{The modified Morris-Lecar neuron model}

Using the same notation as in [20], the modified Morris-Lecar Neuron model whose electrical equivalent is shown in Fig.1, incorporating an electric and magnetic field effects can be derived as,

$$
\begin{aligned}
C \dot{V} & =G_{c a} M_{\infty}\left(V_{c a}-V-V_{e}\right)+G_{k} W\left(V_{k}-V-V_{e}\right) \\
& +G_{L}\left(V_{L}-V-V_{e}\right)-I-k_{0} \rho(\phi) V \\
\dot{W} & =\tau_{\infty}\left(W_{\infty}-W\right) \\
\dot{I} & =\varepsilon\left(V_{0}+V\right) \\
\dot{\phi} & =k_{1} V-k_{2} \phi
\end{aligned}
$$

where $V_{e}$ is the external electric field effect and the parameters required for the simulation are used from [20] and the electric field is $V_{e}=E \sin (2 \pi \omega t)$, The other functions are defined as

$$
\begin{aligned}
M_{\infty} & =0.5\left(1+\tanh \left(\frac{V-V_{1}}{V_{2}}\right)\right) ; W_{\infty}=0.5\left(1+\tanh \left(\frac{V-V_{3}}{V_{4}}\right)\right) \\
\tau_{\infty} & =\frac{1}{\sigma} \cosh \left(\frac{V-V_{3}}{2 V_{4}}\right) ; \rho(\phi)=\alpha+3 \beta^{2}
\end{aligned}
$$

The fixed points of equ (1) are obtained by setting the time derivatives to zero, so that

$$
\begin{aligned}
V & =-V_{0} ; W=W_{\infty} ; \phi=\frac{-k_{1} V_{0}}{k_{2}} \\
I= & G_{c a} M_{\infty}\left(V_{c a}-V-V_{e}\right)+G_{\kappa}\left(V_{k}-V-V_{e}\right) \\
& +G_{L}\left(V_{L}-V-V_{e}\right)-k_{0} \rho(\phi) V_{e}
\end{aligned}
$$

There is therefore only one fixed point, which is obtained by substituting (3a) into (3b). Its linear stability is then found by computing the fourth order Jacobian matrix: associated with the RHS of (1). This procedure was described in some detail in [20]. Here our objectives are different. We are interested in the consequences of modifying the potential $V$ with a low frequency periodic oscillation. 


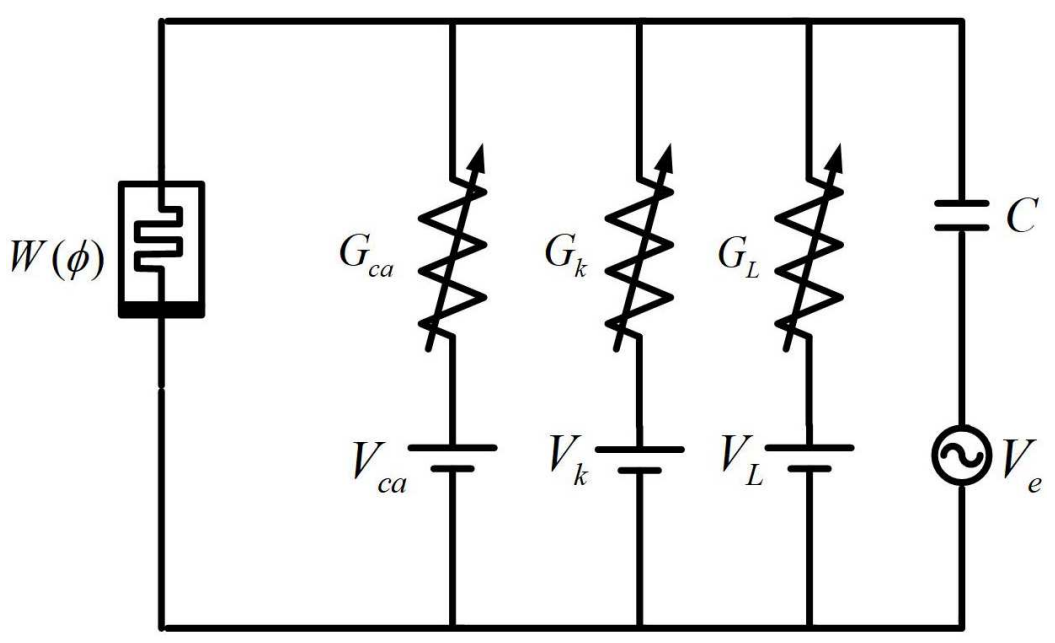

Fig, 1: The circuit model of the M-L neuron exposed to electric and magnetic fields

\subsection{Constant applied electric field}

Following [30], we firstly consider the case of a constant electric field of small amplitude, by taking $V_{e}=0.1$ to be a constant. If we then plot a bifurcation transition diagram of $V_{\max }$ as $k_{0}$ increases, we lose all the chaotic states, shown in Fig 4a of [20]. The following figure shows the outcome:

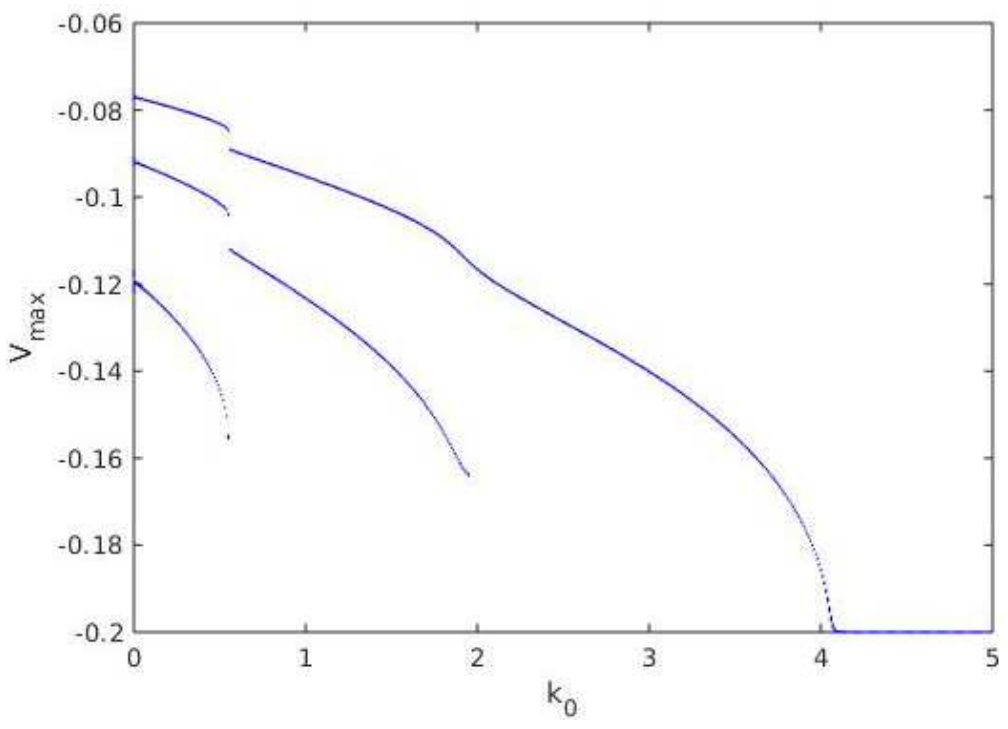

Fig 2: Bifurcation transition plot of $V_{\max }$ as $k_{0}$ increases when $V_{e}$ is a constant with $E=0.1$

There is still a supercritical Hopf bifurcation, but for a different value for $k_{0}$ (for $k_{0} \approx 4.2$ ). When we repeat the numerical analysis for $E=0.5$, the system evolves to a steady state:

$(V, W, I, \phi)=(-0.2,0.61441759 E-05,-0.91246803 E-01,-0.04)$ We now replace $V_{e}$ by a sinusoidally varying electric field.

\subsection{Externally applied sinusoidally electric field}


There are two parameters of interest in this system: the frequency $\omega$ of the electrical forcing and its amplitude $E$. For very low frequency field (ELF) we take $0 \leq E \leq 5$ and $0 \leq \omega \leq 10$.

Figure 3 shows a bifurcation transition diagram as the frequency $\omega$ increases in terms of $V_{\max }$ for fixed amplitude $E=0.1$. The top panel shows part of the diagram for $0 \leq \omega \leq 1$. For $\omega>1$ (not shown here) we found mainly chaotic behaviour apart from a few isolated windows of periodic states e.g., at $\omega=2.265, \omega=2.405$ and $\omega=2.435$. The lower panel of figure 2 shows an enlargement of the region between $0 \leq \omega \leq 0.3$.

There are a number of periodic windows in figure 3, which represent frequency locked states. Figure 4 shows a selection of six examples of $V$ from Figure 3b, together with the corresponding forcing function $V_{e}$ for $0.03 \leq \omega \leq 0.185$. We show examples of (a) a 3:2-locked state for $\omega=0.145$ and (f) a 10:8-locked state for $\omega=0.185$.

If we increase $E$ to $E=0.5$, we obtain the bifurcation transition diagram shown in figure 5. The regions of chaotic behaviour for $\omega<1$ have been replaced by periodic windows, apart from a small
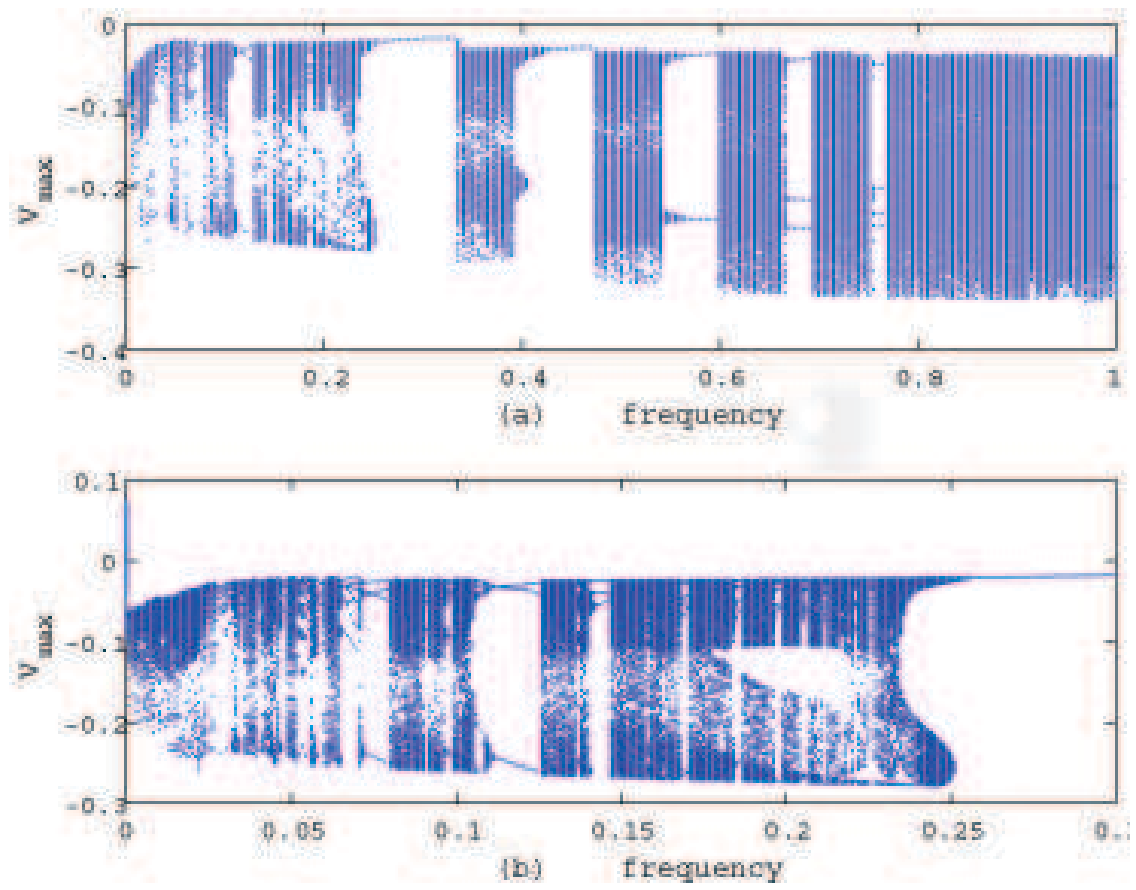

Fig 3: Bifurcation transition plot of $V_{\max }$ as frequency $\omega$ increases for forcing amplitude $E=0.1$.

The top panel (a) shows the behaviour for $0 \leq \omega \leq 1$, while the lower panel (b) shows an enlargement of the region $0 \leq \omega \leq 0.3$

region between $0.3 \leq \omega \leq 0.4$. When $E=1$, the upper panel of figure 6 shows the behaviour for $0 \leq \omega \leq 5$, while the lower panel shows a blow-up of the region for $0 \leq \omega \leq 0.8$.

Figure 6 shows the corresponding scenario for $E=1$. The upper panel shows the range from $0 \leq \omega \leq 5$. There is a large region of periodic states for $0 \leq \omega \leq 3.165$. The middle panel show part of this region for $0 \leq \omega \leq 0.6$. The lower panel shows the range $3 \leq \omega \leq 4$. After the period- 3 and 
period-4 cycles, there is a region of quasi-periodic states for $3.165 \leq \omega \leq 3.9$. Figure 8 shows an example of a 16:1
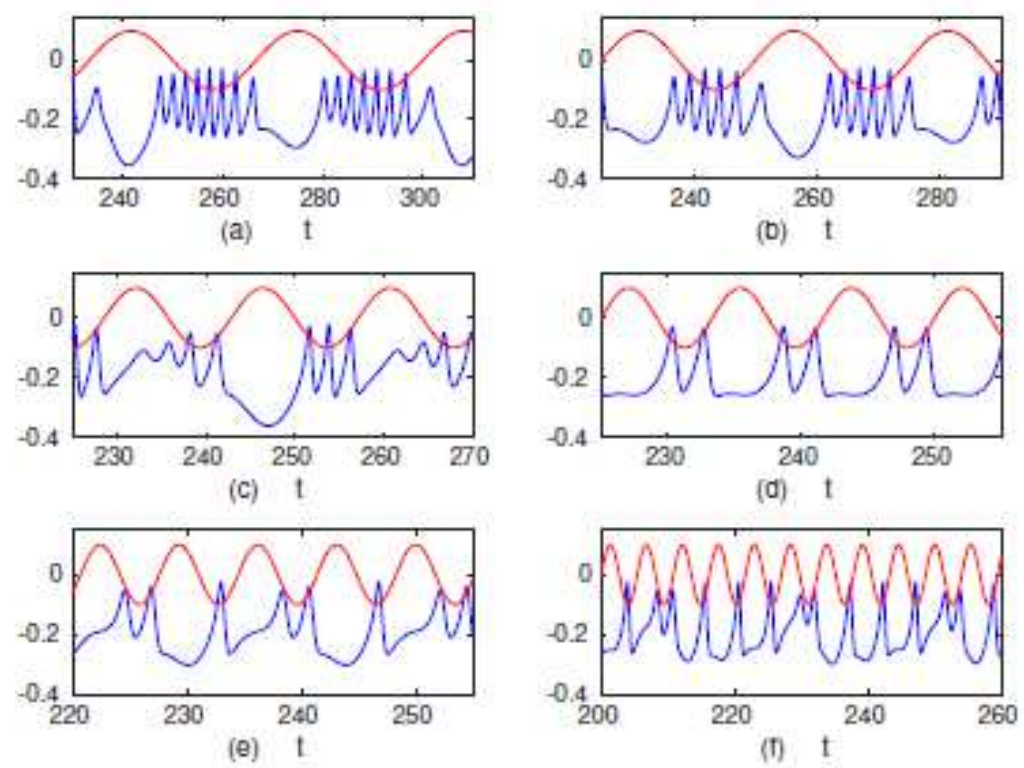

Figure 4: A selection of six frequency-locked states for $V$ (blue curves) for forcing amplitude $E=0.1$, together with the forcing function $V_{e}$ (red curves). (a) $\omega=0.03$ a 16:2 frequency locked state; (b) $\omega=0.04$ a 12:2 locked state; (c) $\omega=0.07$ an 8:2 locked state; (d) $\omega=0.12$ a 2:1 locked state; (e ) $\omega=0.145$ a 3:2 locked state; (f) $\omega=0.185$ a 10:8 locked state.

Frequency-locked state for $\omega=0.05$ and $E=1$. Again, the forcing function is shown in red and the time series for $V$ is shown in blue. Figure 8 shows an example of a quasi-periodic state for $\omega=3.5$. The upper panel show the time series for $V(t)$, while the lower panel shows the forcing term. Figure 9 shows a selection of phase portraits for $\omega=3.5$. Figures 9(a)-(c) show evidence of the quasi-periodicity, while the projection into the $(I, \phi)$-plane appears periodic. Finally figure 10 shows an example of a chaotic state for $\omega=4$. 


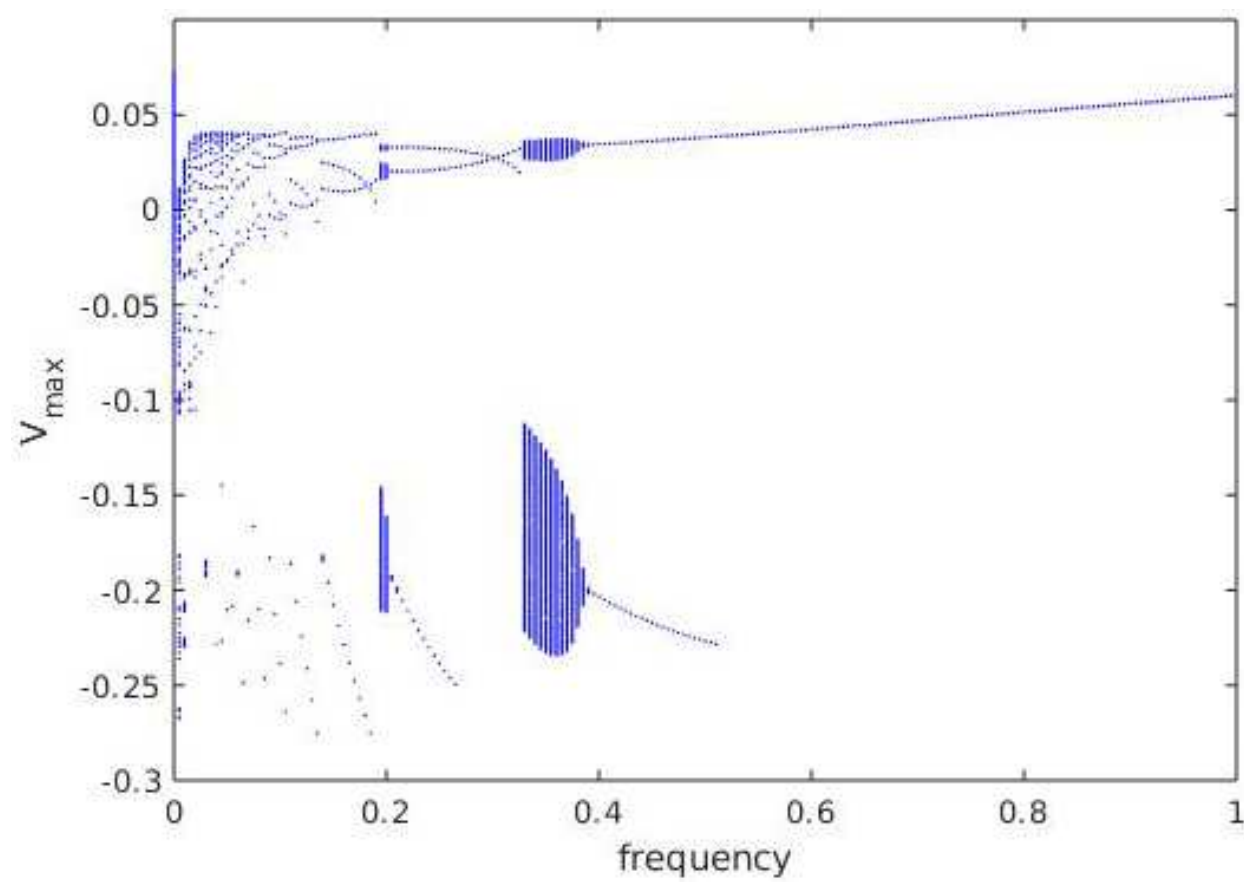

Fig 5: Bifurcation transition plot of $V_{\max }$ as frequency $\omega$ increases for forcing amplitude $E=0.5$. The chaotic dynamics for $\omega<0.3$ has been replaced by frequency-locked behaviours.
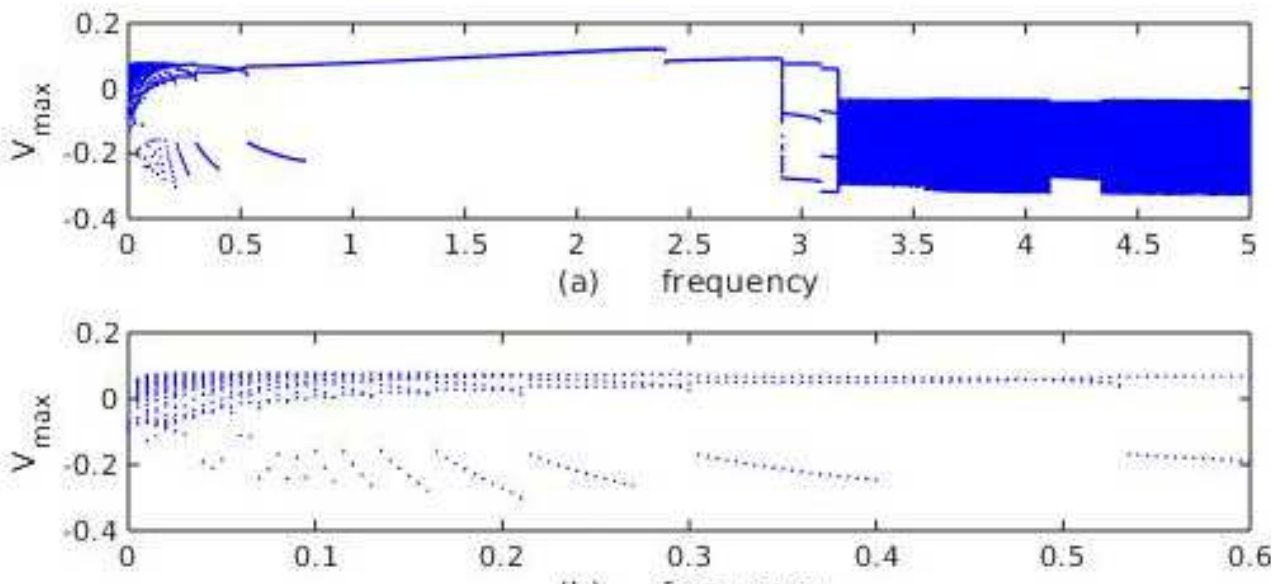

(b) frequency

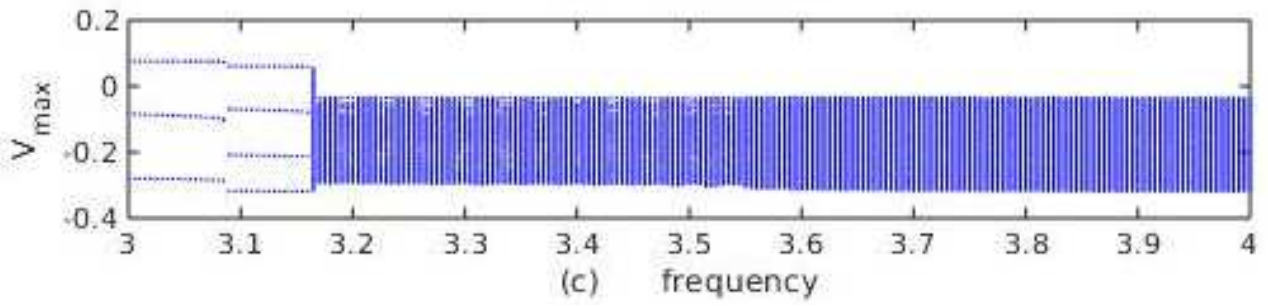

Fig 6: Bifurcation transition plot of $V_{\max }$ as frequency $\omega$ increases for forcing amplitude $E=1$. (a) for $0 \leq \omega \leq 5$; (b) a section for $0 \leq \omega \leq 0.8$; (c) a section for $3 \leq \omega \leq 4$. 


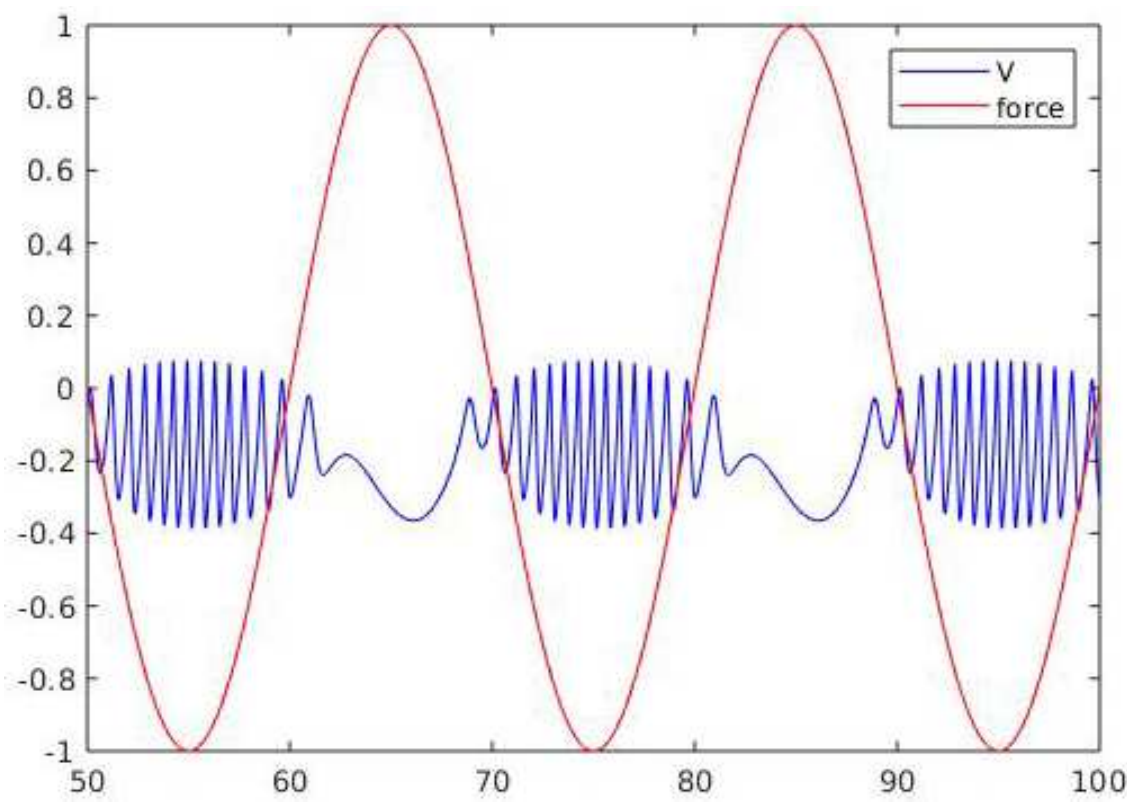

Fig 7: A 16:1 frequency-locked state for $E=1$ and $\omega=0.05$

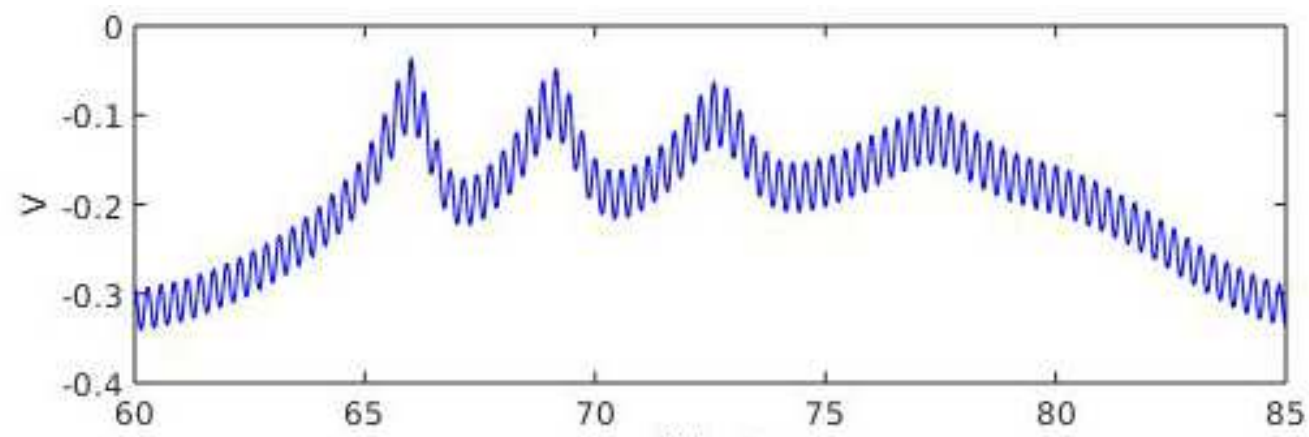

(a) $\mathrm{t}$

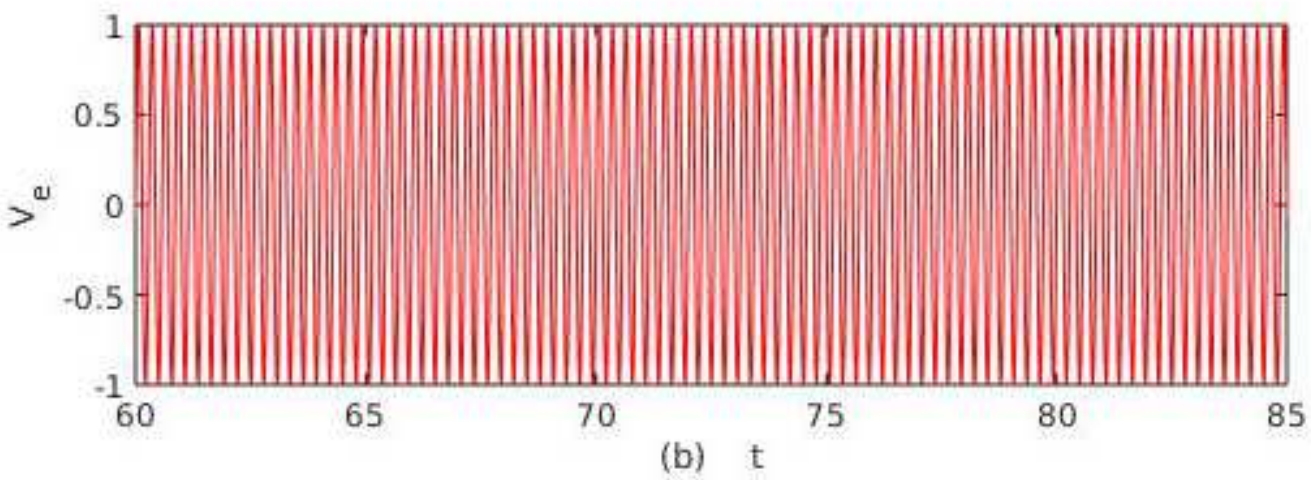

Fig 8: A section of the time series for $V$, showing a quasi-periodic state for $E=1$ and frequency $\omega=3.5$. The upper panel shows $V$, while the lower panel shows the forcing frequency. 

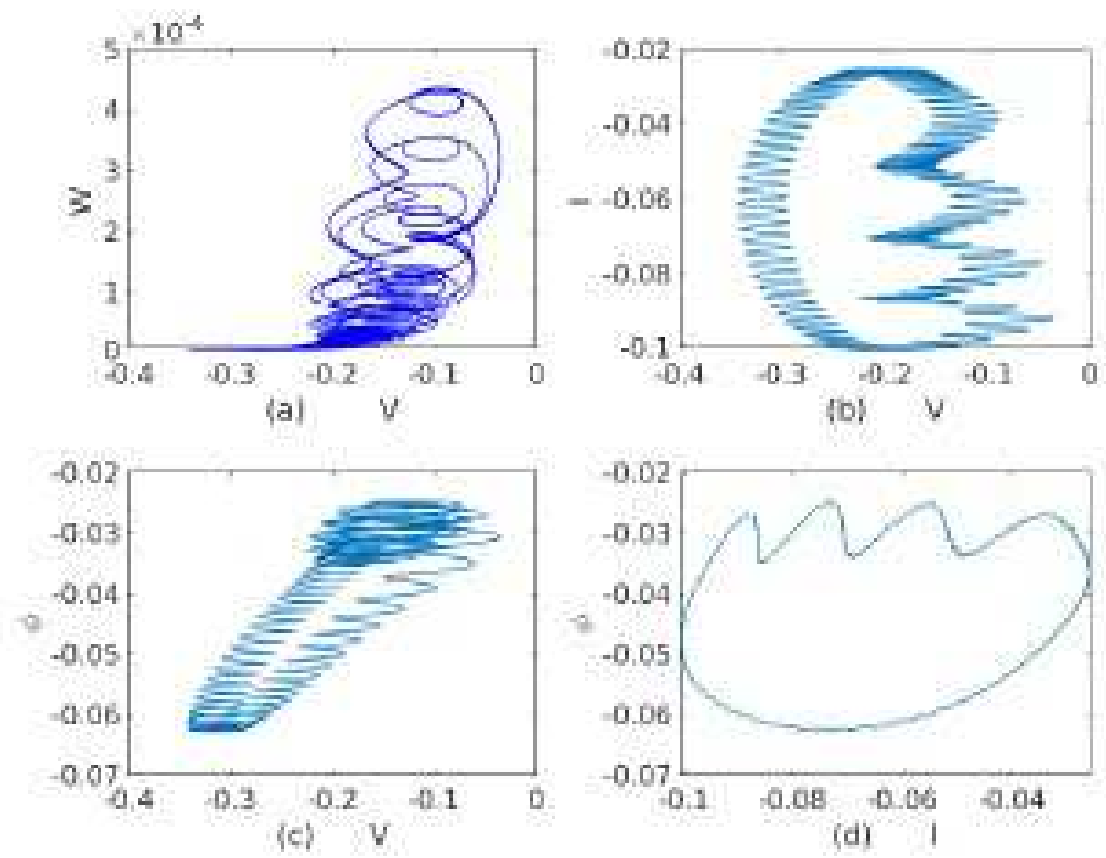

Fig 9: Phase portraits for the quasi-periodic state for $E=1$ and frequency $\omega=3.5$.
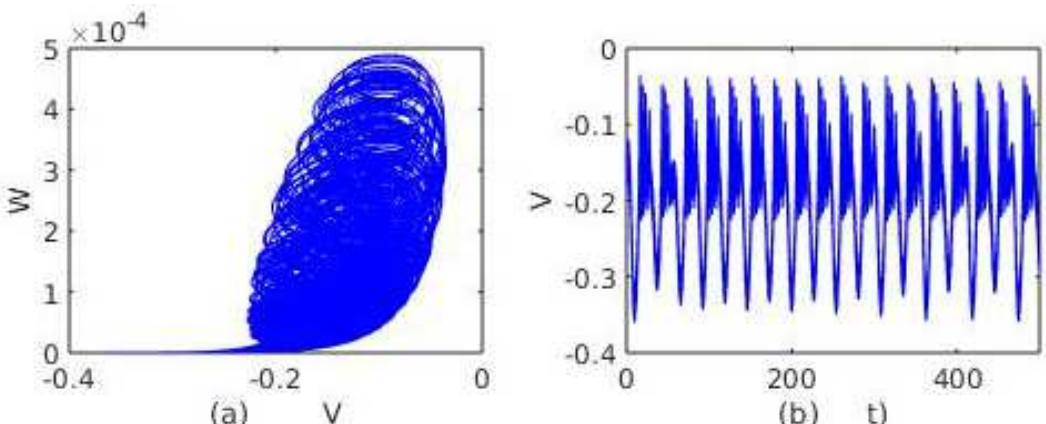

(a)

(b) t)
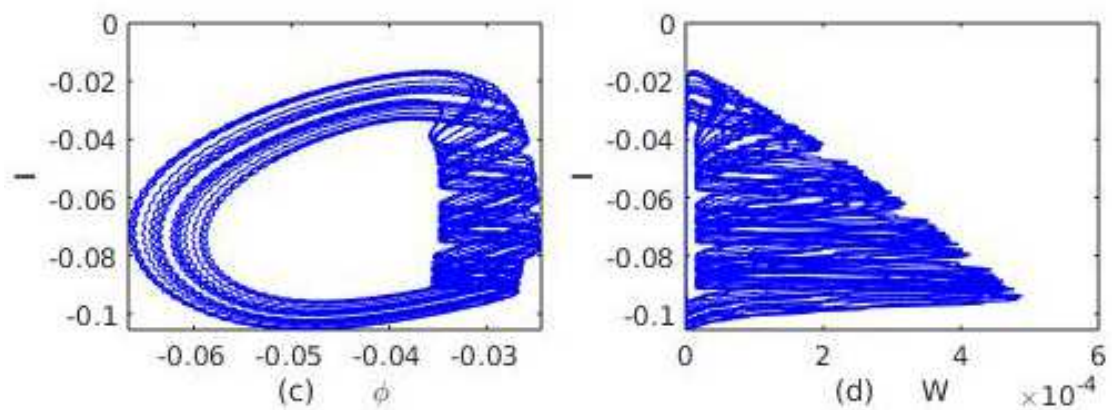

Fig 10: Phase portraits for the chaotic state for $E=1$ and frequency $\omega=4$.

\section{Wave propagation in the ML-EM network:}

To completely investigate the network dynamics of the ML-EM network, we constructed lattice arrays with single and multiple layers. To be more specific, we investigated the wave propagation phenomenon in single, two- and three-layer networks. Under each network, we investigate two cases with first case considering only electric field effects and second case considering both 
electric and magnetic fields. The stimuli force applied to the network is considered periodic $(F(t)=A \sin (\Omega t))$ with amplitude $A=0.1$ and frequency $\Omega=0.01$ fixed for all the investigations. Further the network is considered to have no flux boundaries and the stimuli force is applied to the network from the left boundary. The ML-EM system parameters are considered as in [1] with only the electrical field frequency and amplitude considered as control parameters. For all three network types we have used the RK4 method for the numerical analysis with step size $\mathrm{h}=0.01$ and simulation time of $5000 \mathrm{~s}$.

\subsection{Network performance in a single layer network:}

The first scenario of investigation is the two-dimensional lattice array of ML-EM neurons as shown in (4).

$$
\begin{aligned}
& C \dot{V}_{i j}=G_{C a} C a_{\infty}\left(V_{C a}-V_{i j}-V_{e}\right)+G_{K} \theta_{i j}\left(V_{K}-V_{i j}-V_{e}\right) \\
& +G_{L}\left(V_{L}-V_{i j}-V_{e}\right)-S_{i j}-k_{0} W\left(\phi_{i j}\right) V_{i j}+C\left(V_{i j}\right) \\
& +F(t) \beta_{i \theta_{1}} \beta_{j \theta_{2}} \\
& \dot{\theta}_{i j}=\tau_{\theta}\left(K_{\infty}-\theta_{i j}\right) \\
& \dot{S}_{i j}=\mu\left(V_{0}+V_{i j}\right) \\
& \dot{\phi}_{i j}=k_{1} V_{i j}-k_{2} \phi_{i j}
\end{aligned}
$$

The stimulus $F(t)$ is applied to the network in the left boundary using the conditions $\beta_{i \theta_{1}}=$ $1, \beta_{j \theta_{2}}=1$ for $[i, j]=[110,0]$. The nearest neighbour coupling function is defined as $C\left(V_{i j}\right)=$ $\sigma\left(V_{i+1 j}+V_{i-1 j}+V_{i j+1}+V_{i j-1}-4 V_{i j}\right)$ with $\sigma$ being the diffusion parameter and in this case we keep it as $\sigma=1$. In this section we will investigate two cases wherein in the first case we consider that the neurons are exposed to only electric field effect which minimises the equation (1) to first three states with $k_{0}=0$. In the second case we consider that the neuron is exposed to both electric and magnetic fields for which all four state variables are considered with $k_{0}=0.1$. As the main aim of this research is to study the electric field effects on the ML neuron model, we consider the frequency $(\omega)$ of the periodic electric field $V_{e}=E \sin (2 \pi \omega t)$ as the control parameter.

In Fig. 11 we investigate the wave propagation in the lattice network (4) considering the exposure of the nodes to only the periodic electric field. As defined, the stimuli force is applied to the left boundary of the network for a brief period of 100s-1000s. For the values of the frequency $\omega<$ 0.95 , all the nodes in the network goes unstable and the wave propagation was not supported. When the frequency of the electric field is increased to $\omega=0.95$, most of the nodes in the network starts oscillations except few nodes which are idle. This is because of these nodes been driven out of the spiking/bursting regions of initial conditions due to the applied stimuli force. Such situations are often seen due to the sharp change in the conductance of sodium ions in the neurons [21]. By increasing the frequency of electric field to $\omega=0.98$, all the nodes in the network are now in oscillation mode and thus we could show the multiarmed spiral waves with smaller radius of rotation occupying the entire network. Such spiral waves are seen in the range $0.98 \leq \omega \leq 1$ but when the frequency is increased to $\omega=1.05$, some of these spiral waves start breaking into multiple smaller spiral seeds while those with longer arms combine together to form spiral waves with very less frequency of rotation and larger radii. For $\omega=1.1$ the smaller radii spiral seeds are destroyed while those with longer arms diminishes and by increasing the frequency to $\omega=1.2$ 
they get destroyed and thus the spiral waves are no longer seen in the network. Thus the effective spiral wave range is $0.98 \leq \omega \leq 1.1$.
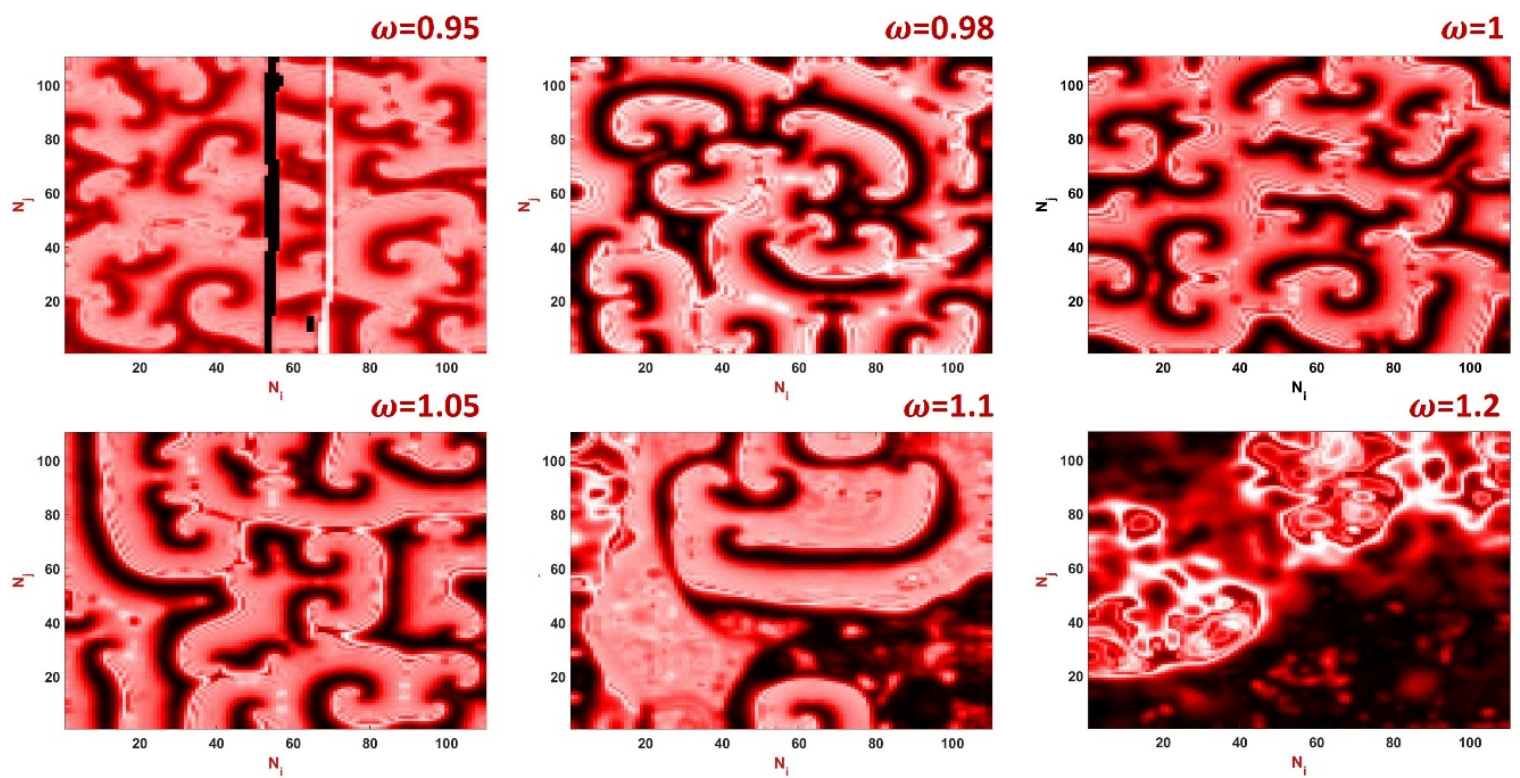

Fig.11: Wave propagation in the network (4) considering the ML-EM neurons with electric field for different values of $\omega$

In the second case of this investigation, we consider that the neurons are exposed to both electric and magnetic fields. The magnetic field coupling strength $k_{0}=0.1$ and the stimuli is applied to the left boundary of the network. Fig. 12 shows the captured wave propagation snap shots of the network for various electric field excitation frequency. It is clear that the spiral waves are seen for a longer range of frequency compared to the network discussed without magnetic field (Fig.11). This is because of the magnetic field coupling driving all the neurons in to spiking/bursting type oscillations and hence there are no idle nodes in the network which was not the case in Fig.11. For $\omega=0.9$, the network shows spiral waves and has multiarmed spiral seeds seen in the entire network. By increasing the frequency these small spiral seeds merge together to form long arm spiral waves for $\omega=1.1$ with only few centre of rotation. Further increasing the frequency to $\omega=$ 1.2 the spiral waves align towards the four corners of the network but soon starts dissipating from the right boundary as we increase the frequency to $\omega=1.3$. This is because of the nodes entering idle states and these idle states spread to all the nodes when the frequency is increased to $\omega=1.4$. Thus we could confirm that the spiral waves are seen for a much longer range of frequency $0.9 \leq$ $\omega<1.4$ against the spiral wave range for network exposed to electric field alone (Fig.10) $0.98 \leq$ $\omega \leq 1$. Thus magnetic field can be easily used to control the spiral waves in the network by either inducing or suppressing such turbulent spiral waves. 

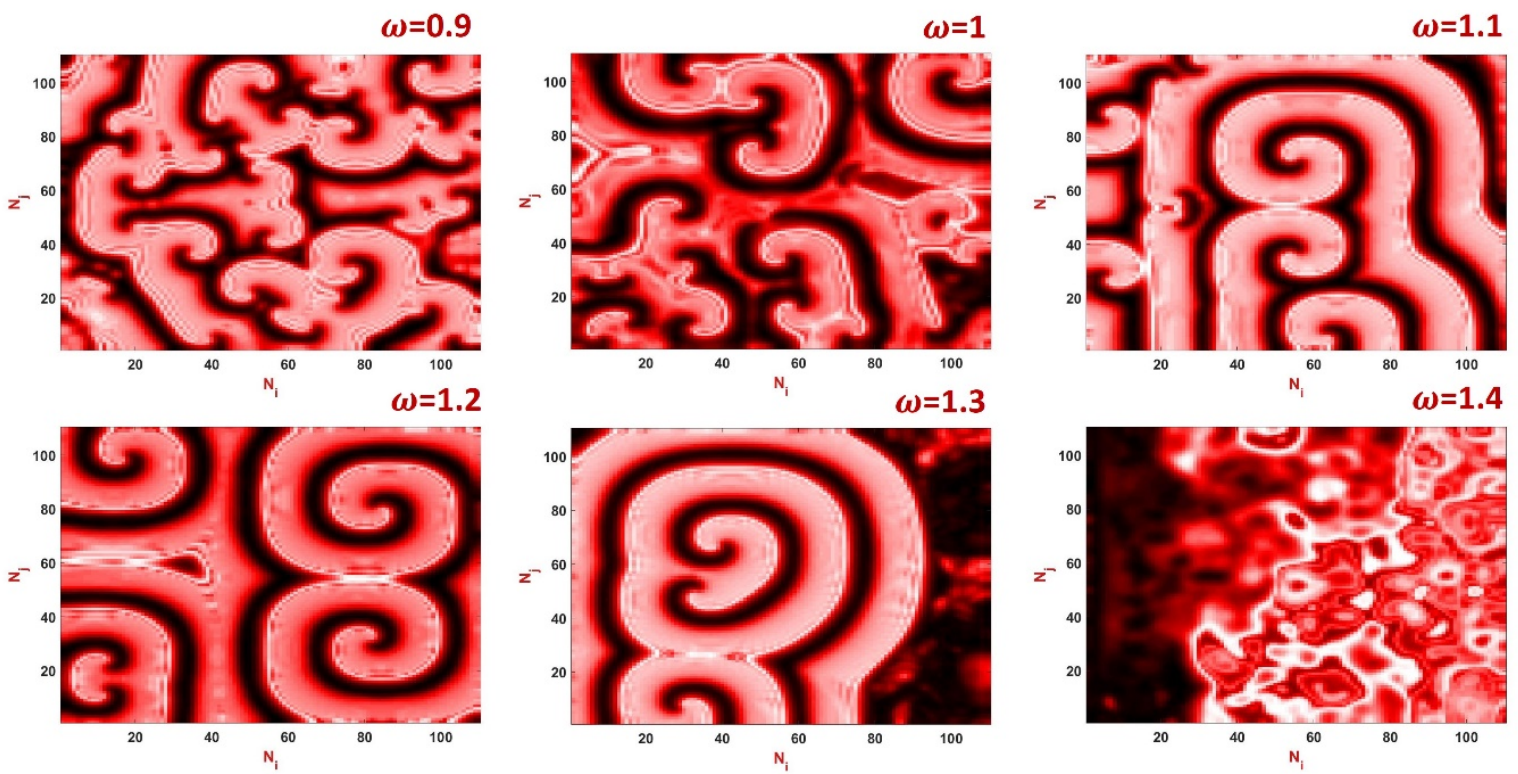

Fig.12: Wave propagation in the network (10) considering the ML-EM neurons with electric and magnetic fields for different values of $\omega$

\subsection{Network performance in a two-layer network:}

The second investigation is on the two-layer network with each layer constructed using the lattice array of ML-EM neurons. The mathematical model of the two-layer network is given in (11) where we considered a simple inter layer coupling as discussed in [22-24].

$$
\begin{aligned}
& \text { Upper Layer } \\
& \dot{V}_{U, i j}=G_{C a} C a_{\infty}\left(E_{C a}-V_{U, i j}-V_{e}\right)+G_{K} \theta_{U, i j}\left(E_{K}-V_{U, i j}-V_{e}\right) \\
& +G_{L}\left(E_{L}-V_{U, i j}-V_{e}\right)-S_{U, i j}-k_{0} W\left(\phi_{U, i j}\right) V_{U, i j}+C\left(V_{U, i j}\right) \\
& +D_{L U}\left(V_{L, i j}-V_{U, i j}\right)+F(t) \beta_{i \theta_{1}} \beta_{j \theta_{2}} \\
& \dot{\theta}_{U, i j}=\tau_{\theta}\left(K_{\infty}-\theta_{U, i j}\right) \\
& \dot{S}_{U, i j}=\mu\left(V_{0}+V_{U, i j}\right) \\
& \dot{\phi}_{U, i j}=k_{1} V_{U, i j}-k_{2} \phi_{U, i j} \\
& \dot{V}_{L, i j}=G_{C a} C a_{\infty}\left(E_{C a}-V_{L, i j}-V_{e}\right)+G_{K} \theta_{L, i j}\left(E_{K}-V_{L, i j}-V_{e}\right)+G_{L}\left(E_{L}-V_{L, i j}-V_{e}\right) \\
& -S_{L, i j}-k_{0} W\left(\phi_{L, i j}\right) V_{L, i j}+C\left(V_{L, i j}\right)+D_{U L}\left(V_{U, i j}-V_{L, i j}\right) \\
& +F(t) \beta_{i \theta_{1}} \beta_{j \theta_{2}} \\
& \dot{\theta}_{L, i j}=\tau_{\theta}\left(K_{\infty}-\theta_{L, i j}\right) \\
& \dot{S}_{L, i j}=\mu\left(V_{0}+V_{L, i j}\right) \\
& \dot{\phi}_{L, i j}=k_{1} V_{L, i j}-k_{2} \phi_{L, i j}
\end{aligned}
$$


where the intra layer coupling is defined by the function $C\left(V_{X, i j}\right)=V_{X, i+1 j}+V_{X, i-1 j}+V_{X, i j+1}+$ $V_{X, i j-1}-4 V_{X, i j}, X \in(U, L)$. The inter later coupling strengths are defined by $D_{U L}=D_{L U}=0.05$ with the stimuli forced $F(t)$ applied to the left boundary of the layers. The other parameters are defined as in [20] with only the frequency of the applied electric field considered as the control parameter. Depending on the nodes which are exposed to electric field we can subdivide the discussion in to three categories as

Category-A: Neurons in the upper layer are exposed to electric field.

Category-B: Neurons in the lower layer are exposed to electric field.

Category-C: Neurons in the both the layers are exposed to electric field.

In each category we investigate two cases wherein the first case deals with the study of wave propagation in the network with only electric field while the second case we consider that the neurons are exposed to both electric and magnetic fields.

3.2.1 Neurons in the upper layer are exposed to electric field: In this subsection we considered that the neurons in the upper layer are exposed to an electric field while the lower layer nodes are considered without electric field effects. Under these assumptions we have two sub cases of discussion depending on the magnetic field effects. Fig.13 shows the snapshots of the network (5) considering that the first layer nodes are exposed to electric field effects while the second layer is considered to have no field effects. Clearly the multilayer network shows signs of spiral waves for very less frequency of electric field as shown in Fig.13. A travelling spiral wave is seen for $\omega=$ 0.5 in the right boundary of both the layers while the one in upper layer is not strong and shows signs of low frequency spiking, the one in lower layer is strong and induces similar spiral wave in the left boundary. This spiral wave expands when the frequency is increased to $\omega=0.8$ and when we tried to increase the simulation time to 7000s the spiral waves expand in radius and go outside the network. By making the frequency $\omega=1$, the spiral waves are shown in pars from the left boundary origin while the right top boundary shows travelling wave like behaviour. This is because the nodes in the network under go in chaotic bursting
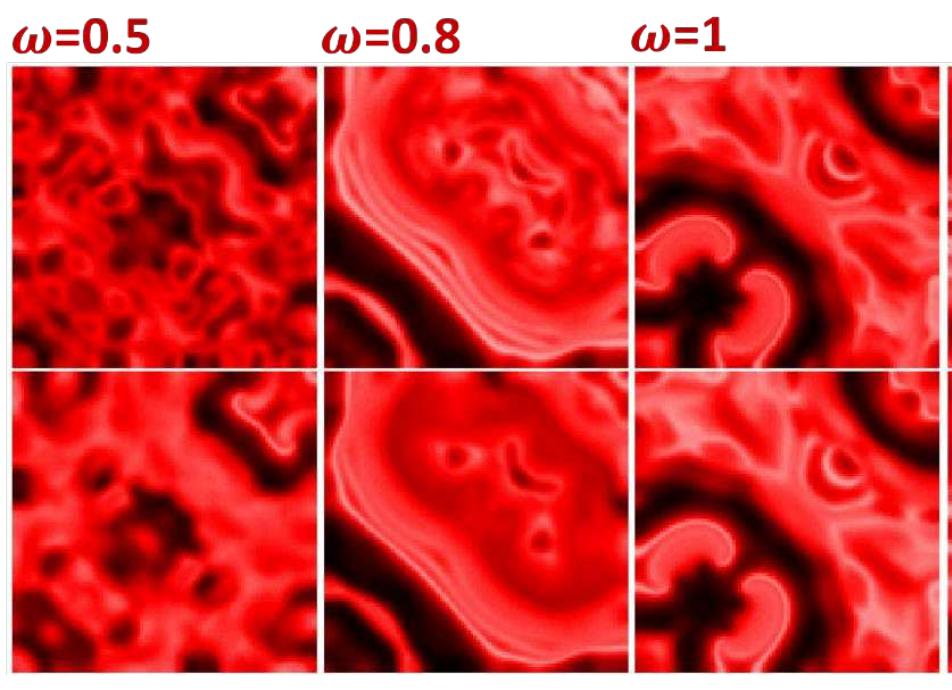

$\omega=1.2$

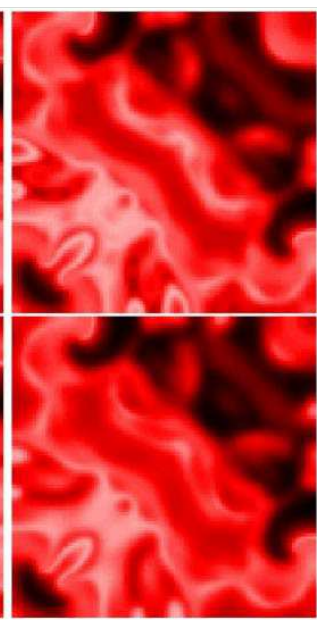

$\omega=1.3$

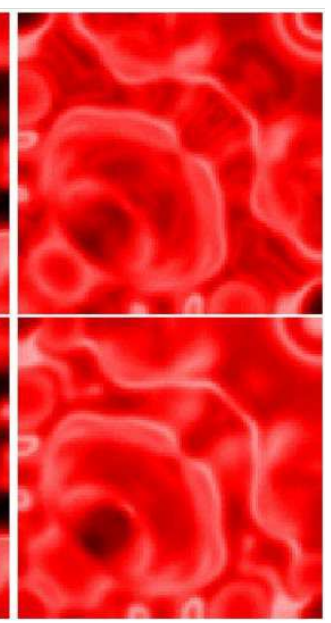


Fig.13: Wave propagation in the two-layer network (5) considering the ML-EM neurons in the upper layer are exposed to electric field while the lower layer is considered to have no field effects. The snapshots are captured for 5000s for different values of $\omega$.

In Fig.13, the nodes in both the layers are not exposed to magnetic field while in Fig. 14 we have considered that the nodes in both the layers are with magnetic flux coupling effect and only the nodes of the first layer is exposed to electric field. It is clear from Fig.14 that the magnetic field coupling induces spiral waves in the network for much lower frequencies of the applied electric field. The spiral waves are seen in both the layers for $0.1 \leq \omega \leq 0.5$ and the network shows local pools of neurons with positive spiking which are soon dissipated when the frequency if increased to $\omega=0.8$. Hence, we could conclude that the spiral waves are exhibited for much lower frequency regions when exposed to the external magnetic flux because of the nodes exhibiting chaotic bursting.
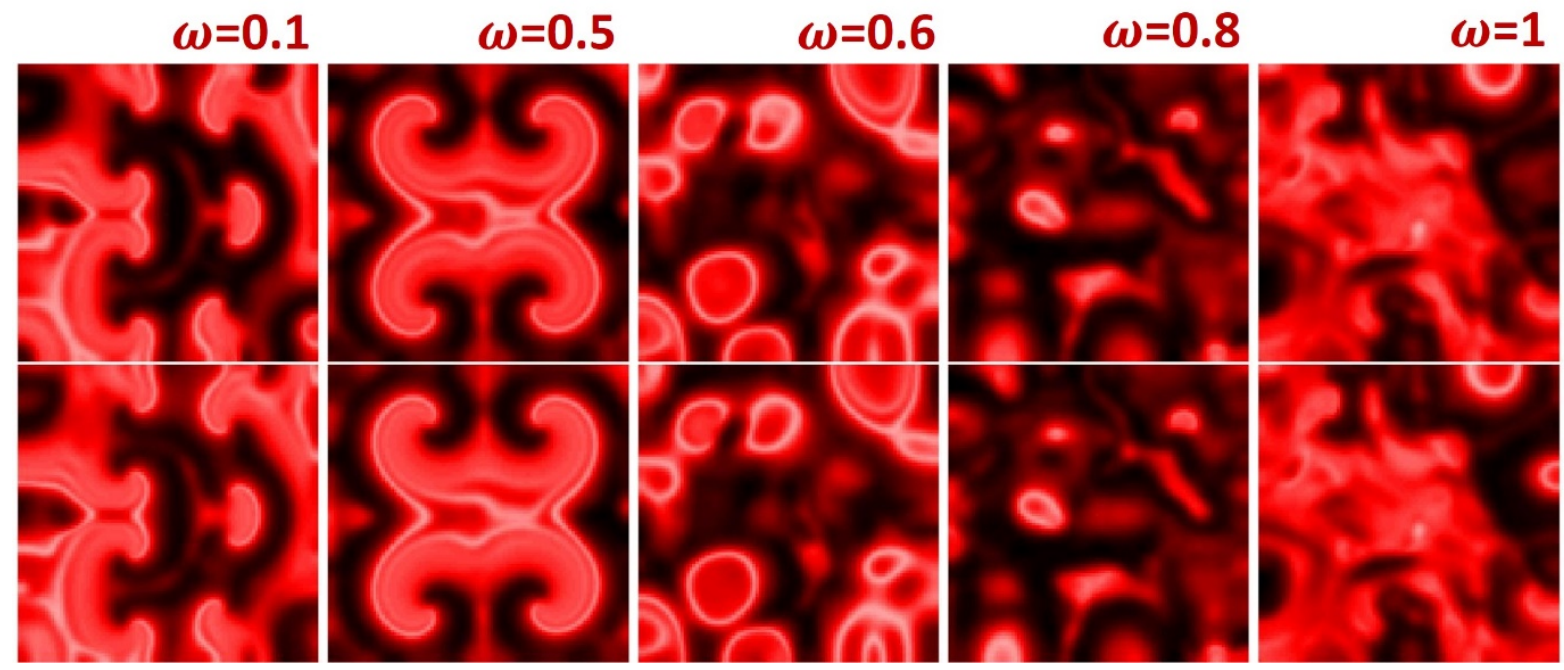

Fig.14: Wave propagation in the two-layer network (5) considering the ML-EM neurons in the upper layer are exposed to electric and magnetic fields while the lower layer is considered to have magnetic field effects. The snapshots are captured for 5000s for different values of $\omega$.

3.2.2 Neurons in the lower layer are exposed to electric field: In the previous subsection we considered that the nodes in the upper layer are exposed to both electric and magnetic field effects. In this subsection we considered that the nodes in the lower layer are exposed to both the fields and under this case, we have two scenarios to investigate. In the first scenario we consider that the nodes in upper layer are not exposed to magnetic coupling while in the second scenario we consider that the nodes are exposed to magnetic field coupling. Fig. 15 shows the snapshots of the network considering no magnetic field coupling. The network shows spiral waves for two sets of electric field frequencies. In the first set we consider low frequency electric field $(\omega<0.5)$ and the spiral waves are seen with asymmetric pairs of small armed spirals originating in all four corners of the first layer while the same is seen only near the origin in the second layer. Thus, we could say that the electric field largely affects the formation of spiral waves as the lower layer which is exposed to electric field has spiral waves only near the origin. The same can be verified for $\omega=0.5$, where the first layer has smaller spiral seeds near the origin while the second layer is totally turbulent 
with no wave propagation. The first layer loses the spiral waves due to the local coupling between the layers. When the frequency is increased to $\omega=1$, the intra layer coupling induces spiral waves in the lower layer supressing the effects of the electric field. Further increasing the frequency to $\omega=1.5$, the spiral waves are dissipated in both the layers which are due to the nodes existing area of chaotic bursting. For $\omega=1.8$ we have a brief period of spiral seeds formed in the three corners of the network which are identical in both the layers. Further increasing the frequency of the electric field, the network goes in to idle where the nodes are now locked to either a positive of a negative amplitude.
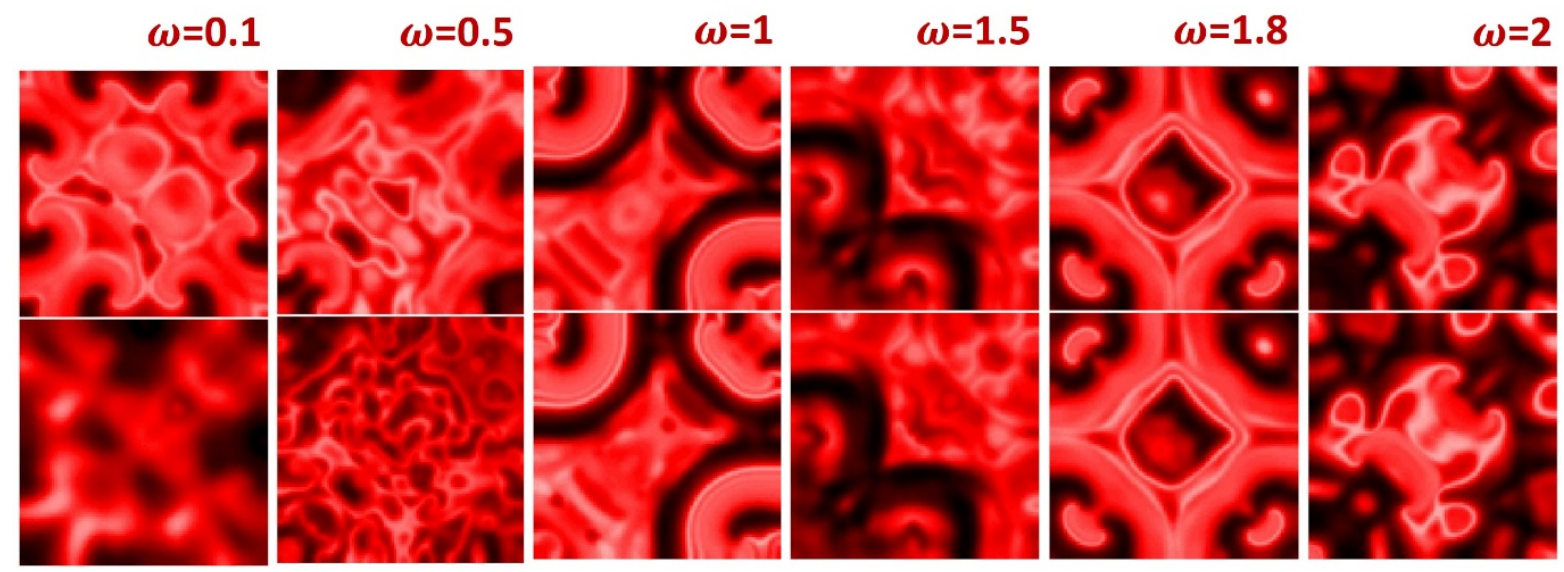

Fig.15: Wave propagation in the two-layer network (5) considering the ML-EM neurons in the lower layer are exposed to electric field while the upper layer is considered to have no field effects. The snapshots are captured for 5000s for different values of $\omega$.

Having discussed about the spiral waves formed in a network where the nodes in the upper layer no field effects, we are now considering a magnetic field coupling in the nodes in both the layers. The snapshots for the network are shown in Fig.16 and we could see that the spiral waves are formed at the center of the network for $\omega=0.1$. These spiral waves are seen in both the layers which are identical as against only in the first layer in a no-field effect upper layer network shown in Fig.15. This is because of the magnetic field coupling overruling the electric field. But increasing the frequency to $\omega=1$ these spiral seeds are dissipated. The re-emergence of spiral waves are not seen in the range $0.5 \leq \omega \leq 2.7$ but increasing the electric field frequency to $\omega=$ 3 , the spiral waves are formed in both the layers but these spiral waves divided in to symmetric pairs forming their local pools of excitation because of the increase in frequency of electric field to $\omega=5$. Increasing the frequency to $\omega=7$ the divided spiral waves are further pushed towards the left and right boundaries with their orientation affected by the applied stimuli. These spiral waves originating from the center of the network are now consolidated to form a single large spiral seed which soon disappears, and the nodes go unstable when the frequency increased from 12 to 12.5 . 

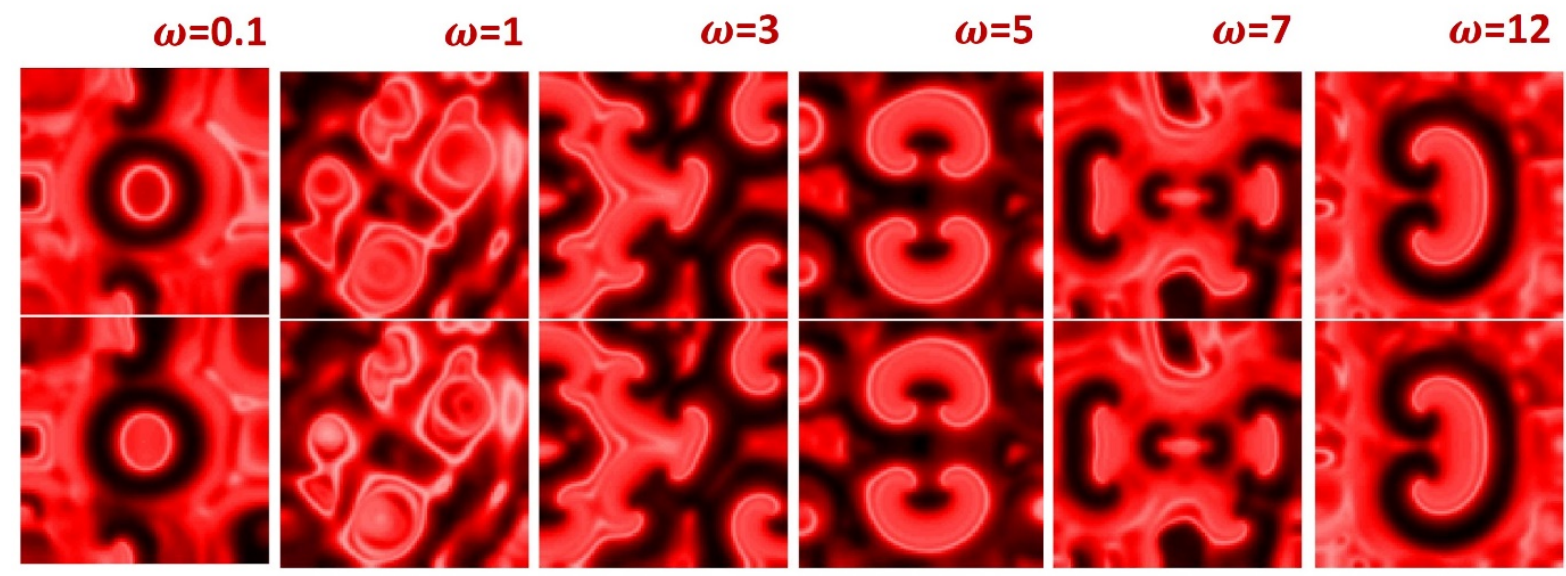

Fig.16: Wave propagation in the two-layer network (5) considering the ML-EM neurons in the lower layer are exposed to electric and magnetic fields while the upper layer is considered to have magnetic field effects. The snapshots are captured for 5000s for different values of $\omega$.

3.2.3 Neurons in both layers are exposed to the electric field: In this section we will investigate the wave propagation when both the layers are exposed to electric fields. Two sub cases are considered where we consider the layers to have only electric field effects or the layers will have both electric and magnetic field effects. In Fig. 17 we considered that the nodes in the layers are exposed to only electric field effects. The nodes show random behaviour and the applied stimuli is dissipated close to the region where it is applied from for electric field frequency $\omega \leq 0.5$. When the frequency of the applied field is increased, the network shows signs of wave propagation and for $\omega \leq 0.8$ the network exhibits traveling wave like propagation. For $\omega=1$, the network shows spiral waves with rotating spiral waves seen near the origin of the network and this wave travels towards the right boundary splitting in to spiral waves in opposite directions. Both the layers show similar behaviour confirming that the coupling between them is intensified due to the synchrony in applied electric field. Further increase in the frequency of excitation results in the network dissipating the waves and resulting in larger pools of nodes locked to their excited voltages.
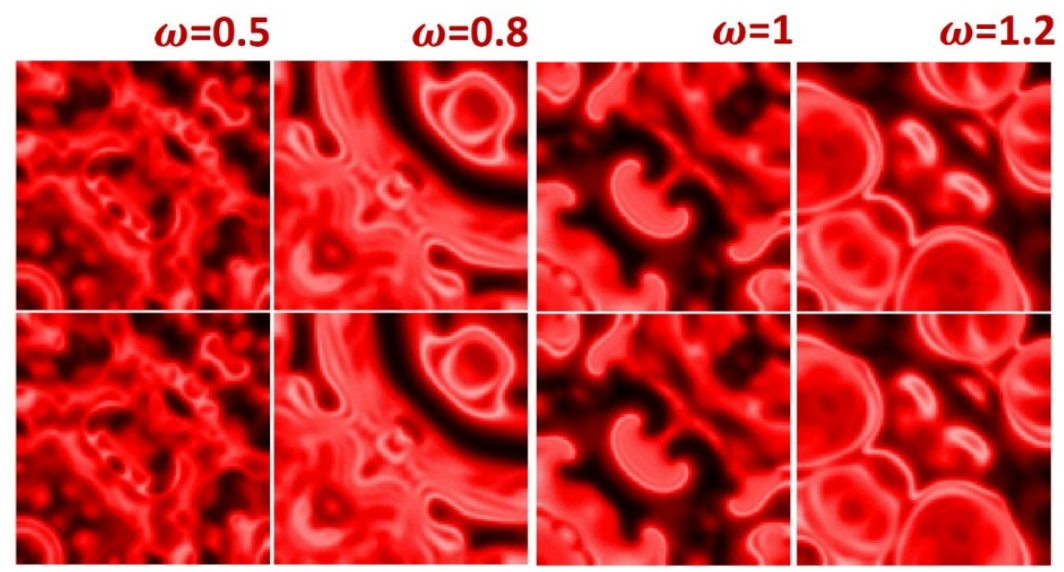
Fig.17: Wave propagation in the two-layer network (5) considering the ML-EM neurons in both the layers are exposed to electric fields. The snapshots are captured for 5000s for different values of $\omega$.

In the second case of this subsection, we consider both the layers to have electric and magnetic field effects. Fig.18 shows the snapshots of the network for various values of electric field frequency. Even for a very low frequency of $\omega=0.001$, the network shows rotating spiral waves in the center of both the layers and a terminal spiral wave near the right boundary. These spiral wave structures are seen up to a frequency of $\omega=1$. When comparing Fig.18 with Fig.17 we could confirm that the magnetic field coupling plays a significant role in the spiral wave existence and has more effect on the network compared to the electric field intensity. When the frequency is increased further the spiral waves are pushed to the corner of the network until $\omega=5$ but again re-emerges from the left boundary while the spiral waves separates to two different spiral pools in the right boundary. Further increase in the frequency drives the network to instability. Thus the range of electric field frequency supporting spiral wave propagation is larger when nodes are exposed to magnetic flux coupling.

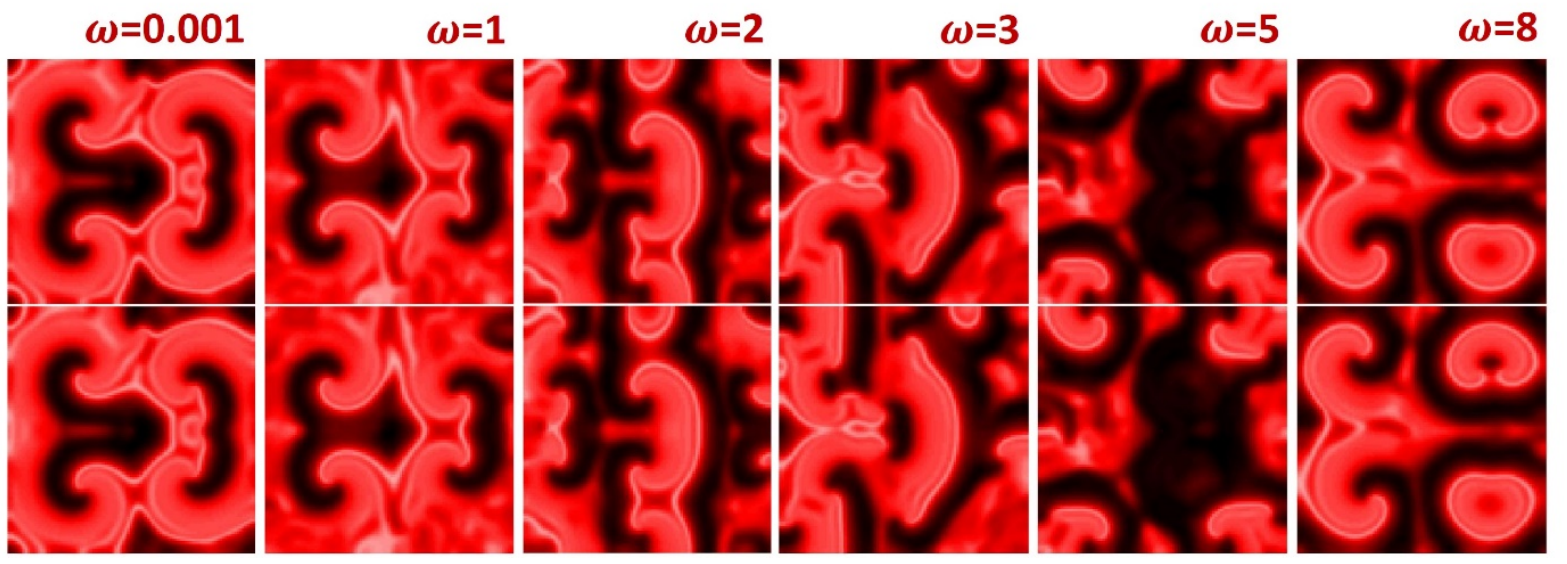

Fig.18: Wave propagation in the two-layer network (5) considering the ML-EM neurons in both the layers are exposed to electric and magnetic fields. The snapshots are captured for 5000s for different values of $\omega$.

\subsection{Network performance in a three-layer network:}

In this section we will discuss about the wave propagation in a three-layer network constructed as defined in (6). The stimuli setting are as in (5). while the intra layer coupling is defined as $C\left(V_{p, i j}\right)=D_{q}\left(V_{p, i+1 j}+V_{p, i-1 j}+V_{p, i j+1}+V_{p, i j-1}-4 V_{p, i j}\right) \quad$ where $p \in(U, M, L)$ and $q \in$ $(1,2,3)$. It is assumed that all the three layers are exposed to electric field while the nodes are not exposed to magnetic field in case-a and are exposed to magnetic field in case-b. For case-a, we consider that $k_{0}=0$ and the entire model in (6) changes to a third order system as the fourth state is not considered due to the flux effects neglected. Fig.18 shows the snapshots of the three-layer network (6) captured for 1000 s considering only electric field effects in all the three layers. For very low frequencies of electric field $(\omega<0.5)$, the stimuli applied to the left boundary of the network do not have much impact on the layers and hence there are no spiral seeds seen. This is 
because the nodes are in chaotic bursting and hence oscillate randomly. When we increased the frequency to $\omega=1$, the nodes now are in periodic spiking resulting in spiral waves in all the three layers. Further increasing $\omega=1.2$, some nodes in the right boundary enter a quasi-periodic state due to the applied excitation resulting in the spiral waves being pushed towards the periodic nodes in the left boundary. The nodes are periodic in the left boundary because of the applied stimuli. Slowly this scenario changes and the nodes in the left boundary go in a quasi-idle state while the nodes in the right boundary enter periodic state and hence showing small spiral seeds for $\omega=1.5$. For $\omega=1.8$, two small spiral pools in opposite directions are seen in the network which are pushed towards the boundary as we increase the frequency to $\omega=2$ after which further increase in the frequency drives the network to instability. Thus, by comparing Fig.17 and Fig.19, we could show that as the number of layers increase, the electric field frequency range affecting the wave propagation increases and thus resulting in hazardous spiral waves in the network.

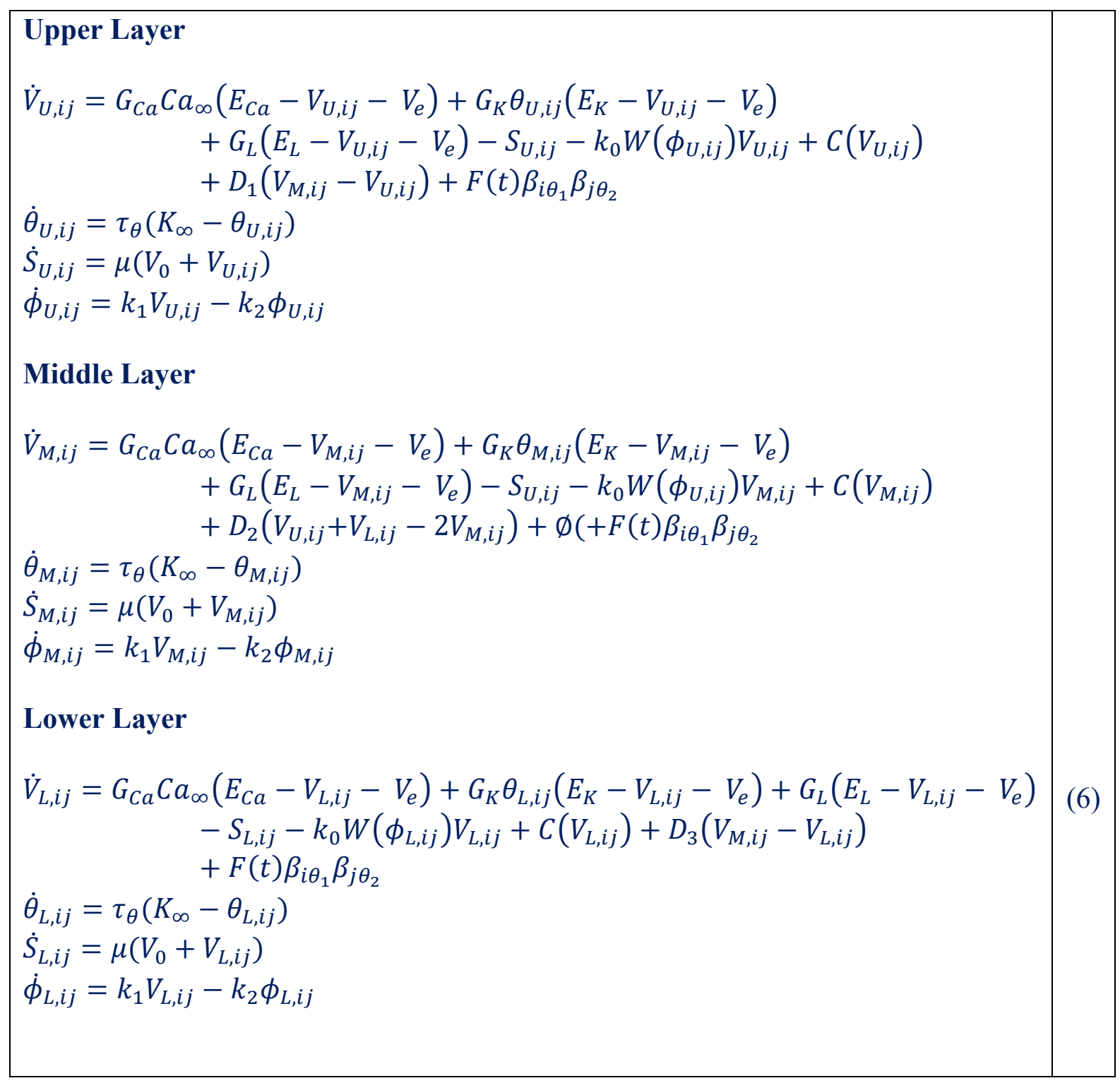




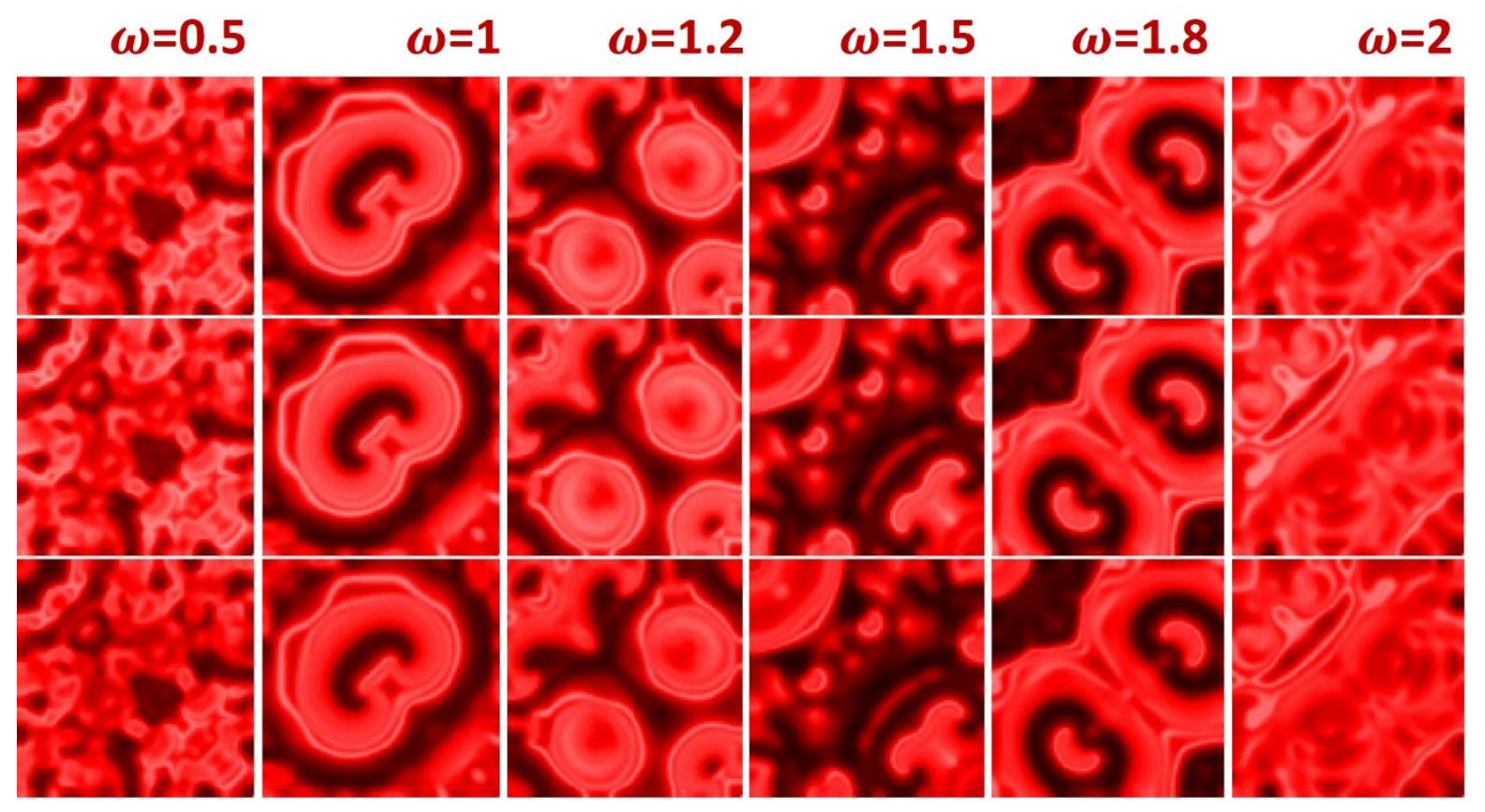

Fig.19: Wave propagation in the three-layer network (6) considering the ML-EM neurons in all the layers are exposed to electric fields. The snapshots are captured for 1000s for different values of $\omega$.

Now consider that the neurons in all the three layers are exposed to both electric and magnetic fields, we investigate the wave propagation phenomenon by applying a periodic stimulus to the left boundary of the network as shown in Fig.20. Interestingly, when the electric field excitation frequency $\omega<0.8$, the network doesn't show spiral wave seeds as like Fig.8. When the frequency is increased to $\omega=1$, spiral waves are seen generated from the right boundary and slowly consolidated in the center of the layers for $\omega=2$. This phenomenon of spiral wave generation and consolidation in the center as asymmetric pairs are seen up to $\omega=3$. This process reverses to left boundary when we increase the frequency to $\omega=4$ and this is because of the cycles of periodic stimuli and electric field phase changes. For $\omega>5$ the spiral waves are seen from the left boundary and move in the direction of the stimuli. Spiral wave pairs are seen in the center of the network when the electric field frequency $\omega>7$. Such spiral waves are not supressed even when we tried changing the value of the flux coupling constant $\left(k_{0}\right)$ confirming that the nodes are in some phase lock mode with the applied stimuli. This lock is broken when we change the stimuli frequency by a slight value to 0.02. Interestingly the spiral waves are seen in the network for electric field frequency $\omega=30$ after which the network goes unstable. Hence, we could again show that irrespective of the number of layers the magnetic field coupling drastically increases the range of electric field frequency supporting spiral waves in the network. 


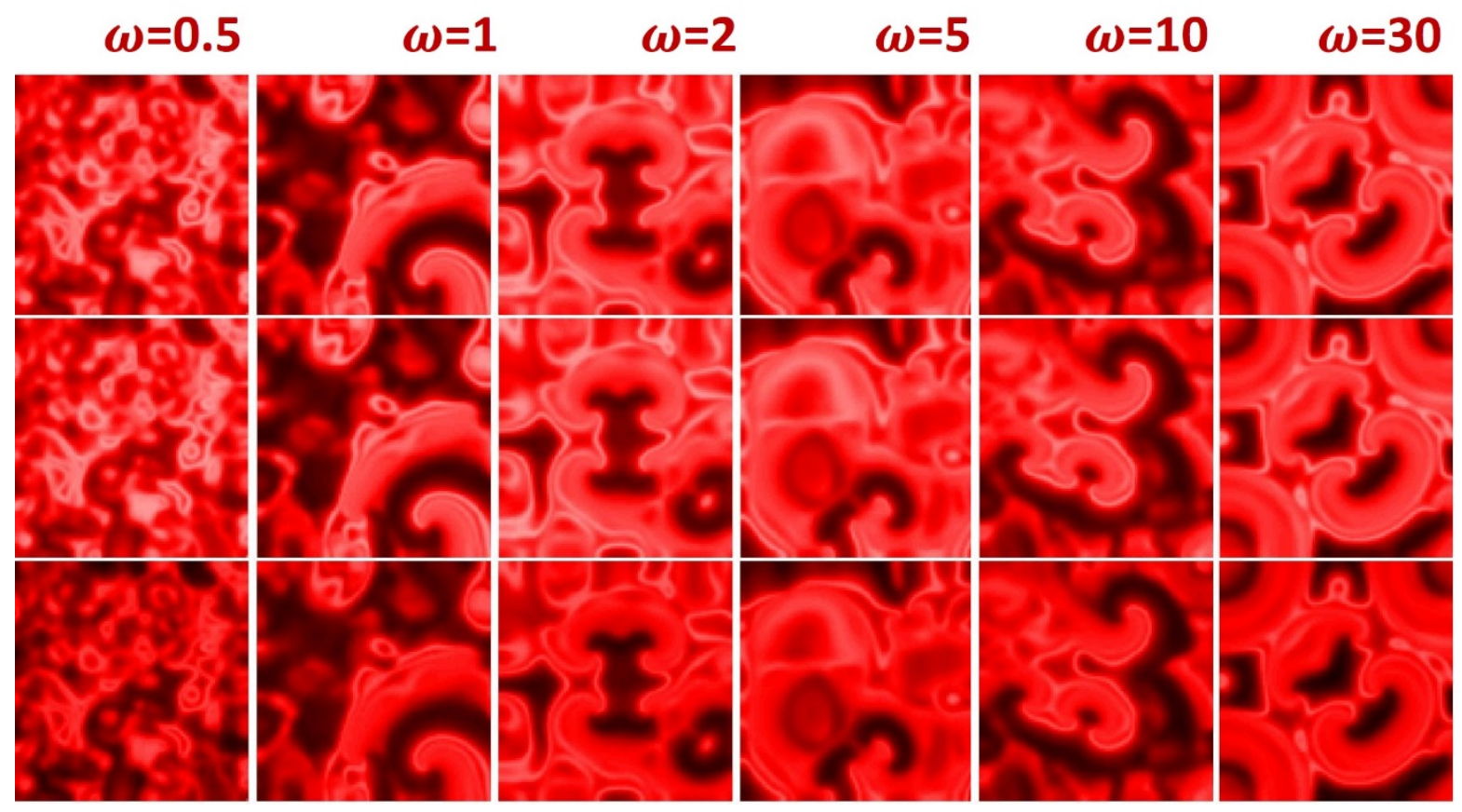

Fig.20: Wave propagation in the three-layer network (6) considering the ML-EM neurons in all the layers are exposed to electric and magnetic fields. The snapshots are captured for 1000 s for different values of $\omega$.

\section{Supressing spiral waves using gaussian noise:}

There have been many studies discussing noisy neuronal networks and their benefits in information transfer $[25,26]$. It is considered nontrivial to use white noise as an additional current term because the noisy activation kinetics can lead to different qualitative behaviours of a neuron [27]. Hence, as discussed in [28,29], we use a Box-Mueller type white noise as an additional current term and see the effect in suppressing spiral waves in different types of network. Generally, a Box-Mueller type white noise is defined by a mathematical form

$$
\zeta(t)=(-(4 \delta / h) \ln (a))^{0.5} \cos (2 \pi b)
$$

where $[a . b]$ are random numbers between [0.1], $\delta$ is the variance of the noise and $h$ is the step size of the solver. Now our aim is to include this noise as a current term in the neuron ML-EF neuron model and study the network behaviour in single and multiplex networks. For the discussion we have considered that the nodes are exposed to both electric and magnetic field effects.

\subsection{Effect of noise in a single layer network:}

In the first scenario for discussion, we consider the Box-Mueller type noise as a current term in a network of ML-EF nodes defined in (8). We have considered that the stimuli $(F(t)=A \sin (\Omega t))$ is applied from the left boundary with amplitude $A=0.1$ and frequency $\Omega=0.01$. The system parameters are considered as in [20] and the nodes are assumed to be exposed to both electric and magnetic fields. The frequency of the electric field excitation is fixed as $\omega=1.2$ for which the single layer network shows spiral waves as in Fig.12. Our aim is to investigate the effect of the 
noise on the wave propagation in the network and hence we considered the variance $\delta$ as the control parameter. The snapshots are captured for 5000s and the numerical simulation was done using RK4 considering step size as 0.05 as in Fig.21.

$$
\begin{aligned}
& C \dot{V}_{i j}=G_{C a} C a_{\infty}\left(E_{C a}-V_{i j}-V_{e}\right)+G_{K} \theta_{i j}\left(E_{K}-V_{i j}-V_{e}\right) \\
& +G_{L}\left(E_{L}-V_{i j}-V_{e}\right)-S_{i j}-k_{0} W\left(\phi_{i j}\right) V_{i j}+C\left(V_{i j}\right) \\
& +F(t) \beta_{i \theta_{1}} \beta_{j \theta_{2}}+\zeta(t) \\
& \dot{\theta}_{i j}=\tau_{\theta}\left(K_{\infty}-\theta_{i j}\right) \\
& \dot{S}_{i j}=\mu\left(V_{0}+V_{i j}\right) \\
& \dot{\phi}_{i j}=k_{1} V_{i j}-k_{2} \phi_{i j}
\end{aligned}
$$

We started the investigation by consider the noise variance as $\delta=0.0001$ and the network shows multiple spiral waves in the entire layer and the same is the case when we increased the noise variance to $\delta=0.001$. But when the variance is increased to $\delta=0.01$, the spiral waves are seen dissipated and we could see only few spiral seeds in the network which are dominated by the dissipated waves which are because of the nodes showing chaotic behaviour. By further increasing $\delta=0.03$, the spiral waves are eliminated from the network and even increasing the frequency of electric field excitation doesn't have any impact on the nodes. Thus, by exposing the nodes to noise, the spiral waves are supressed in a single layer network.
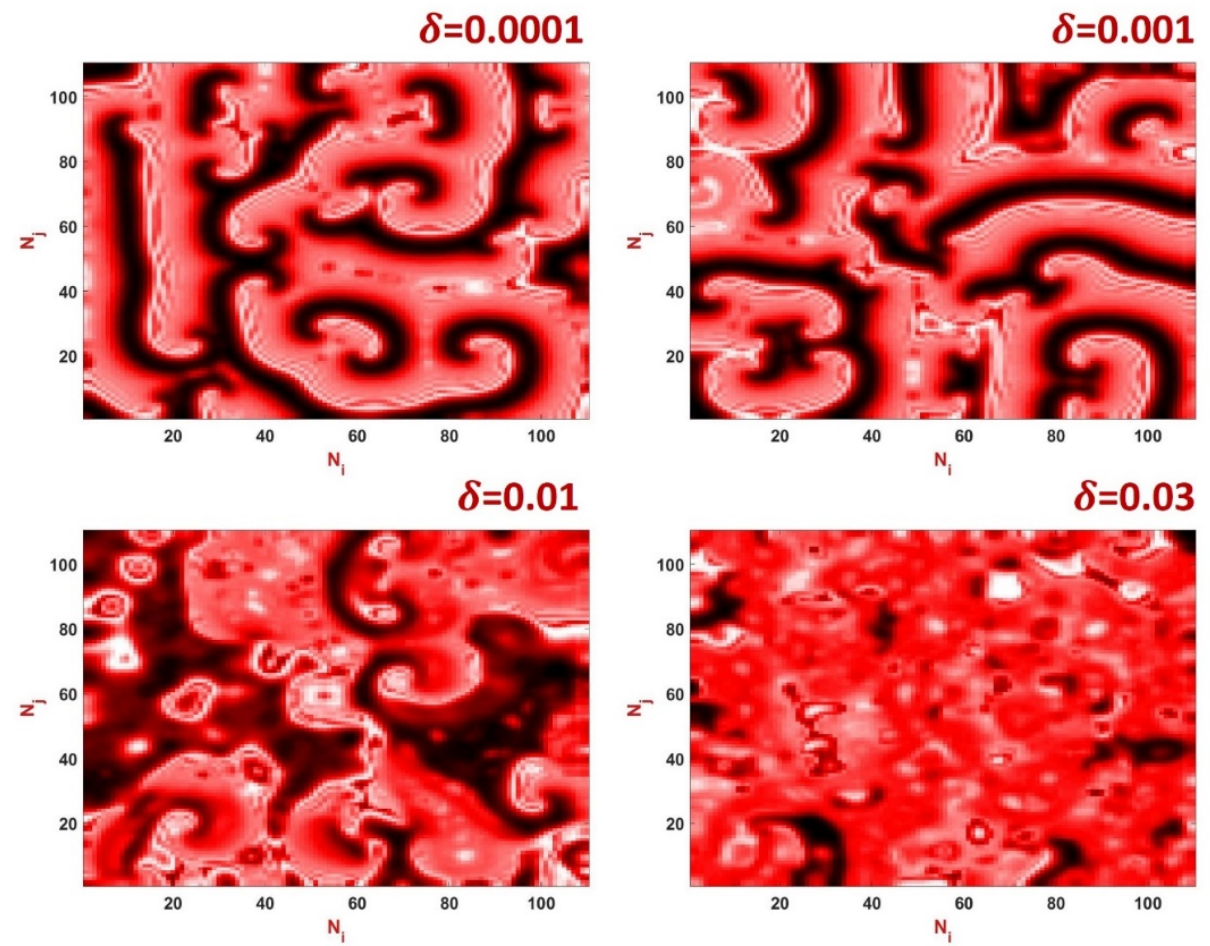

Fig.21: Effect of noise on the wave propagation in a single layer network. The noise variance $\delta$ is considered as the control parameter.

4.2 Effect of noise in a two-layer network: 
By showing that inclusion of white noise in the local kinetics of the nodes in a single layer network, we could supress spiral waves, our interest is now to study the noise effects on multi-layer network. To start with, we have considered the two-layer network (11) with the Box-Mueller type white noise and the mathematical model is defined as,

\begin{tabular}{|c|c|}
\hline 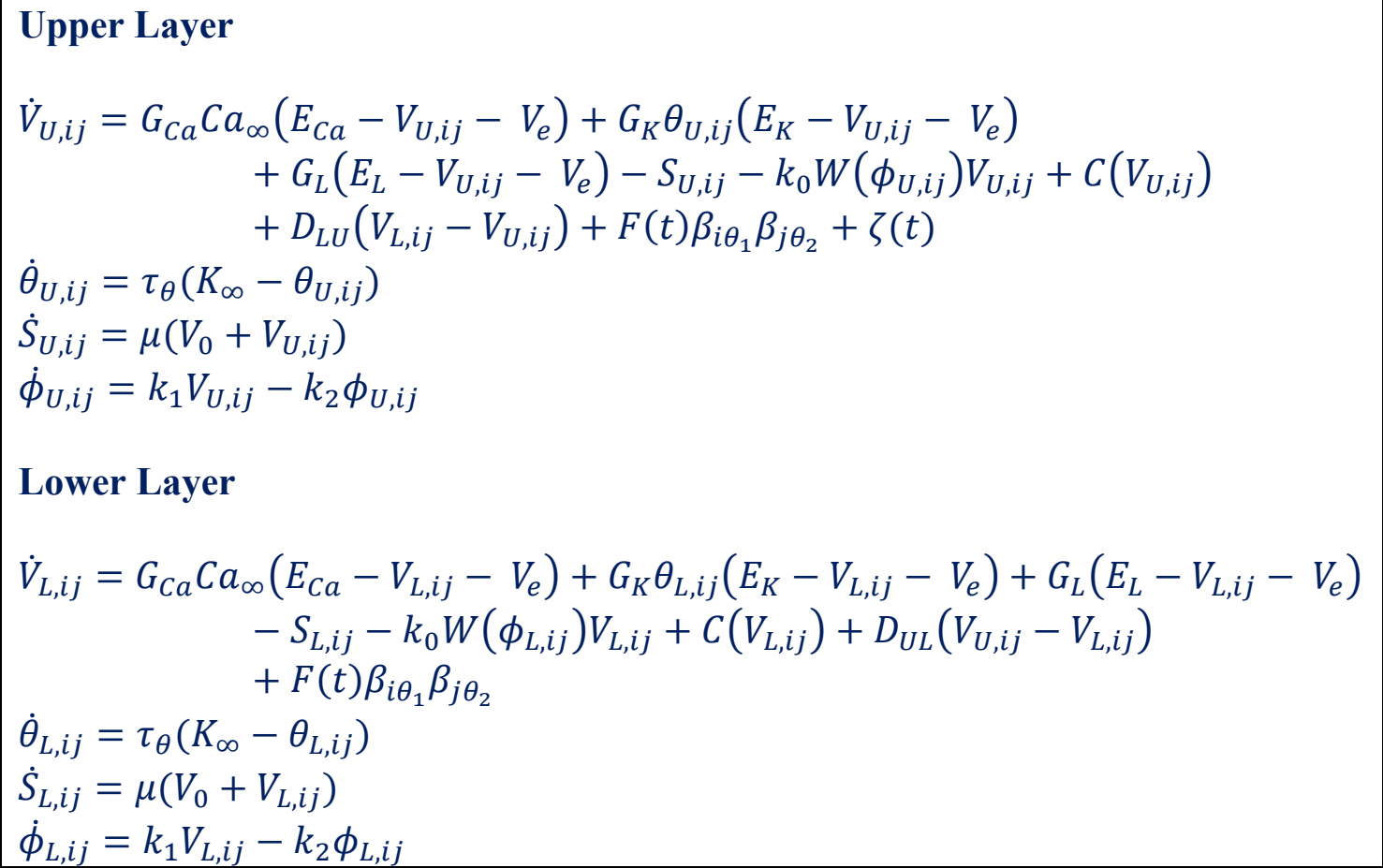 & (9) \\
\hline
\end{tabular}

For this investigation we have assumed that the nodes in both the layers are exposed to both electric and magnetic fields and the stimuli settings are as used in Fig.14. We could see that the spiral waves seen in the network without noise (Fig.18) are now pushed towards the right boundary for a small noise variance of $\delta=0.0001$. Further increasing the noise variance, the spiral waves are dissipated except for small rotating spiral seed near the origin. Spiral waves are eliminated when the noise variance is increased to $\delta=0.0003$. Hence, we could conclude that the effect of noise on two-layer network is more than that of single layer network. This is because that the noise current added to nodes in both the layers drives each other due to the inter layer coupling. 


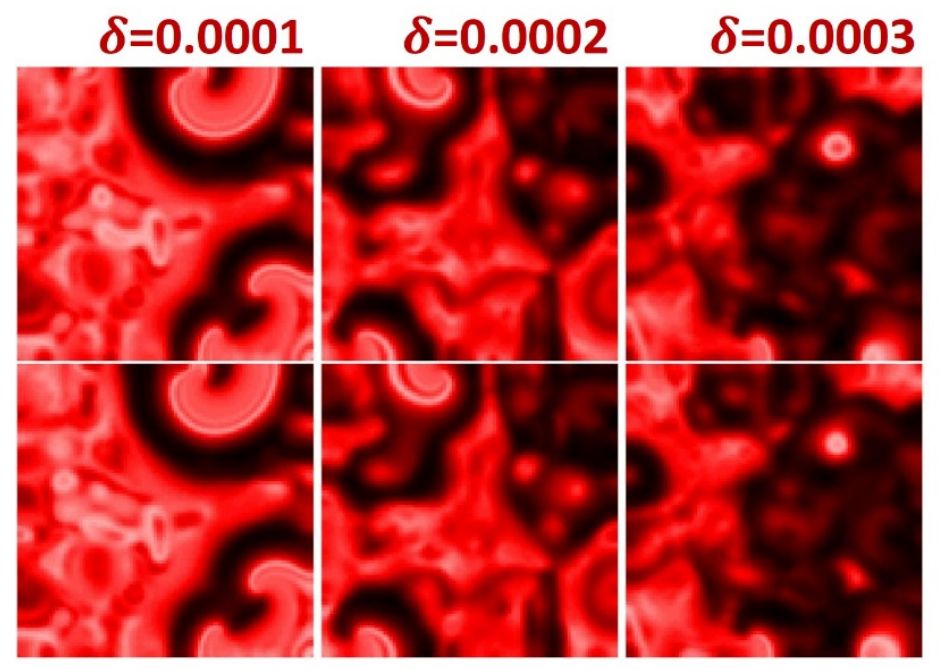

Fig.22: Effect of noise on the wave propagation in a two-layer network. The noise variance $\delta$ is considered as the control parameter. The electric field intensity is fixed as $\omega=1$.

\subsection{Effect of noise in a three-layer network:}

After investigating the noise effects on the two-layer network and showing that a very less noise variance can supress spiral waves in the network, we wish to confirm it in a three-layer network. Hence, we constructed a three-layer network (6) by including the noise current term in each node. The other parameter and stimuli setting are same as used in (6). We have considered the electric field frequency to be $\omega=1.2$ and varied the noise variance.

\begin{tabular}{|c|c|}
\hline 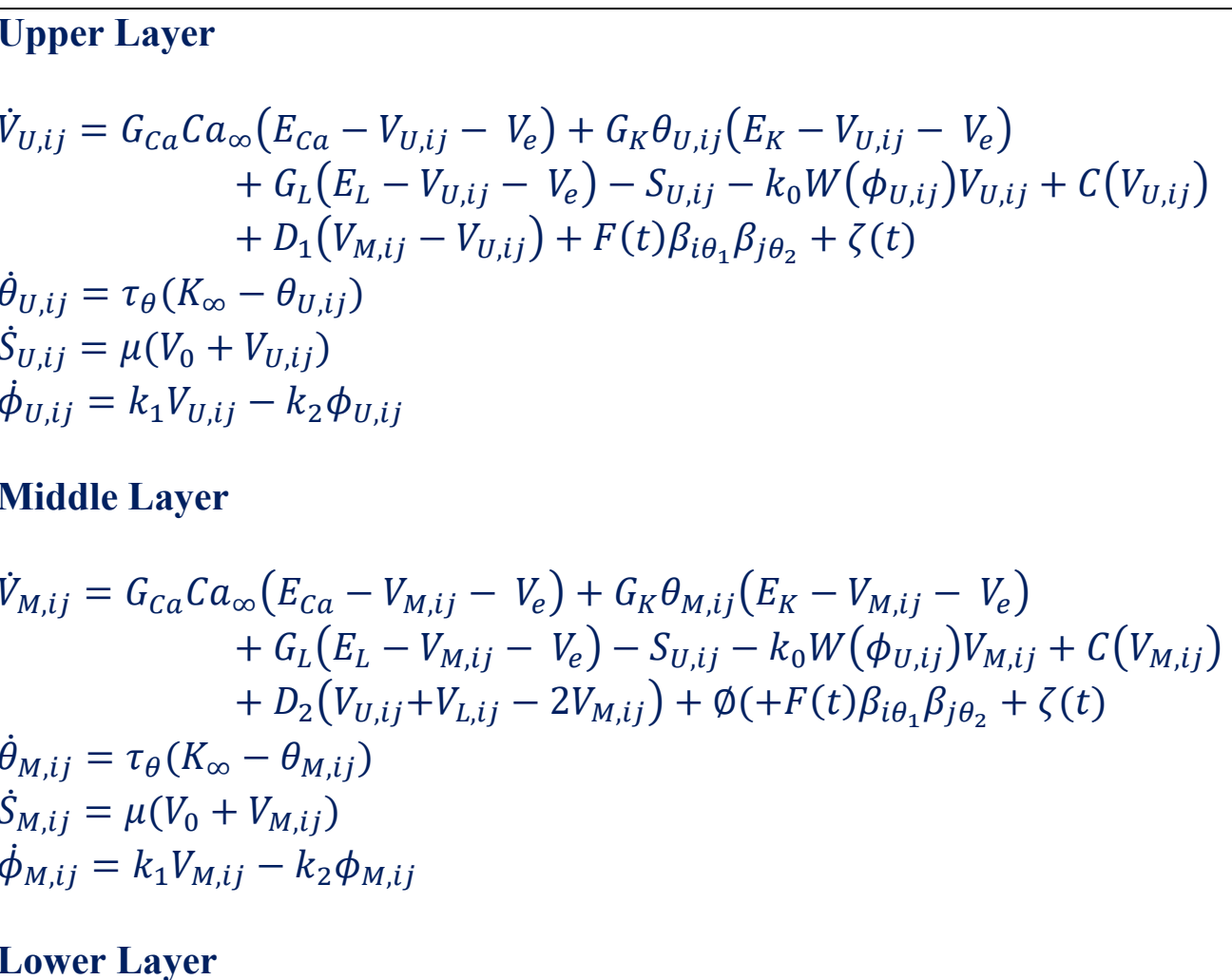 & (10) \\
\hline
\end{tabular}




$$
\begin{aligned}
& \dot{V}_{L, i j}=G_{C a} C a_{\infty}(\left.E_{C a}-V_{L, i j}-V_{e}\right)+G_{K} \theta_{L, i j}\left(E_{K}-V_{L, i j}-V_{e}\right)+G_{L}\left(E_{L}-V_{L, i j}-V_{e}\right) \\
& \quad-S_{L, i j}-k_{0} W\left(\phi_{L, i j}\right) V_{L, i j}+C\left(V_{L, i j}\right)+D_{3}\left(V_{M, i j}-V_{L, i j}\right) \\
& \quad+F(t) \beta_{i \theta_{1}} \beta_{j \theta_{2}}+\zeta(t) \\
& \dot{\theta}_{L, i j}= \tau_{\theta}\left(K_{\infty}-\theta_{L, i j}\right) \\
& \dot{S}_{L, i j}=\mu\left(V_{0}+V_{L, i j}\right) \\
& \dot{\phi}_{L, i j}=k_{1} V_{L, i j}-k_{2} \phi_{L, i j}
\end{aligned}
$$

In Fig.23, we have captured the snapshots of the network for 1000s for various values of the noise variance $\delta$. For easy reference we have also considered the no noise case by keeping $\delta=0$. The spiral waves in the network are affected even for a very small noise variance of $\delta=0.0001$ but are not supressed. Hence, we tried increasing the noise variance to of $\delta=0.0002$ for which the spiral seeds are seen at the top and bottom of each layer in the network. These spiral waves are not affected by the frequency of the electric field till $\omega=10$ after which the network goes unstable. Hence, we increased the noise variance further to $\delta=0.0005$ and now the spiral waves are largely suppressed, and a simple rotating spiral seed is seen near the origin. For $\delta=0.0007$ the spiral waves are completely supressed, and the network is now in complete turbulence. When comparing the two layer and the three-layer network we could see that the noise variance required to supress spiral waves in the three-layer network is higher than that required for suppression in two-layer network.

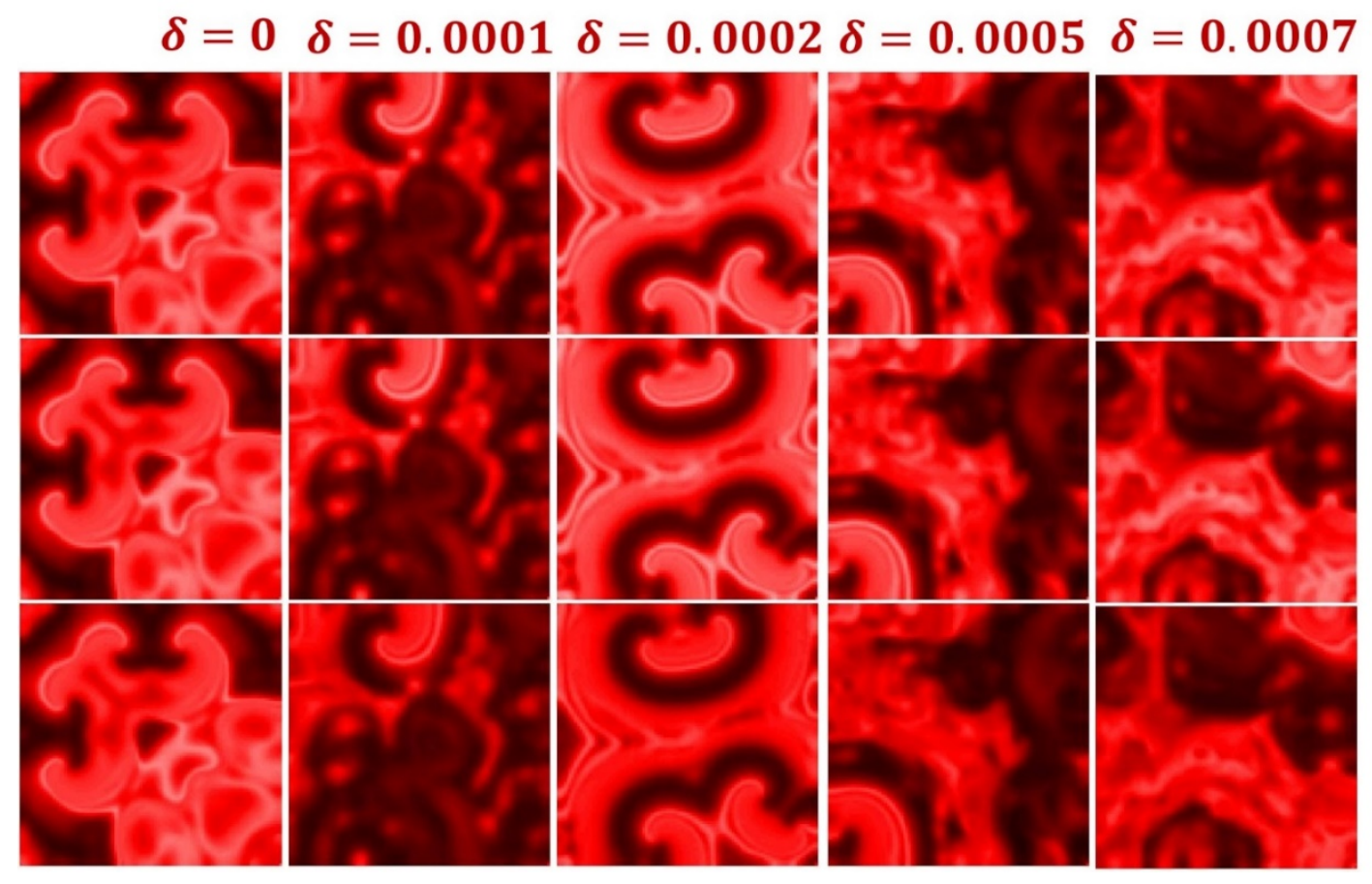

Fig.23: Effect of noise on the wave propagation in a two-layer network. The noise variance $\delta$ is considered as the control parameter. The electric field intensity is fixed as $\omega=1.2$. 


\section{Conclusion}

In our earlier work on Morris - Lecar neuron model, we have considered only magnetic field effects. But the published literature has shown that electric field effects also have significant impact on the dynamics of neurons. Hence, we modified the M-L neuron model to show electric and magnetic field effects. By applying a constant electric field of small amplitude, we could show that the chaotic bursting phenomenon is completely lost. When periodic sinusoidal electric field is applied for very low frequency, the modified M-L model shows cascading bifurcation. We could also show a frequency locked state for lower frequency of electric field. To investigate the Network dynamics of the ML-EM network we constructed single and multiple lattice array and discussed the spatiotemporal behaviour. In a single layer lattice array we discussed two scenario. In the first scenario only, electric field is applied to the network and we could show the existence of spiral wave for frequency between $[0.95,1]$. In the second scenario we have applied both electric and magnetic fields and could prove that a magnetic field does not affect the spiral waves in the network. In the second type of network, a two-layer lattice array of ML-EM neurons are considered Under this we have three different categories of discussion. In category - A we consider that neurons in upper layer all exposed to electric field and magnetic field, while the neurons in lower layer are exposed to only magnetic field effects. By such conditions, the spiral waves are seen in the network. For $0.9 \leq \omega \leq 1.2$. In second category the neuron in lower layer are exposed to electric and magnetic fields, while the upper layers have only magnetic field effects. Now the layer shows spiral wave only for lower frequency value of electric field. The range of frequency exhibit spiral wave in the network is $0.1 \leq \omega \leq 0.5$. Then both the layer is exposed to electric effects, we could see that the spiral wave is seen for two different bands of electric field frequencies $0.1 \leq \omega \leq 0.5$ and $0.9 \leq \omega \leq 1.5$. When the layers are exposed to both the electric and magnetic fields, the electric field frequencies inducing spiral wave ranging from a very low frequency of $\omega=0.001$ to a frequency of $\omega=8$. We could note that the frequency change doesn't much affect the spiral wave formation. To further understand the multi-layer network behaviour, we constructed a three-layer network and in the first case all three layer are exposed only to electric field effects. A narrow range of electric field frequencies $1 \leq \omega \leq 1.8$ shows formation of spiral wave. But when we additionally applied magnetic fields the range of $\omega$ increases to $\omega=30$ and the network does not show much impact of $\omega$ as spiral wave are not dissipated. we could confirm that in multi-layer network electric and magnetic fields combined effect increases the range of ' $\omega$ ' supporting spiral wave formation. To investigate the noise effects, we have considered a Box-Muller type Gaussian noise as an additional current term. In a single layer network, we need a much larger noise variant to dissipate the spiral wave compared to the multi-layer networks. We have assumed that the networks are exposed to both electric and magnetic field effects.

Conflict of Interest: The authors declare that they have no conflict of interest.

Funding: Karthikeyan Rajagopal and Balamurali Ramakrishnan have been partially funded by the Research grant of Center for Nonlinear Systems, Chennai Institute of Technology with reference number CIT/CNS/2020/RP-062. 


\section{References}

[1]. Hodgkin.A.L, "The local electric changes associated with repetitive action in a nonmedullated axon". J Physiol 107:165-181,1948.

[2]. Tsumoto K, Kitajima $\mathrm{H}$ et al, "Bifurcations in Morris-Lecar neuron model" Neurocomputing 69:293-316, 2006.

[3]. Congmin Liu, Xuanliang Liu, Shenquan Liu, "Bifurcation analysis of a Morris-Lecar neuron model", Biol Cybern, 108:75-84, 2014.

[4]. M. Lv and J. Ma, "Multiple modes of electrical activities in a new neuron model under electromagnetic radiation," Neurocomputing, vol. 205, pp. 375-381, 2016.

[5]. M. Lv, C. Wang, G. Ren, J. Ma, and X. Song, "Model of electrical activity in a neuron under magnetic flow effect," Nonlinear Dynamics, vol. 85, no. 3, pp. 1479-1490, 2016.

[6]. F. Wu, C. Wang, Y. Xu, and J. Ma, "Model of electrical activity in cardiac tissue under electromagnetic induction," Scientific Reports, vol. 6, no. 1, 2016.

[7]. J. Ma, F. Wu, and C. Wang, "Synchronization behaviors of coupled neurons under electromagnetic radiation," International Journal of Modern Physics B, vol. 31, no. 2, p. $1650251,2017$.

[8]. Y. Wang, J. Ma, Y. Xu, F. Wu, and P. Zhou, "The electrical activity of neurons subject to electromagnetic induction and gaussian white noise," International Journal of Bifurcation and Chaos, vol. 27, no. 2, p. 1750030, 2017.

[9]. F. Wu, C. Wang, W. Jin, and J. Ma, "Dynamical responses in a new neuron model subjected to electromagnetic induction and phase noise," Physica A: Statistical Mechanics and its Applications, vol. 469, pp. 81-88, 2017.

[10]. J. Wu , Y. Xu , and J. Ma, "Lévy noise improves the electrical activity in a neuron under electromagnetic radiation," PLoS One, vol. 12, no. 3, p. e0174330, 2017.

[11]. G. Ren, Y. Xu, and C. Wang, "Synchronization behavior of coupled neuron circuits composed of memristors," Nonlinear Dynamics, vol. 88, no. 2, pp. 893-901, 2017.

[12]. Huang X Y, Troy W C, Yang Q C, et al. Spiral waves in disinhibited mammalian cortex. J Neurosci, 24(44): 9897-9902, 2004.

[13]. Schiff S J, Huang X Y, Wu J Y. Dynamical evolution of spatiotemporal patterns in mammalian middle cortex. Phys Rev Lett, 98(17): 178102, 2007.

[14]. Sridhar S, Sinha S. Controlling spatiotemporal chaos in excitable media using an array of control points. Europhys Lett, 81(5):50002, 2008.

[15]. Tang G N, Deng M Y, Hu B B, et al. Active and passive control of spiral turbulence in excitable media. Phys Rev E, 77(4): 46217, 2008.

[16]. Zhan M, Kapral R. Destruction of spiral waves in chaotic media. Phys Rev E, 73(2): 026224, 2006. 
[17]. Wu N J, Zhang H, Ying H P, et al. Suppression of Winfree turbulence under weak spatiotemporal perturbation. Phys Rev E, 73(6): 060901(R), 2006.

[18]. Ma J, Ying H P, Li Y L. Suppression of spiral waves using intermittent local electric shock. Chin Phys, 16(4): 955-961, 2007.

[19]. Panfilov A V, M"uller S C, Zykov V S. "Elimination of spiral waves in cardiac tissue by multiple electrical shocks". Phys Rev E, 61(4):4644-4647, 2000.

[20]. Rajagopal, K., Moroz, I., Karthikeyan, A., Duraisamy, P. "Wave propagation in a network of extended Morris-Lecar neurons with electromagnetic induction and its local kinetics", Nonlinear Dynamics, 100 (4), pp. 3625-3644, 2020.

[21]. Hu B, Ma J, Tang J (2013) "Selection of Multiarmed Spiral Waves in a Regular Network of Neurons". PLoS ONE 8(7): e69251.

[22]. Feng, Y., Khalaf, A.J.M., Alsaadi, F.E. et al. "Spiral wave in a two-layer neuronal network". Eur. Phys. J. Spec. Top. 228, 2371-2379 (2019).

[23]. Yong Wu, Bing Wang, Xiaoxiao Zhang and Hao Chen, "Spiral wave of a two-layer coupling neuronal network with multi-area channels", International Journal of Modern Physics BVol. 33, No. 29, 1950354 (2019).

[24]. Zhen Wang, Zahra Rostami, Sajad Jafari, Fawaz E. Alsaadi, Mitja Slavinec, Matjaž Perc, "Suppression of spiral wave turbulence by means of periodic plane waves in two-layer excitable media", Chaos, Solitons \& Fractals, Volume 128, 2019,Pages 229-233.

[25]. J. K. Douglass, L. Wilkens, E. Pantazelou, and F. Moss, "Noise enhancement of information transfer in crayfish mechanoreceptors by stochastic resonance", Nature 365, 337 (1993).

[26]. H. A. Braun, H. Wissing, K. Scha"fer, and M. C. Hirsch, "Oscillation and noise determine signal transduction in shark multimodal sensory cells", Nature 367, 270 (1994).

[27]. Finke, C., Postnova, S., Rosa, E. et al. "Noisy activation kinetics induces bursting in the Huber-Braun neuron model". Eur. Phys. J. Spec. Top. 187, 199-203 (2010). https://doi.org/10.1140/epjst/e2010-01284-1.

[28]. Christian Finke, Jürgen Vollmer, Svetlana Postnova, Hans Albert Braun, "Propagation effects of current and conductance noise in a model neuron with subthreshold oscillations", Mathematical Biosciences, Volume 214, Issues 1-2, 2008, Pages 109-121.

[29]. Christian Finke, Jan A. Freund, Epaminondas Rosa Jr, Paul H. Bryant, Hans A. Braun, and Ulrike Feudel, "Temperature-dependent stochastic dynamics of the Huber-Braun neuron model", Chaos 21, 047510 (2011).

[30]. Yan-Qiu Che, Jiang Wang, Wen-Jie Si, Xiang-Yang Fei, "Phase-locking and chaos in a silent Hodgkin-Huxley neuron exposed to sinusoidal electric field", Chaos, Solitons \& Fractals, Volume 39, Issue 1, 2009, Pages 454-462. 
Figures

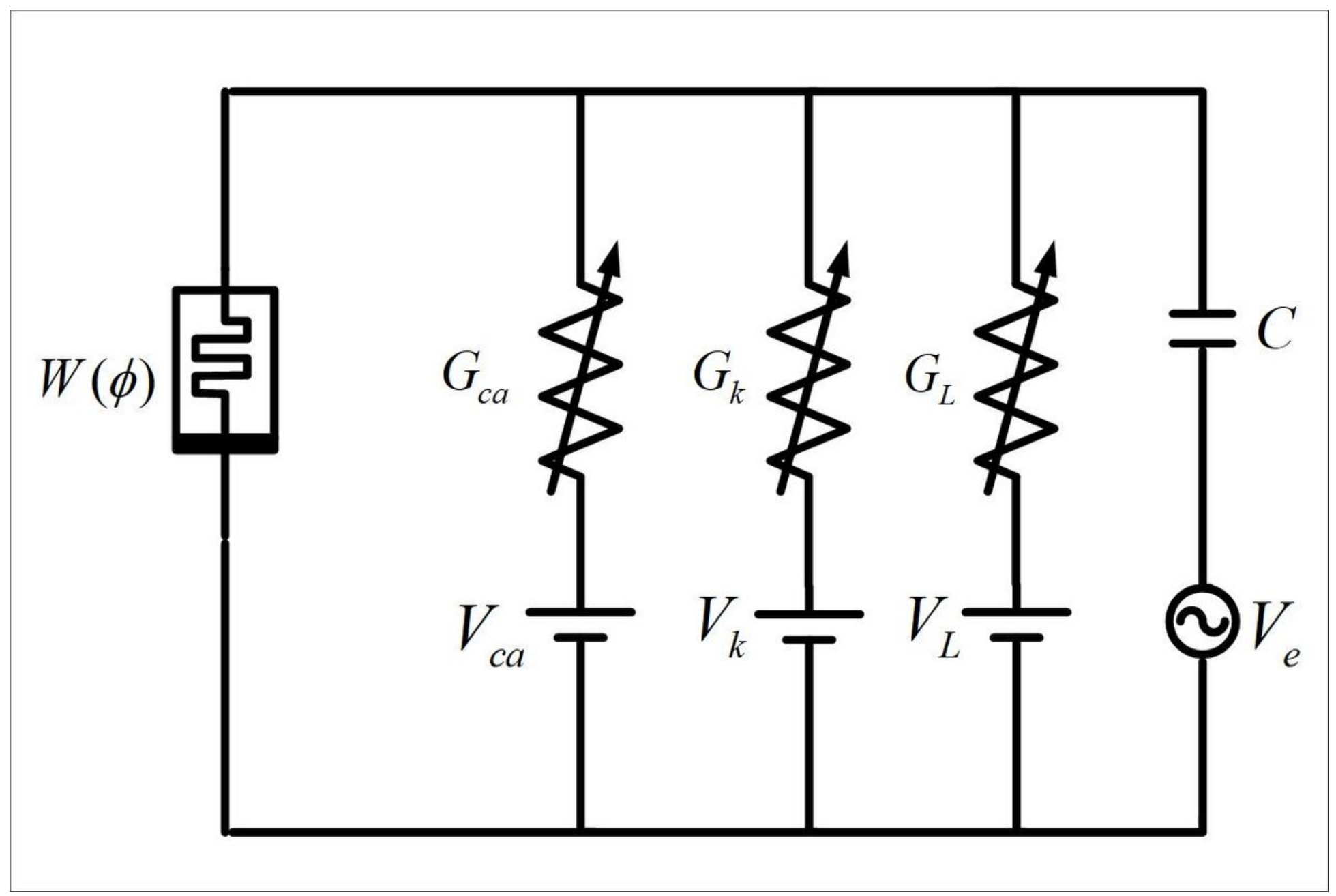

Figure 1

The circuit model of the M-L neuron exposed to electric and magnetic fields 


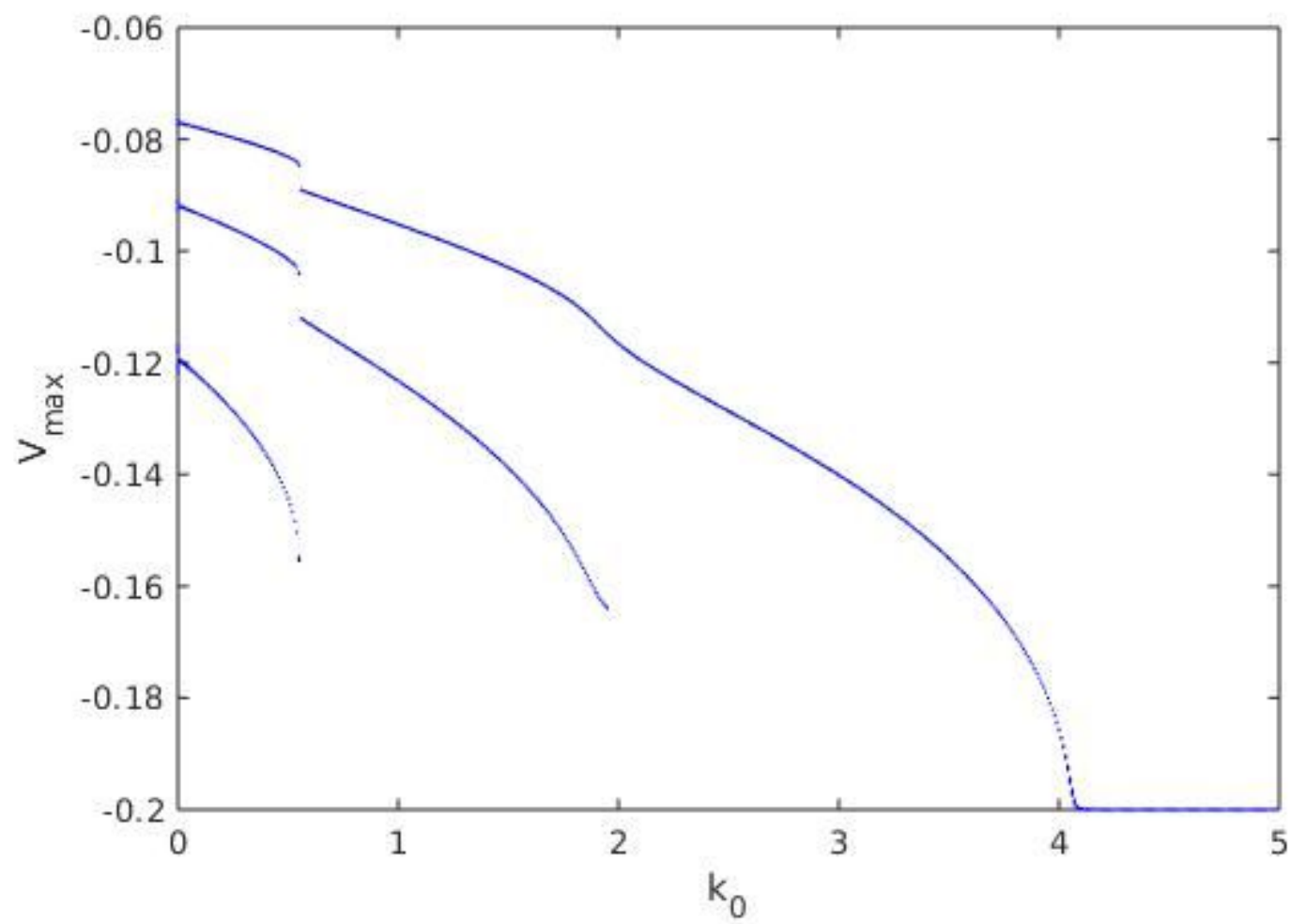

Figure 2

See manuscript for full figure caption.
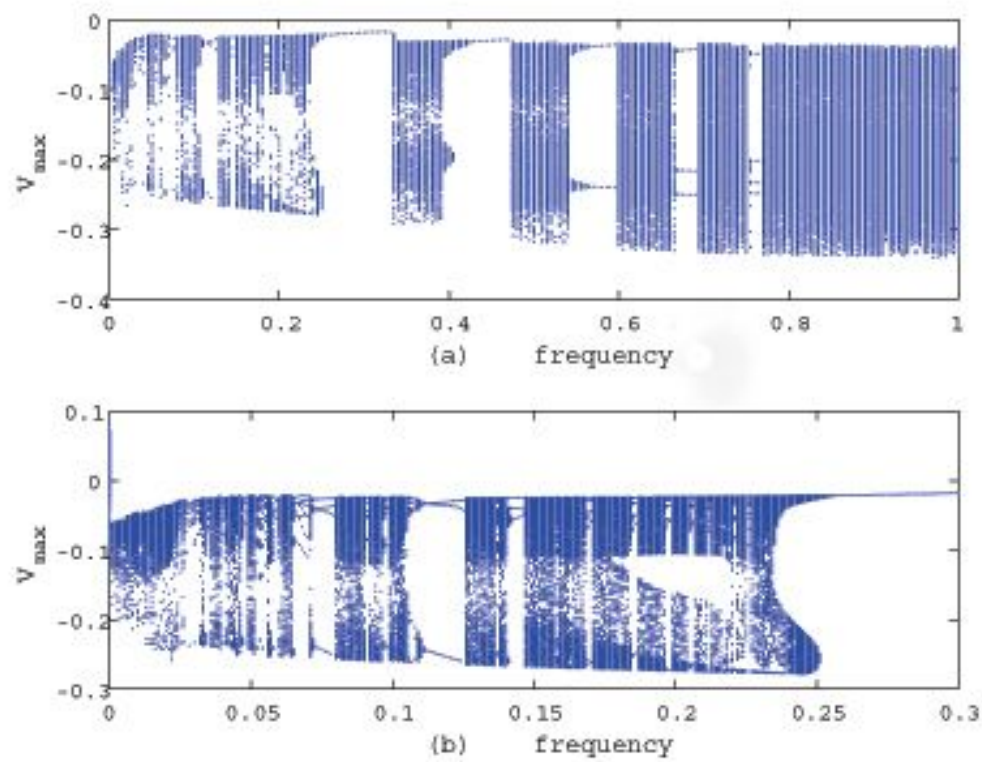

Figure 3

See manuscript for full figure caption. 

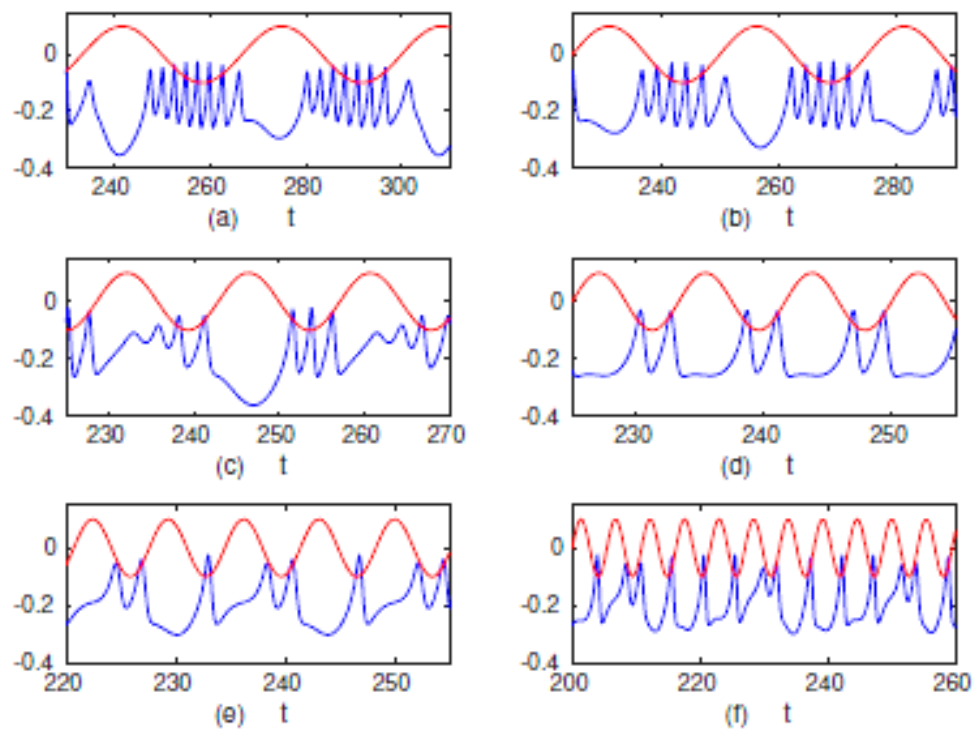

Figure 4

A selection of six frequency-locked states for (blue curves) for forcing amplitude, together with the forcing function (red curves). (a) a 16:2 frequency locked state; (b) a 12:2 locked state; (c) an 8:2 locked state; (d) a 2:1 locked state; (e ) a 3:2 locked state; (f) a 10:8 locked state.

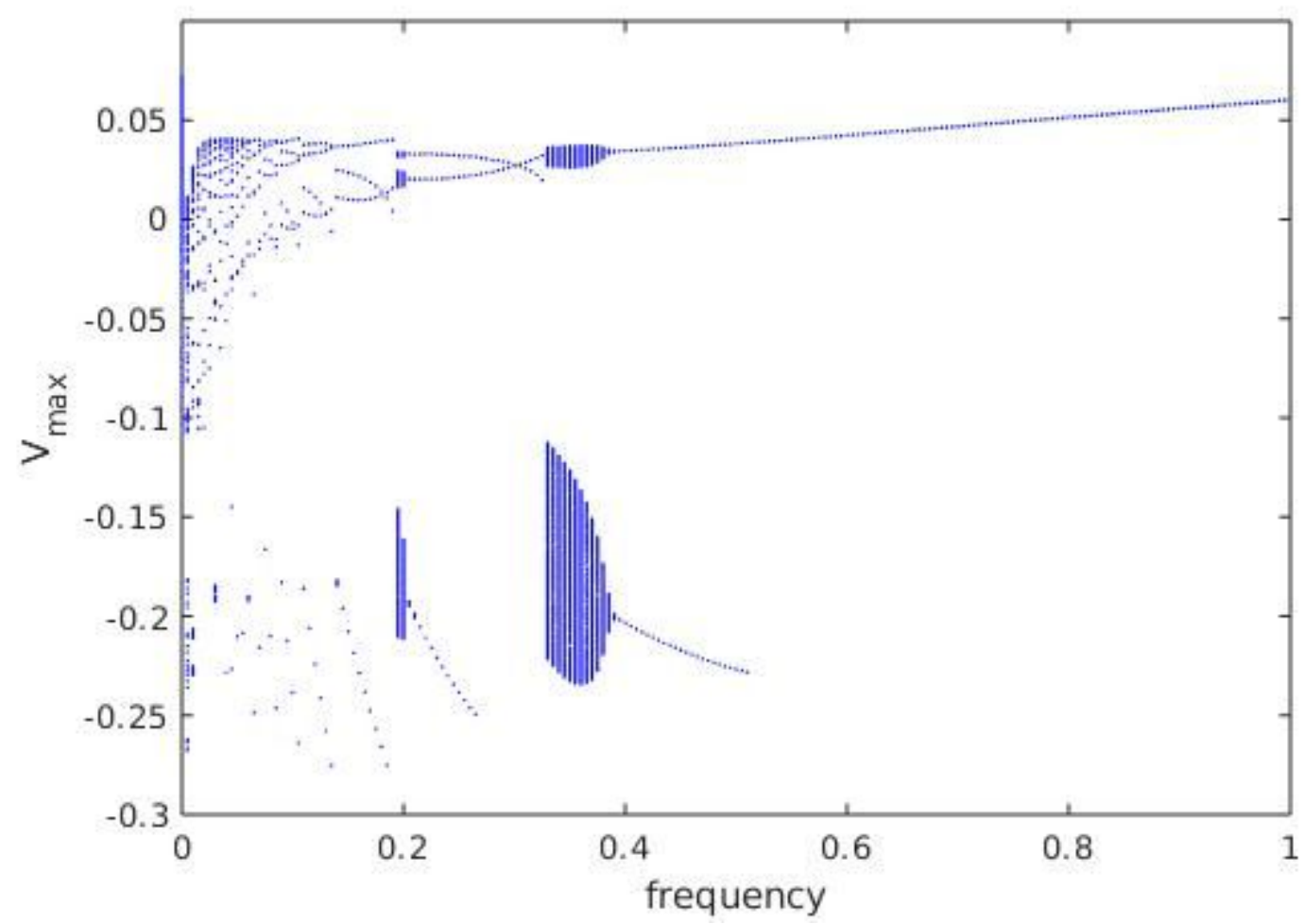

Figure 5

See manuscript for full figure caption. 

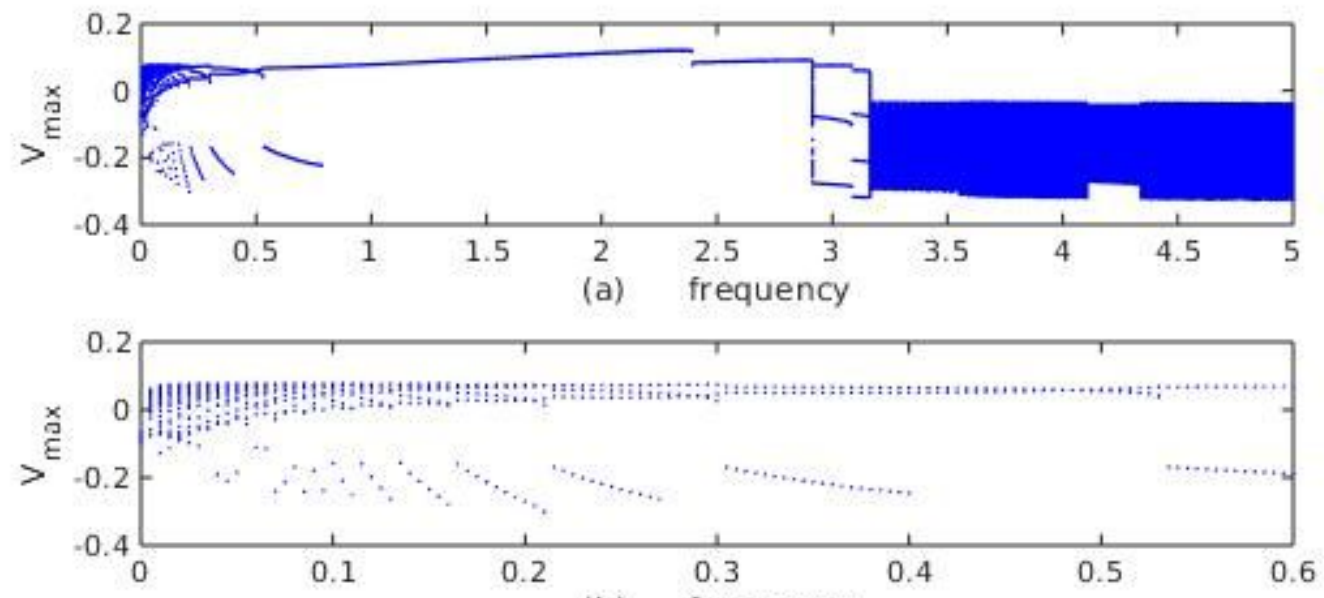

(b) frequency

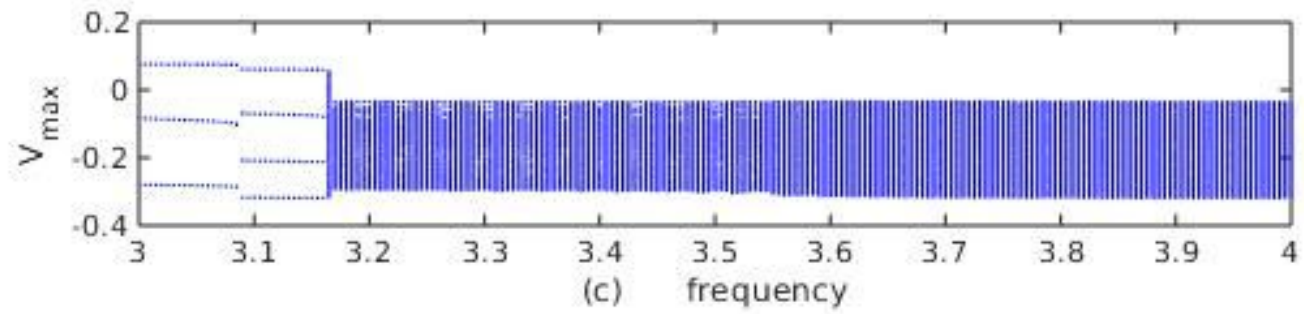

Figure 6

See manuscript for full figure caption.

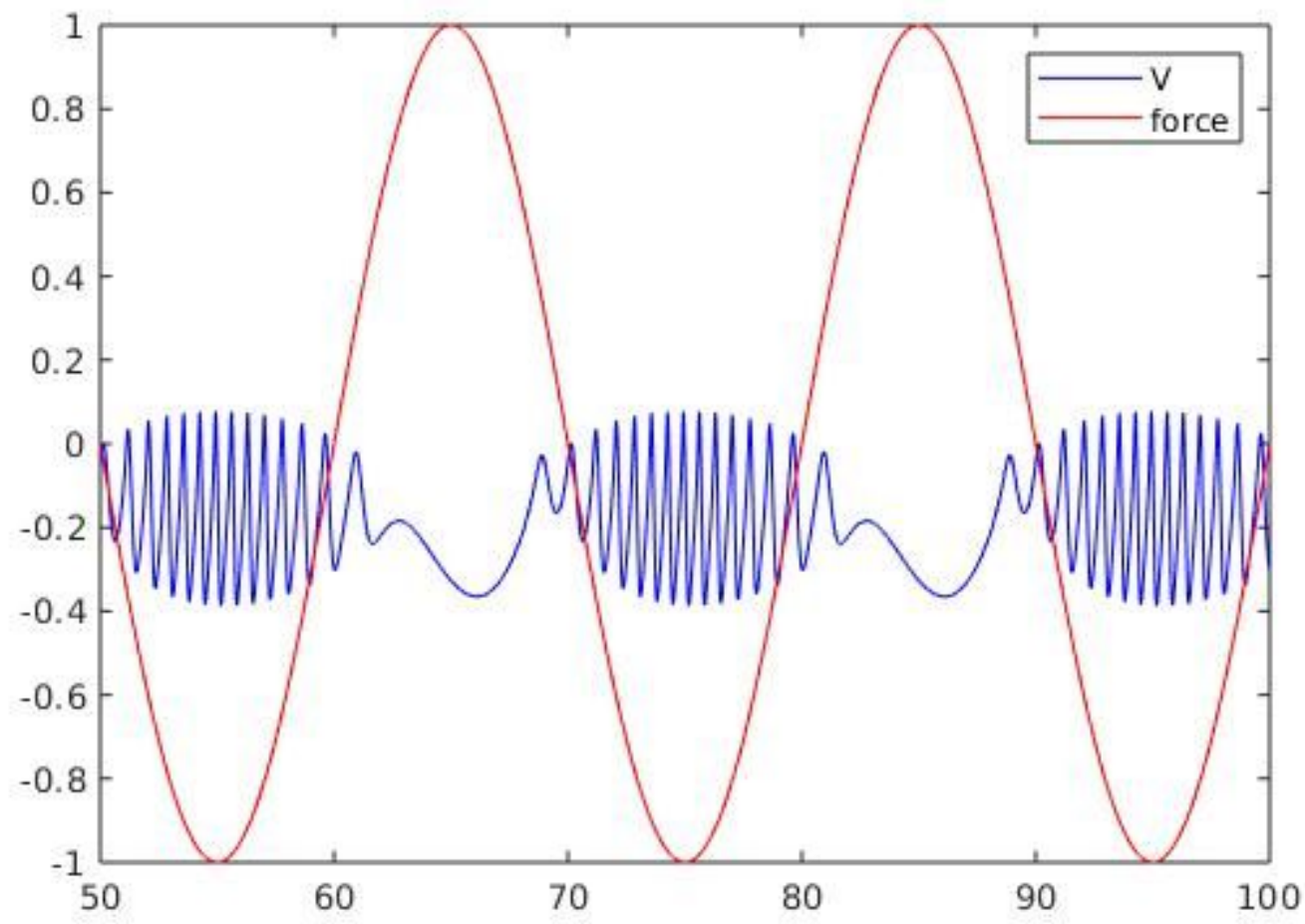

Figure 7 


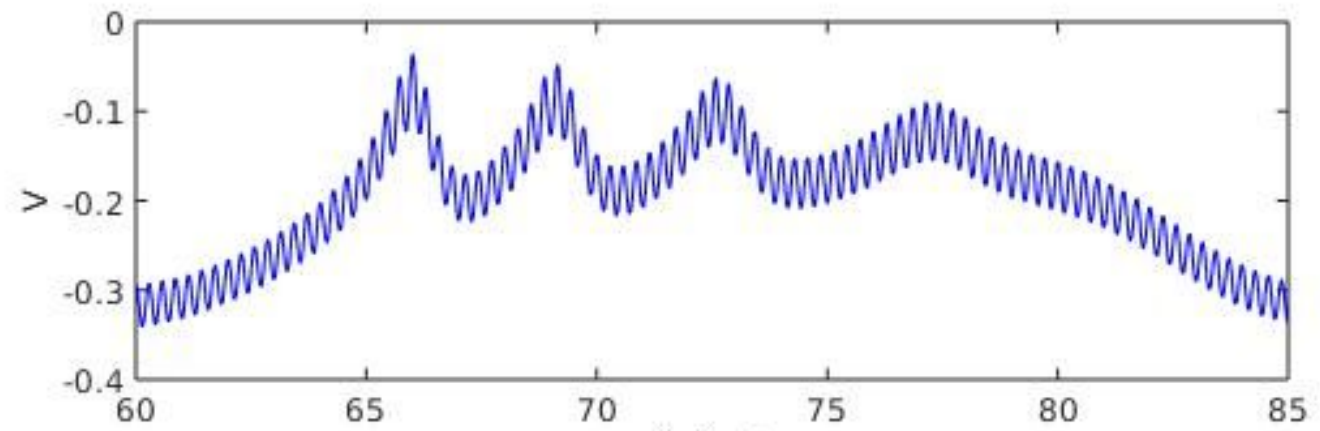

(a) $\mathrm{t}$

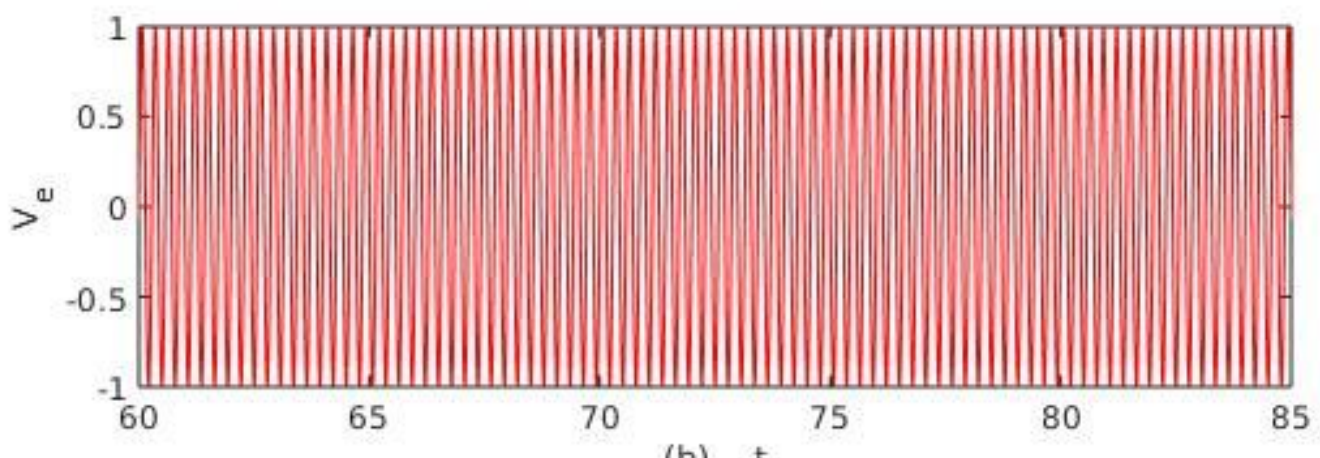

Figure 8

A section of the time series for $\mathrm{V}$, showing a quasi-periodic state for $1 \mathrm{E}=$ and frequency $3.5 \mathrm{w}=$. The upper panel shows $\mathrm{V}$, while the lower panel shows the forcing frequency.
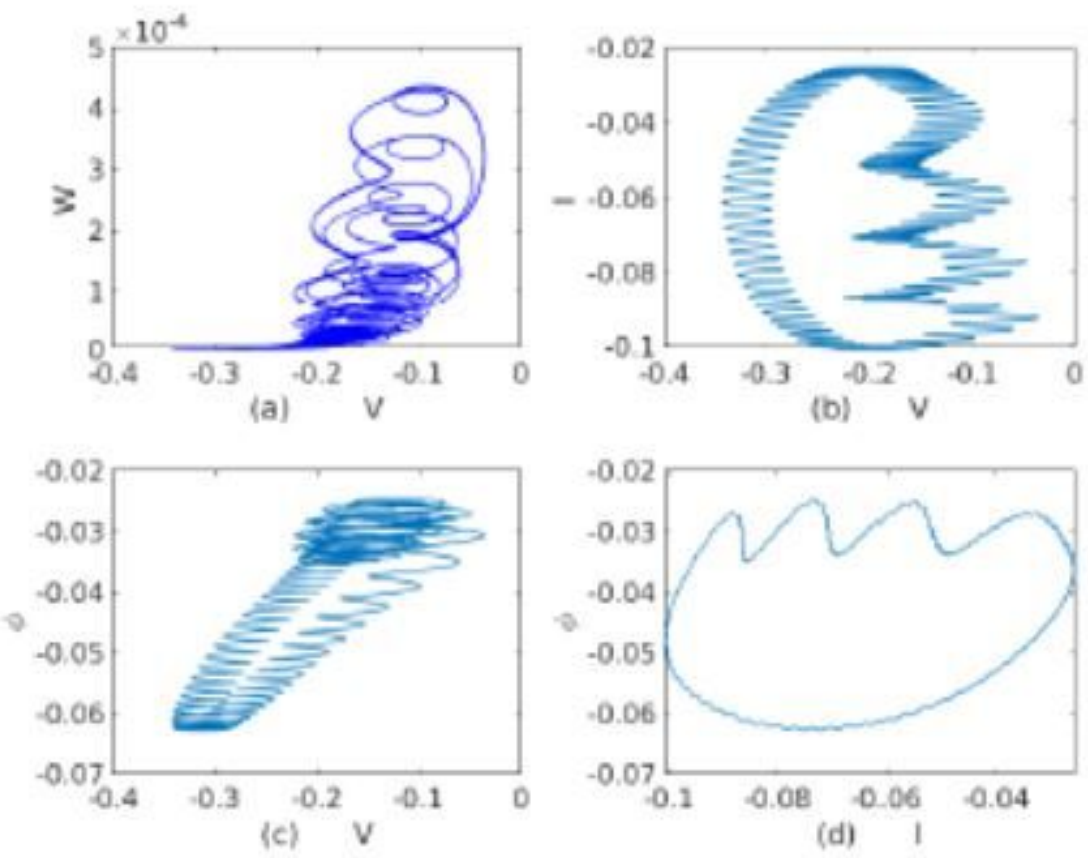

Figure 9 
Phase portraits for the quasi-periodic state for $1 \mathrm{E}=$ and frequency $3.5 \omega=$.

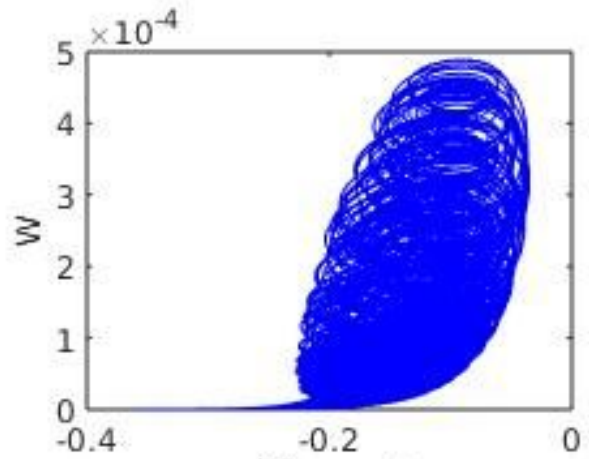

(a) $\mathrm{V}$

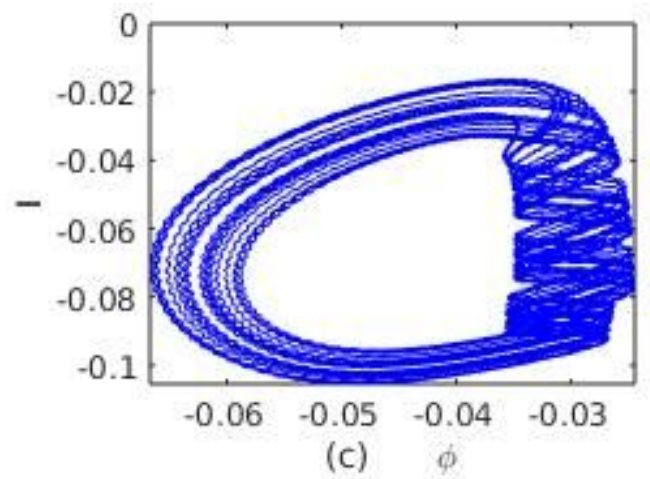

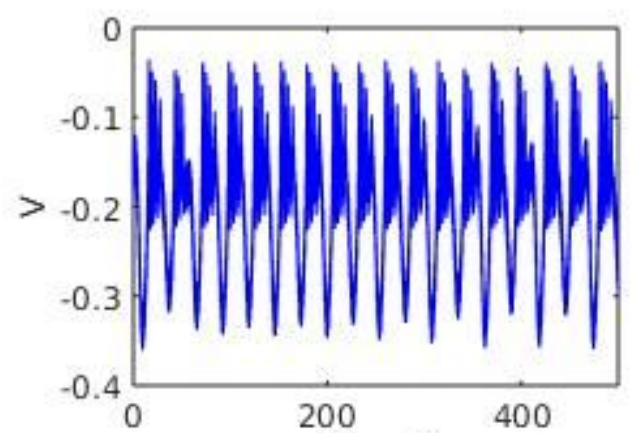

(b) t)

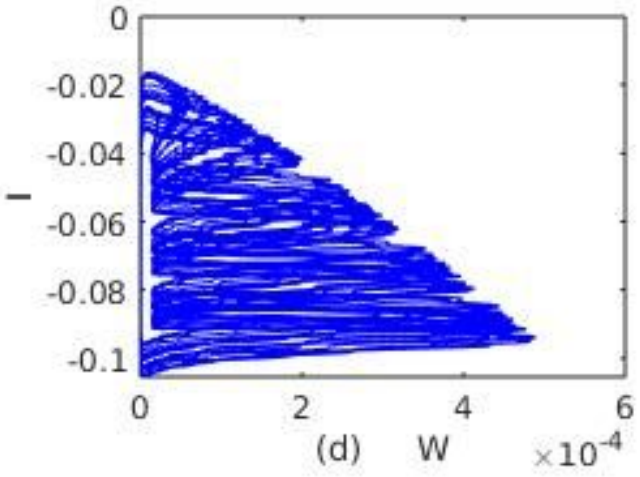

Figure 10

Phase portraits for the chaotic state for $1 \mathrm{E}=$ and frequency $4 \omega=$.
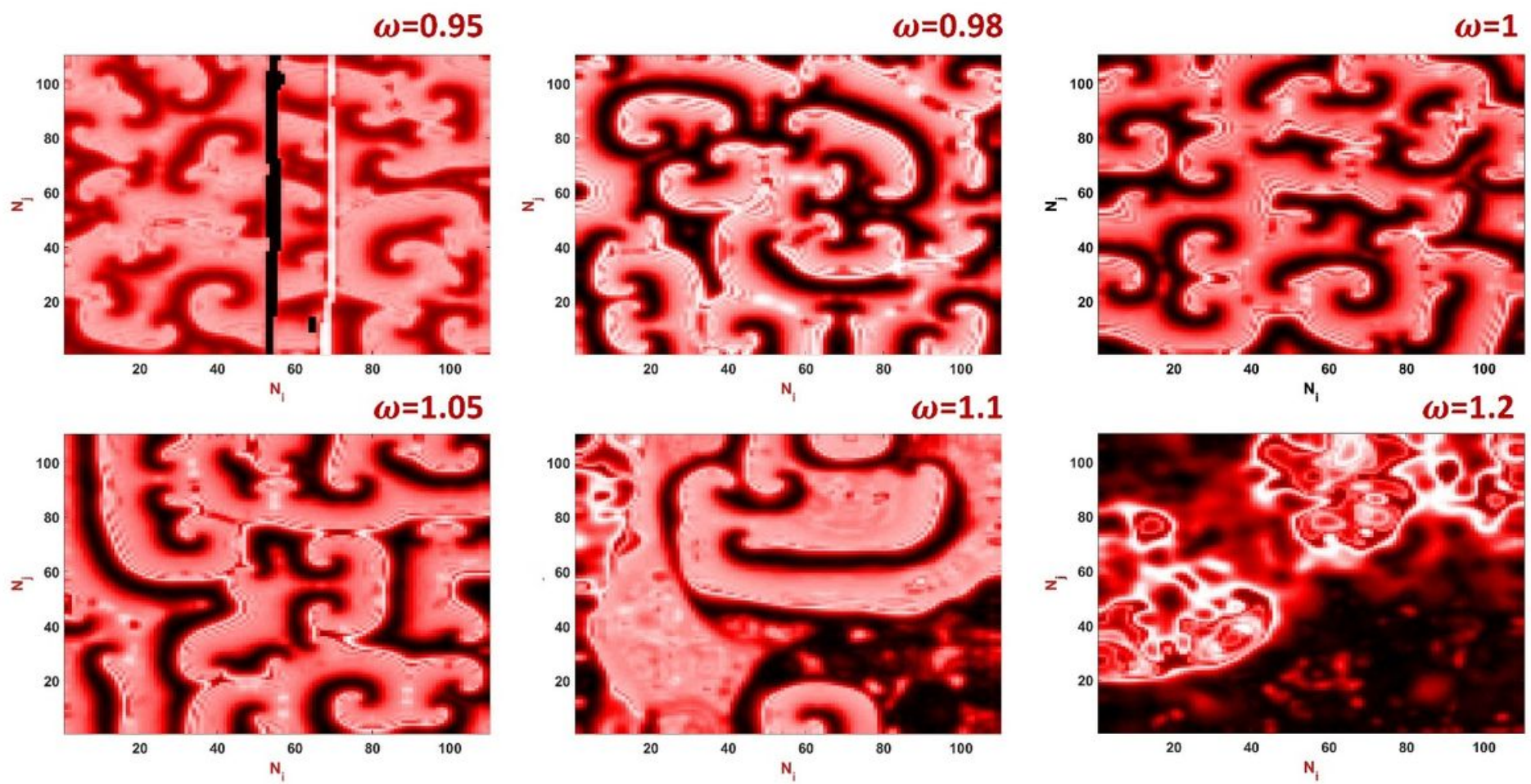

Figure 11 
Wave propagation in the network (4) considering the ML-EM neurons with electric field for different

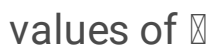
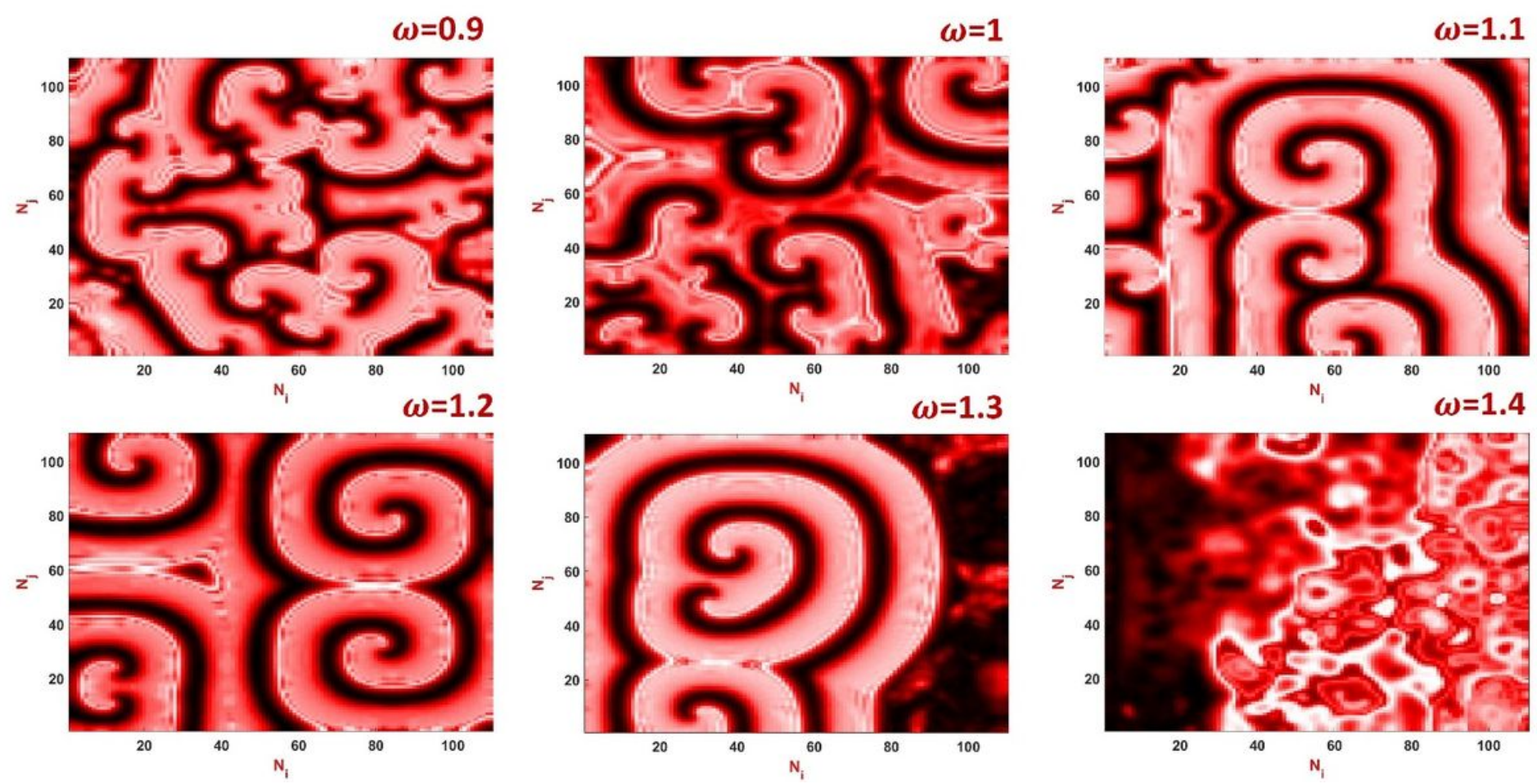

Figure 12

Wave propagation in the network (10) considering the ML-EM neurons with electric and magnetic fields for different values of $\otimes$

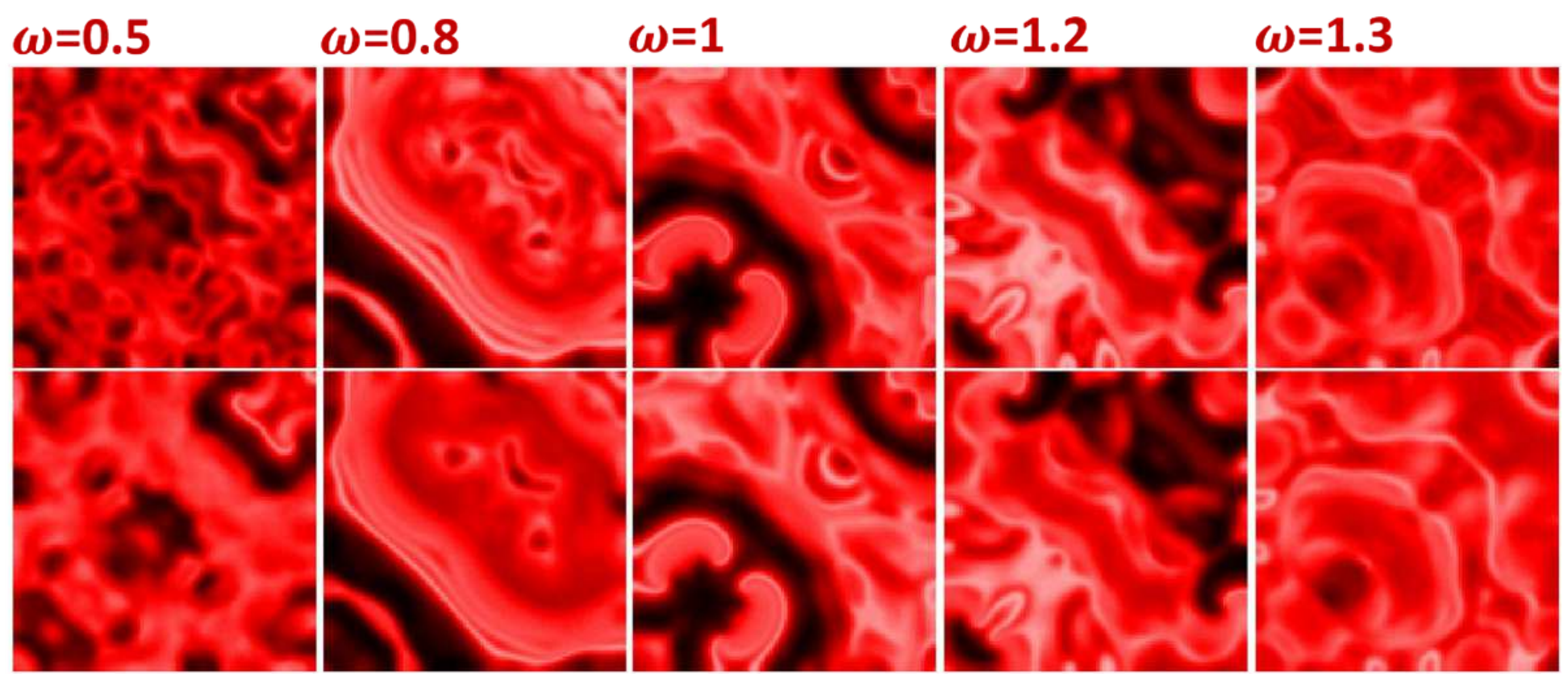

Figure 13 
Wave propagation in the two-layer network (5) considering the ML-EM neurons in the upper layer are exposed to electric field while the lower layer is considered to have no field effects. The snapshots are

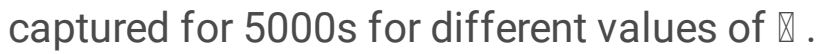

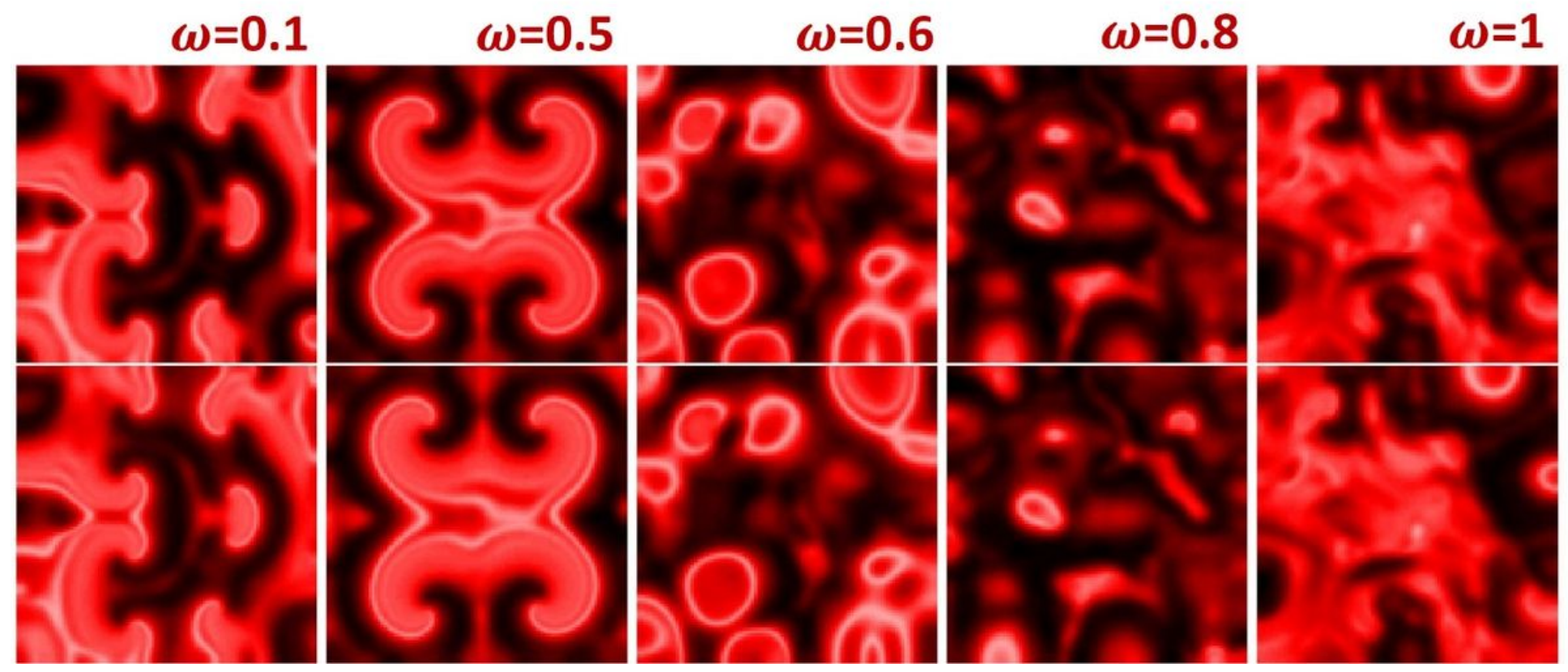

\section{Figure 14}

Wave propagation in the two-layer network (5) considering the ML-EM neurons in the upper layer are exposed to electric and magnetic fields while the lower layer is considered to have magnetic field effects. The snapshots are captured for 5000 s for different values of $\otimes$.

$\omega=0.1$
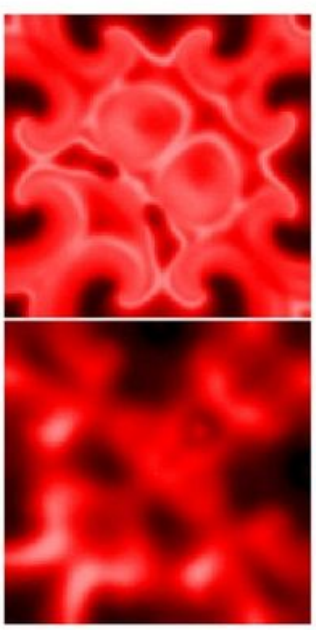

$\omega=0.5$

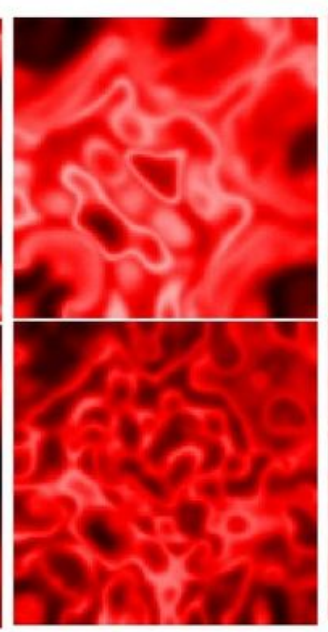

$\omega=1$

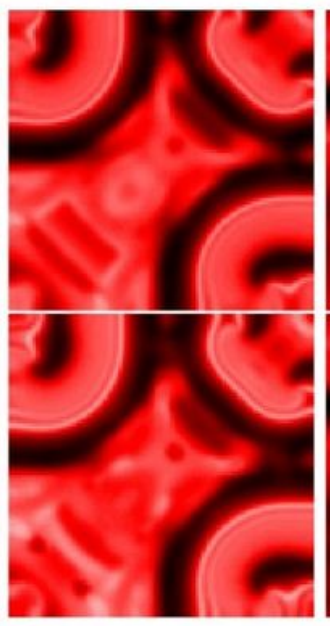

$\omega=1.5$

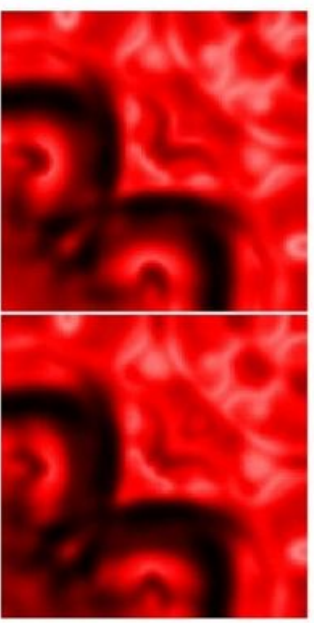

$\omega=1.8$

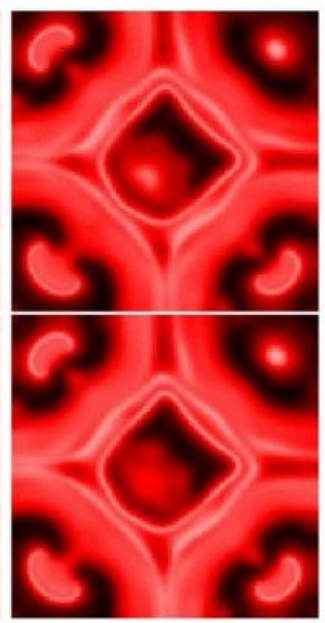

$\omega=2$

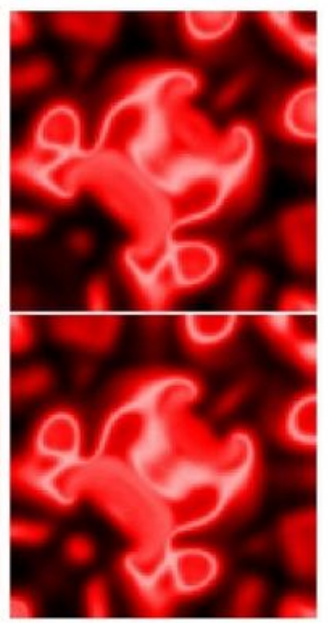

\section{Figure 15}

Wave propagation in the two-layer network (5) considering the ML-EM neurons in the lower layer are exposed to electric field while the upper layer is considered to have no field effects. The snapshots are

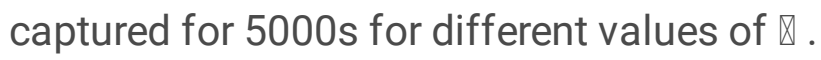


$\omega=0.1$

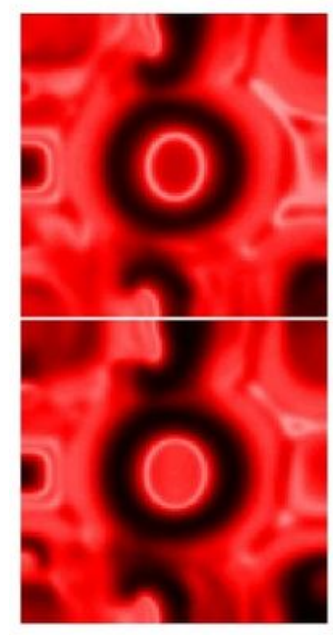

$$
\omega=1
$$

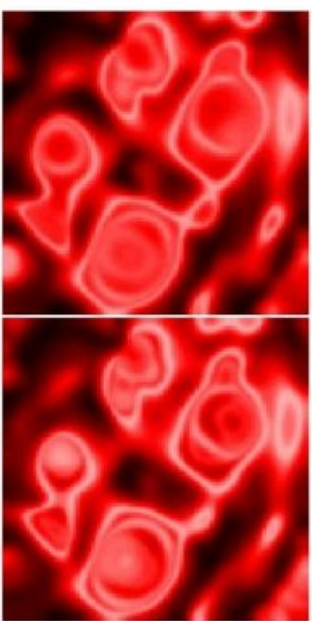

$\omega=3$

$\omega=5$

$\omega=7$

$\omega=12$
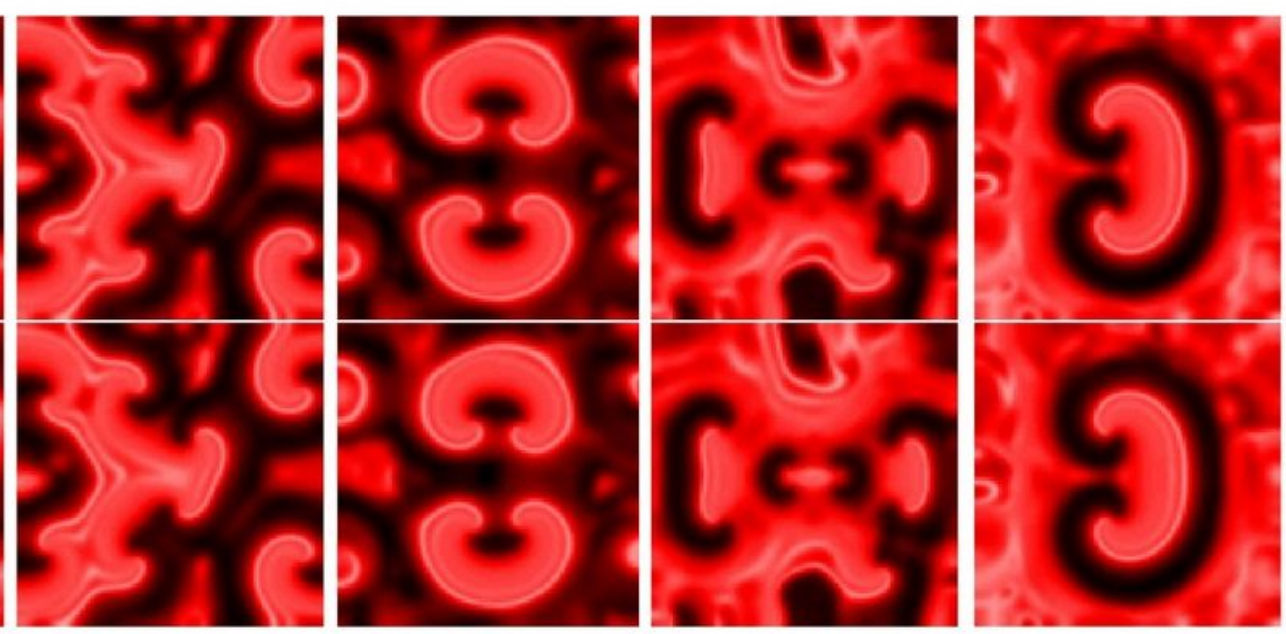

Figure 16

Wave propagation in the two-layer network (5) considering the ML-EM neurons in the lower layer are exposed to electric and magnetic fields while the upper layer is considered to have magnetic field effects. The snapshots are captured for 5000 s for different values of $\otimes$.
$\omega=0.5$
$\omega=0.8$
$\omega=1$
$\omega=1.2$
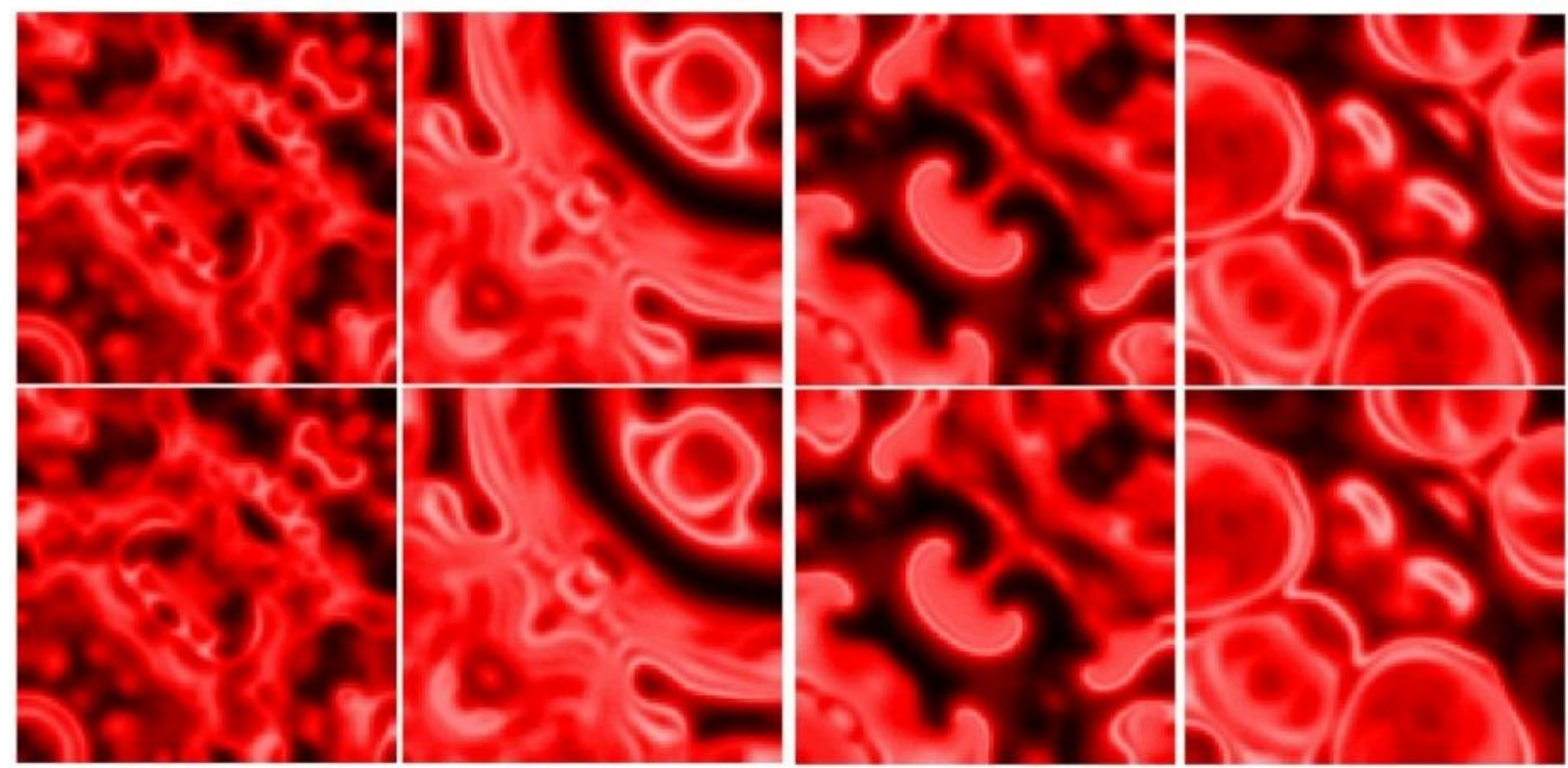

\section{Figure 17}

Wave propagation in the two-layer network (5) considering the ML-EM neurons in both the layers are

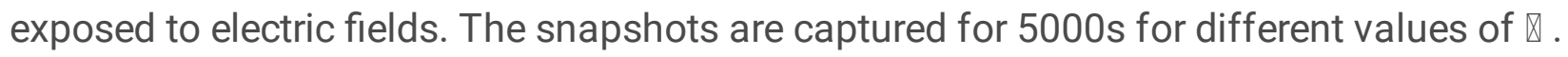



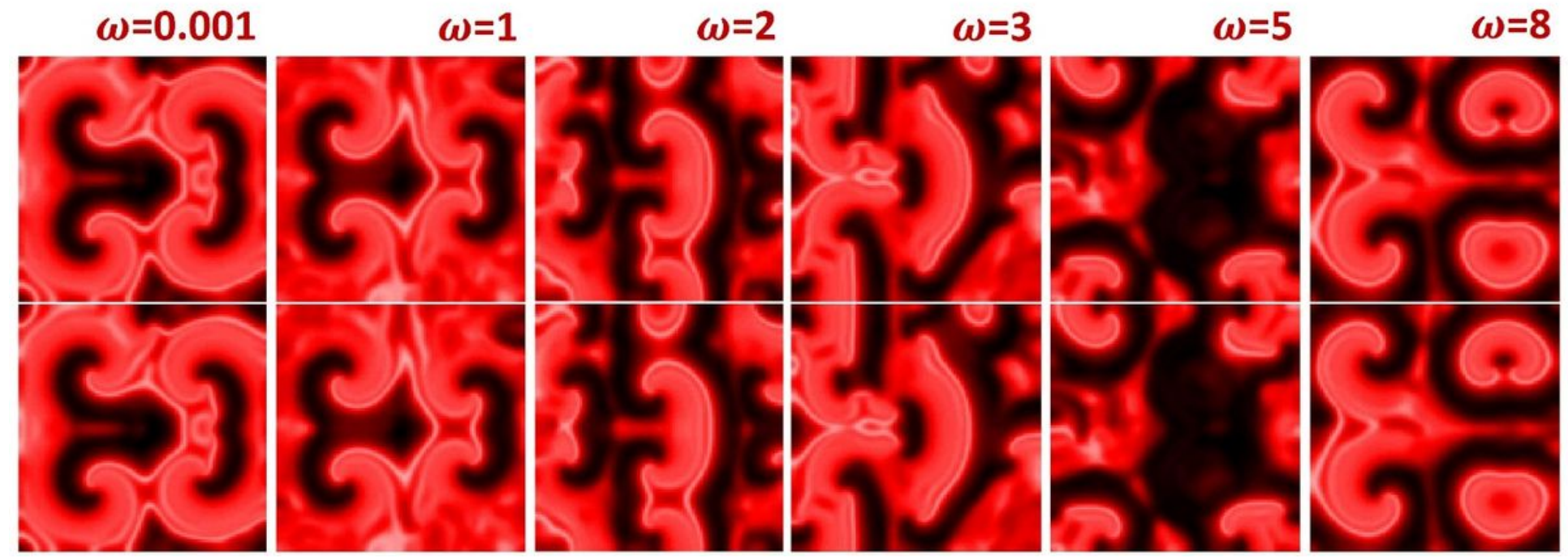

Figure 18

Wave propagation in the two-layer network (5) considering the ML-EM neurons in both the layers are

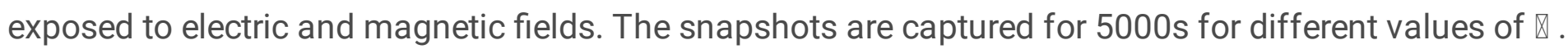

$$
\omega=0.5
$$$$
\omega=1
$$

$\omega=1.2$

$\omega=1.5$

$\omega=1.8$

$\omega=2$
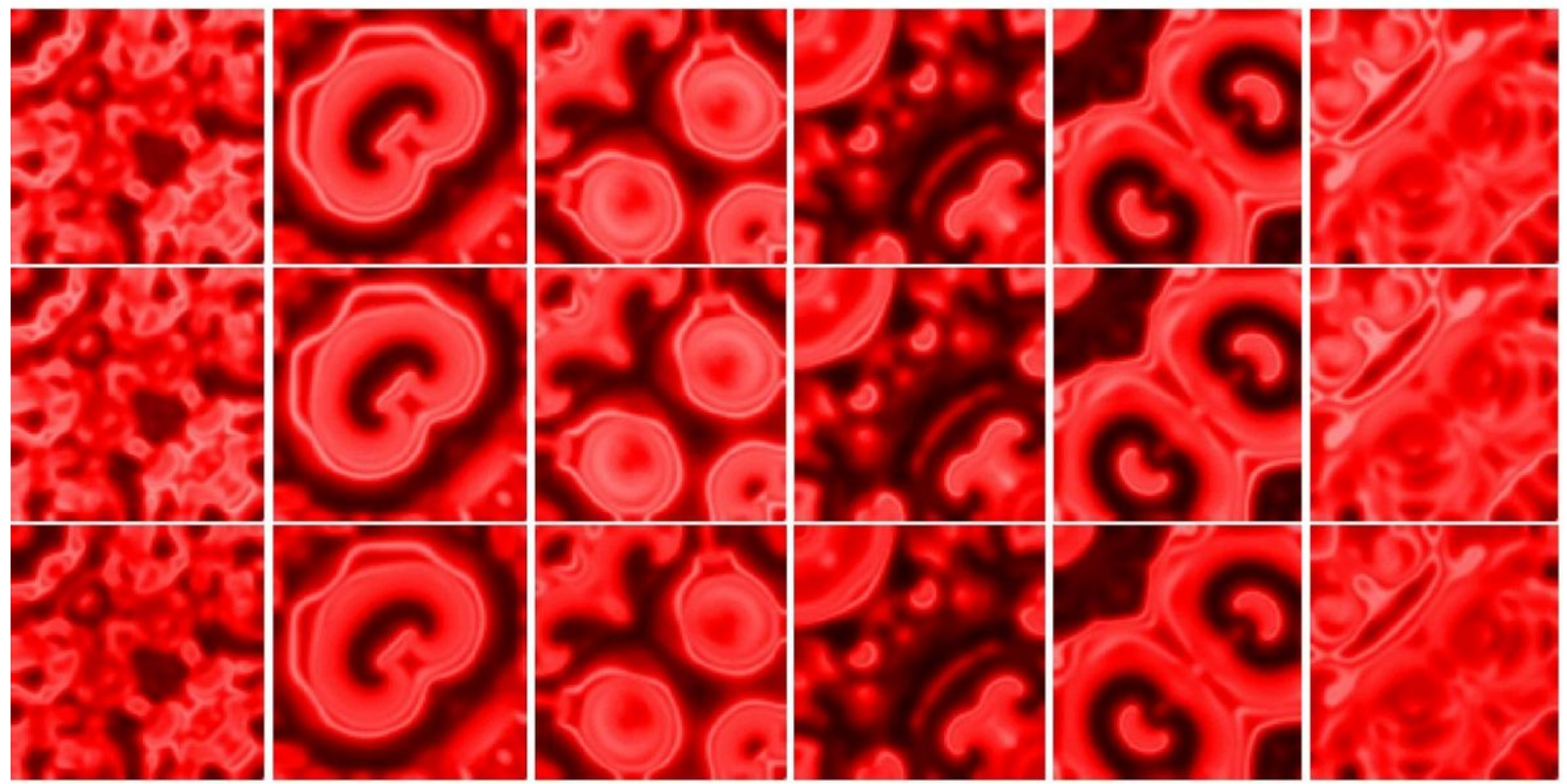

Figure 19

Wave propagation in the three-layer network (6) considering the ML-EM neurons in all the layers are exposed to electric fields. The snapshots are captured for 1000 s for different values of $\otimes$. 


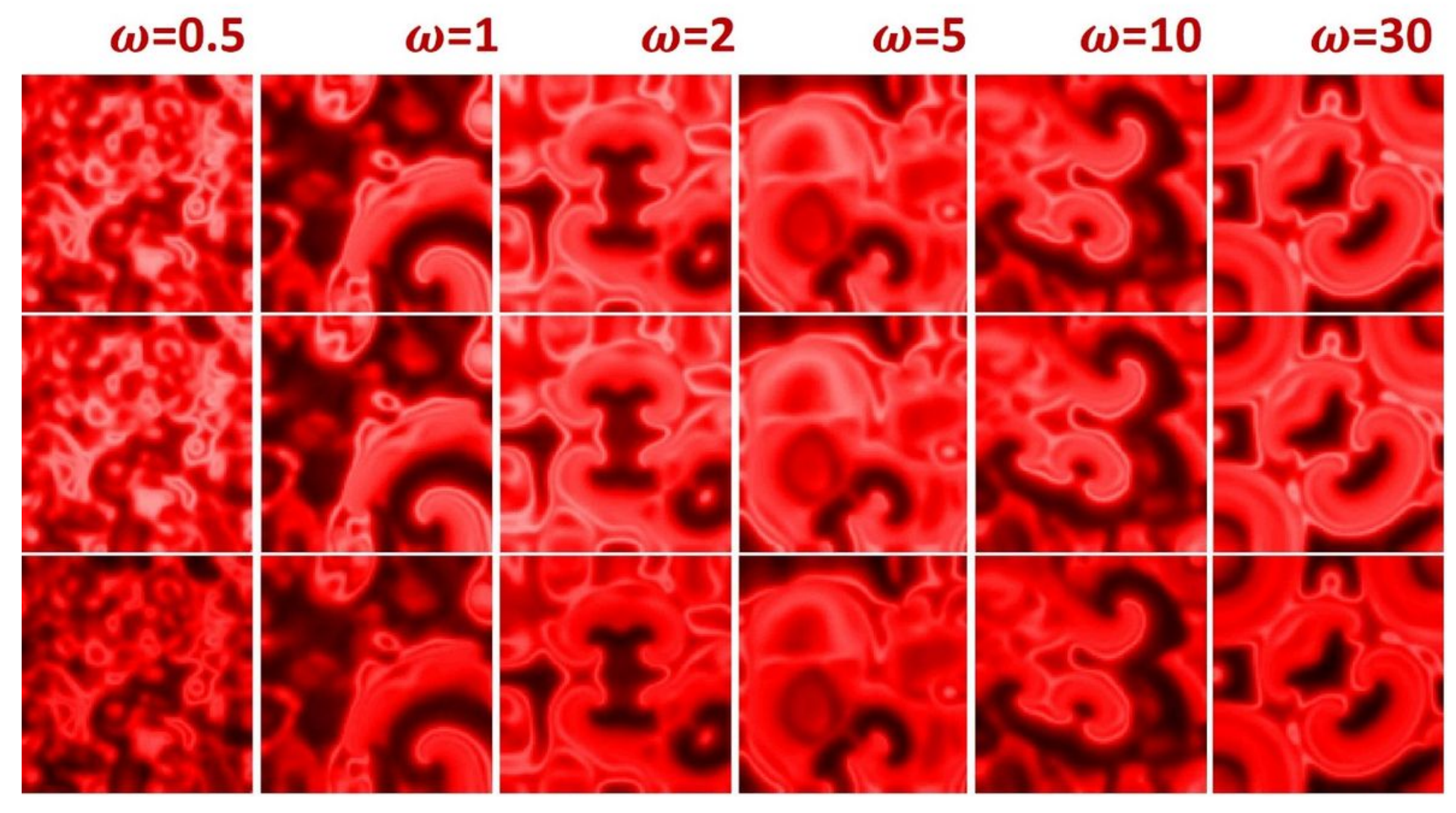

Figure 20

Wave propagation in the three-layer network (6) considering the ML-EM neurons in all the layers are exposed to electric and magnetic fields. The snapshots are captured for 1000 s for different values of $\omega$. 

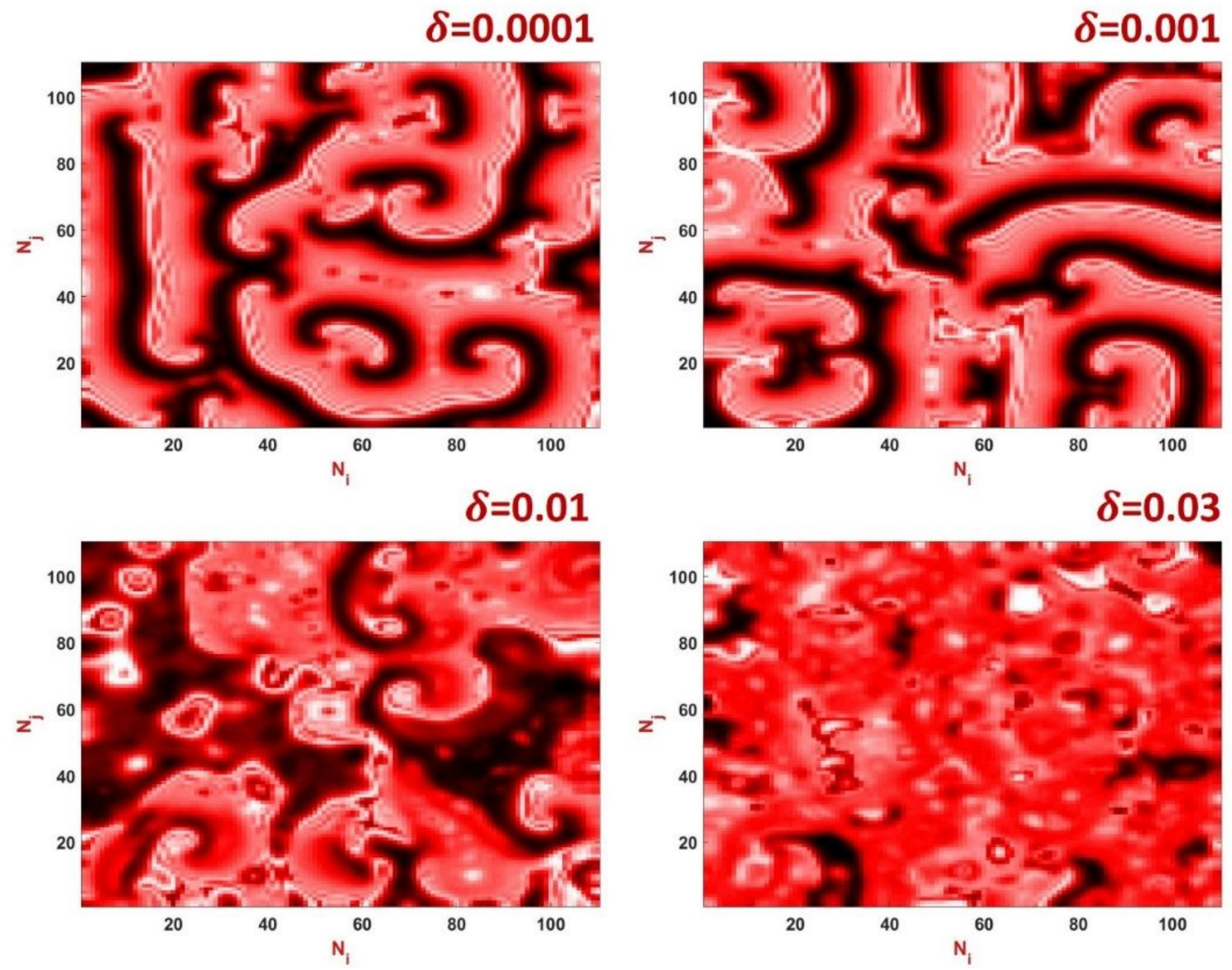

Figure 21

Effect of noise on the wave propagation in a single layer network. The noise variance $\delta$ is considered as the control parameter. 


\section{$\delta=0.0001$}

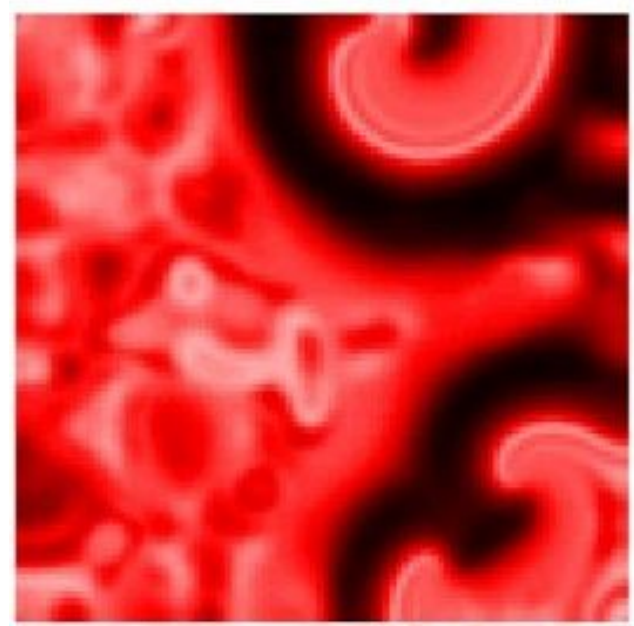

\section{$\delta=0.0002$}

$\delta=0.0003$
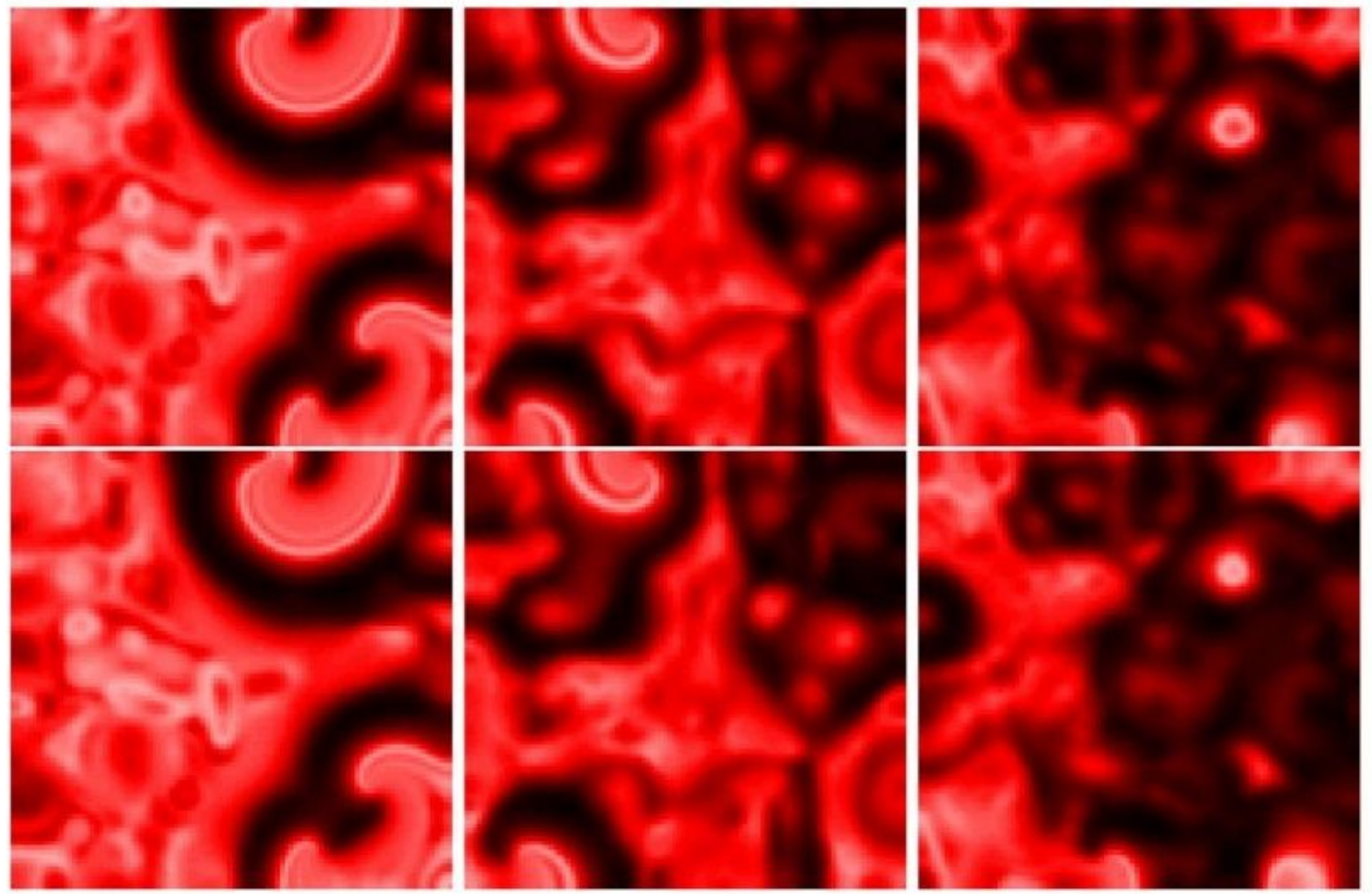

Figure 22

Effect of noise on the wave propagation in a two-layer network. The noise variance $\delta$ is considered as the control parameter. The electric field intensity is fixed as $\omega=1$. 


$$
\delta=0 \quad \delta=0.0001 \delta=0.0002 \delta=0.0005 \delta=0.0007
$$
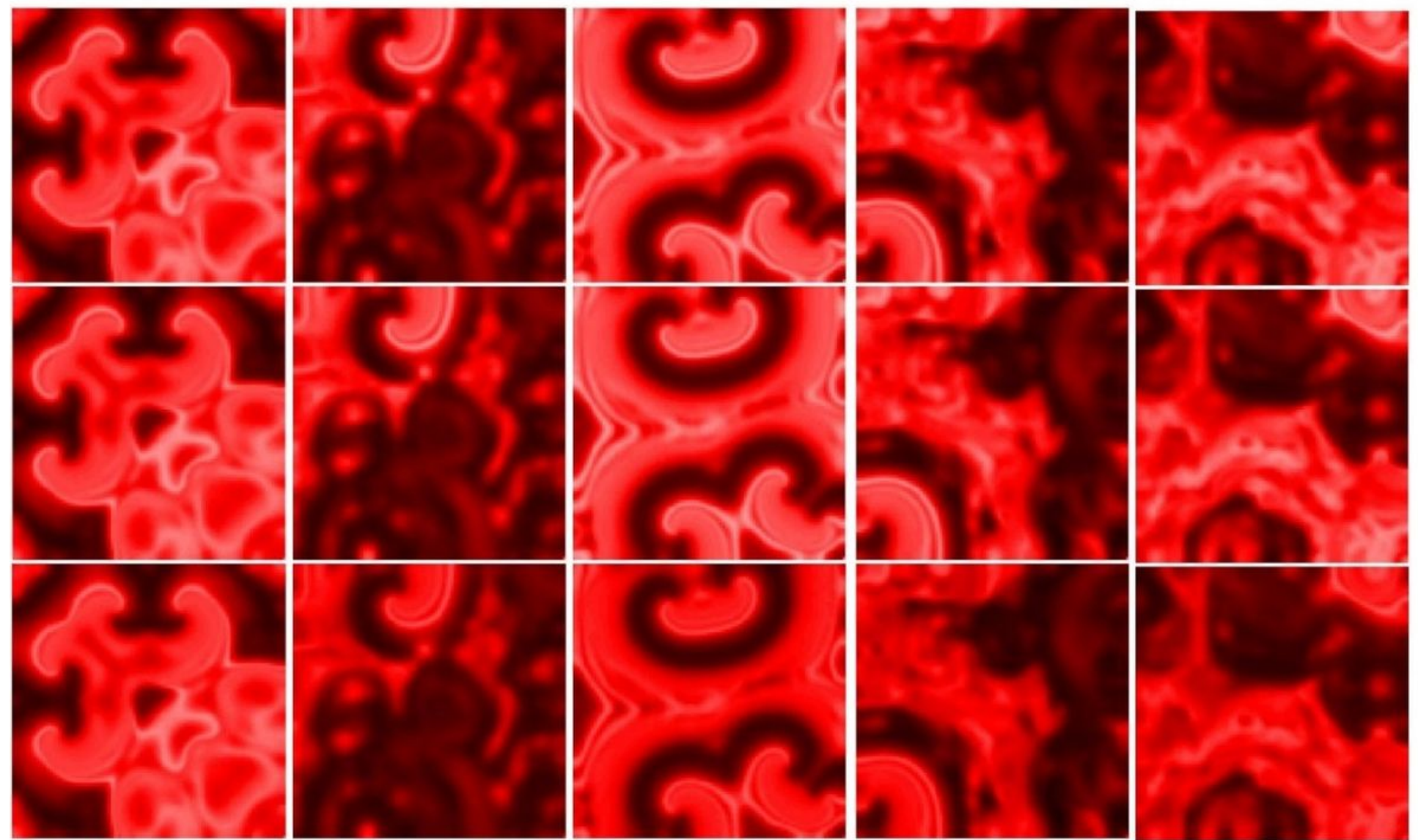

Figure 23

Effect of noise on the wave propagation in a two-layer network. The noise variance $\delta$ is considered as the control parameter. The electric field intensity is fixed as $\omega=1.2$. 